JOURNAL OF THE

AMERICAN MATHEMATICAL SOCIETY

Volume 16, Number 4, Pages 991-1032

S 0894-0347(03)00435-1

Article electronically published on July 8, 2003

\title{
THREE POINT COVERS WITH BAD REDUCTION
}

\author{
STEFAN WEWERS
}

\section{INTRODUCTION}

Ramified primes in the field of moduli. Let $f: Y \rightarrow X=\mathbb{P}_{\mathbb{C}}^{1}$ be a three point cover, i.e. a finite cover of the Riemann sphere branched at 0,1 and $\infty$. The 'obvious direction' of Belyi's theorem states that $f$ can be defined over a number field (see, e.g., [Koe]). Therefore, the absolute Galois group of $\mathbb{Q}$ acts on the set of isomorphism classes of all three point covers, and we can associate to $f$ the number field $K$ such that $\operatorname{Gal}(\overline{\mathbb{Q}} / K)$ is the stabilizer of the isomorphism class of $f$. The number field $K$ is called the field of moduli of $f$. Very little is known about the correspondence between three point covers and their associated field of moduli. One of the most general things that we know about $K$ is given by the following theorem of Beckmann [Bec89]. Let $G$ be the monodromy group of $f$. Then the extension $K / \mathbb{Q}$ is unramified outside the set of primes diving the order of $G$. This result is related to the fact that $f$ has good reduction at each prime ideal of $K$ dividing such primes $p$.

A recent theorem of Raynaud Ray99 gives a partial converse to Beckmann's theorem. Suppose that the prime $p$ strictly divides the order of $G$, i.e. that $p^{2}$ does not divide $|G|$. Let $\mathfrak{p}$ be a prime ideal of $K$ dividing $p$ and suppose that the ramification index $e(\mathfrak{p} / p)$ is strictly smaller than the number of conjugacy classes in $G$ of elements of order $p$. Then Raynaud's theorem asserts that $f$ has potentially good reduction at $\mathfrak{p}$.

The proof of this theorem depends on an analysis of the stable reduction of $f: Y \rightarrow X$ at $\mathfrak{p}$. If $f$ does not have potentially good reduction at $\mathfrak{p}$, then this analysis yields a certain lower bound for the ramification index $e(\mathfrak{p} / p)$. The theorem follows from this bound. We remark that this sort of result is not restricted to three point covers, but extends, under certain conditions, to covers of more general curves $X$. The essential condition here is that $p$ strictly divides the order of $G$.

In Wew03 the author has continued Raynaud's study of the stable reduction, and the present paper is a further continuation of this work. Our results yield an upper bound for the ramification index of $p$ in the field of moduli of certain three point covers. For instance, we prove the following theorem.

Theorem 1. Let $f: Y \rightarrow \mathbb{P}^{1}$ be a three point cover, with monodromy group $G$ and field of moduli $K$. Let $p$ be a prime which strictly divides the order of $G$. Then $p$ is at most tamely ramified in the extension $K / \mathbb{Q}$.

Received by the editors January 10, 2003.

2000 Mathematics Subject Classification. Primary 14H30, 11G20.

Key words and phrases. Three point cover, stable reduction, field of moduli. 
In fact we obtain a formula for the ramification index $e(\mathfrak{p} / p)$ in terms of the stable reduction of $f: Y \rightarrow X$ at $\mathfrak{p}$. This formula shows that $e(\mathfrak{p} / p)$ is prime to $p$ and gives an upper bound.

In contrast to the theorem of Raynaud mentioned above, our results are very particular to three point covers. They depend in an essential way on the fact that three point covers are 'rigid' objects, i.e. do not admit any nontrivial deformation.

Stable reduction. Let $R$ be a complete discrete valuation ring, with residue field $k$ of characteristic $p>0$ and fraction field $K$ of characteristic zero. Let $f_{K}: Y_{K} \rightarrow$ $X_{K}:=\mathbb{P}_{K}^{1}$ be a three point cover defined over $K$. By this we mean that $f_{K}$ is a finite cover between smooth and geometrically connected $K$-curves, ramified precisely at 0,1 and $\infty$. We assume moreover that $f_{K}$ is Galois, with Galois group $G$. In order to prove results such as Theorem 11 this is no restriction. After replacing the field $K$ by a finite extension, we may assume that $Y_{K}$ has a semistable model $Y_{R}$ over $R$ such that the ramification points of $f_{K}$ specialize to pairwise distinct smooth points on the special fiber. Among all semistable models with this property, we let $Y_{R}$ be the minimal one. The action of $G$ extends to $Y_{R}$; let $X_{R}:=Y_{R} / G$ denote the quotient. This is a semistable model of $\mathbb{P}_{K}^{1}$ over $R$. Therefore, the special fiber $\bar{X}:=X_{R} \otimes_{R} k$ is a tree of curves of genus zero. The map $f_{R}: Y_{R} \rightarrow X_{R}$ is called the stable model of the Galois cover $f_{K}$. Its restriction to the special fiber $\bar{f}: \bar{Y} \rightarrow \bar{X}$ is called the stable reduction of $f_{K}$.

We say that the cover $f_{K}: Y_{K} \rightarrow X_{K}$ has good reduction if the curves $X_{R}$ and $Y_{R}$ are smooth over $R$. If this is the case, then the stable reduction $\bar{f}: \bar{Y} \rightarrow \bar{X}=\mathbb{P}_{k}^{1}$ is a three point Galois cover in characteristic $p$. If $f_{K}$ does not have good reduction, we say that it has bad reduction. Our first main result is a structure theorem for the stable reduction $\bar{f}: \bar{Y} \rightarrow \bar{X}$ in the case of bad reduction.

Theorem 2. Suppose that $p$ strictly divides the order of $G$ and that the cover $f_{K}: Y_{K} \rightarrow X_{K}$ has bad reduction. Then the following hold (compare with Figure 11).

(1) The curve $\bar{X}$ consists of one central component $\bar{X}_{0}$, canonically isomorphic to $\mathbb{P}_{\underline{k}}^{1}$, and $r \geq 1$ tails $\bar{X}_{1}, \ldots, \bar{X}_{r}$, each of which intersects $\bar{X}_{0}$ in one point.

(2) Let $\bar{Y}_{0}$ be an irreducible component of $\bar{Y}$ lying above the central component $\bar{X}_{0}$. Then the map $\bar{Y}_{0} \rightarrow \bar{X}_{0}$ induced from $\bar{f}$ is the composition of a purely inseparable map $\bar{Y}_{0} \rightarrow \bar{Z}_{0}$ of degree $p$ and a Galois cover $\bar{Z}_{0} \rightarrow \bar{X}_{0}$. The Galois group of $\bar{Z}_{0} \rightarrow \bar{X}_{0}$ is isomorphic to $N / I$, where $N$ is the stabilizer of the component $\bar{Y}_{0}$ and $I \cong \mathbb{Z} / p$ is its inertia group. The cover $\bar{Z}_{0} \rightarrow \bar{X}_{0}$ is ramified at most at the points where $\bar{X}_{0}$ meets one of the tails $\bar{X}_{j}$.

(3) For $j=1, \ldots, r$, let $\bar{Y}_{j}$ be an irreducible component of $\bar{Y}$ lying over the tail $\bar{X}_{j}$. Then the map $\bar{Y}_{j} \rightarrow \bar{X}_{j}$ induced by $\bar{f}$ is a Galois cover, ramified at most at two points. More precisely, $\bar{Y}_{j} \rightarrow \bar{X}_{j}$ is wildly ramified at the point where $\bar{X}_{j}$ intersects $\bar{X}_{0}$ and is tamely ramified at any point which is the specialization of one of the $K$-points 0,1 or $\infty$.

Actually, parts (2) and (3) follow from part (1), by the results of [Ray99. Moreover, this implication is still valid if we allow more than three branch points or replace $X_{K}=\mathbb{P}_{K}^{1}$ by any smooth proper $K$-curve with good reduction (provided that the branch points do not coalesce on the special fiber). On the other hand, the assumption that $f_{K}$ is a three point cover is crucial for part (1) to hold. 


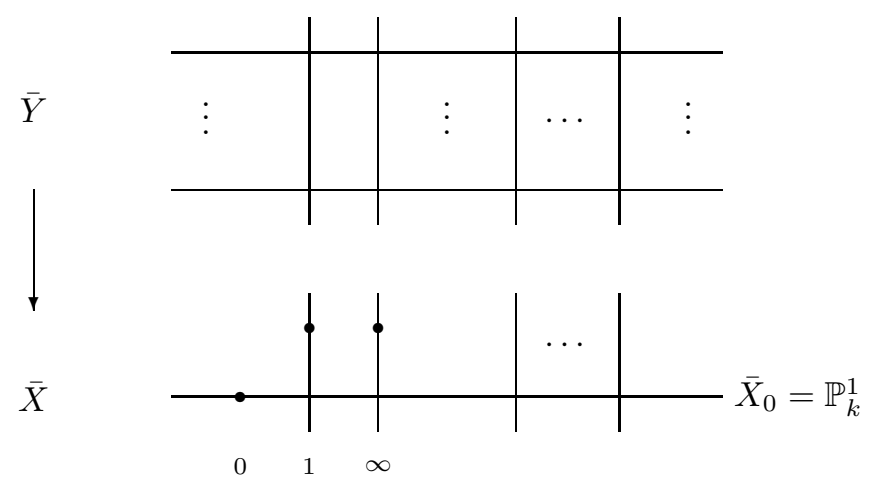

FiguRE 1. The stable reduction of a three point cover

Under the additional assumption that all the ramification indices of $f_{K}$ are prime to $p$, Theorem 2 (1) can be deduced from the results of Wew03, via Raynaud's construction of the auxiliary cover (see the introduction of [Wew03]). The proof of the general case given in the present paper is based on a generalization of the methods of [Wew03]. This generalization avoids the assumption on the ramification indices and the use of the auxiliary cover.

Lifting of special $G$-maps. The stable reduction of a three point Galois cover $f_{K}: Y_{K} \rightarrow X_{K}:=\mathbb{P}_{K}^{1}$ is, by definition, a finite map $\bar{f}: \bar{Y} \rightarrow \bar{X}$ between semistable curves over the residue field $k$, together with an action of a finite group $G$ on $\bar{Y}$ which commutes with $\bar{f}$. In the case of bad reduction, the curves $\bar{X}$ and $\bar{Y}$ are both singular, and the map $\bar{f}$ is inseparable over some of the irreducible components of $\bar{X}$. This suggests the following question. Given a map $\bar{f}: \bar{Y} \rightarrow \bar{X}$ of the sort we have just described, does it occur as the stable reduction of a three point Galois cover $f_{K}: Y_{K} \rightarrow X_{K}$ in characteristic zero? If this is the case, then we say that $f_{K}: Y_{K} \rightarrow X_{K}$ is a lift of $\bar{f}: \bar{Y} \rightarrow \bar{X}$.

Theorem 2 gives a list of necessary conditions on $\bar{f}$ for the existence of a lift (under the condition that $p$ strictly divides the order of the group $G$ ). There is one extra condition on $\bar{f}$ which we have omitted from the statement of Theorem 2 but which plays a central role in this paper. This additional condition is the existence of a certain differential form $\omega_{0}$ on the curve $\bar{Z}_{0}$ which is 'compatible' with $\bar{f}$ (see Theorem 2 (2) for the definition of $\bar{Z}_{0}$ ). For instance, $\omega_{0}$ is logarithmic, i.e. of the form $\mathrm{d} u / u$, and it is an eigenvector under the action of the Galois group of the cover $\bar{Z}_{0} \rightarrow \bar{X}_{0}$. Moreover, the divisor of zeroes and poles of $\omega_{0}$ is related to and determined by the map $\bar{f}$ and the action of $G$ on $\bar{Y}$. The existence and the basic properties of $\omega_{0}$ follow from work of Green-Matignon [GM99] and Henrio Hen00. In some sense, $\omega_{0}$ carries infinitesimal information about the action of $G$ on $\bar{Y}$ which 'got lost' by reduction to characteristic $p$. Following Wew03, we call $\left(\bar{Z}_{0}, \omega_{0}\right)$ a special deformation datum. (Actually, in [Wew03] we assume that $\omega_{0}$ has no poles. This is true if and only if all the ramification indices of the three point cover $f_{K}: Y_{K} \rightarrow X_{K}$ are prime to $p$.) 
In the present paper we define the notion of a special $G$-map. This is a map $\bar{f}: \bar{Y} \rightarrow \bar{X}$ between semistable curves in characteristic $p$ together with an action of a finite group $G$ on $\bar{Y}$ satisfying certain conditions. Essentially, these conditions say that $\bar{f}$ satisfies the conclusion of Theorem 2 and that there exists a special deformation datum $\left(\bar{Z}_{0}, \omega\right)$ which is 'compatible' with $\bar{f}: \bar{Y} \rightarrow \bar{X}$ and the action of $G$ on $\bar{Y}$. Our second main result can be stated as follows.

Theorem 3. Let $\bar{f}: \bar{Y} \rightarrow \bar{X}$ be a special G-map, defined over an algebraically closed field $k$ of characteristic $p>0$. Let $K_{0}$ denote the fraction field of the ring of Witt vectors over $k$. Then the following hold.

(1) There exists a lift of $\bar{f}$, i.e. a three point Galois cover $f_{K}: Y_{K} \rightarrow X_{K}$ whose stable reduction is isomorphic to $\bar{f}: \bar{Y} \rightarrow \bar{X}$.

(2) Each lift of $\bar{f}: \bar{Y} \rightarrow \bar{X}$ can be defined over a finite extension $K / K_{0}$ which is at most tamely ramified.

We actually prove more. Namely, we determine the set of isomorphism classes of all lifts of $\bar{f}$, together with the action of $\operatorname{Gal}\left(\bar{K}_{0} / K_{0}\right)$ on this set, in terms of certain numerical invariants attached to $\bar{f}$. From this more precise result one can deduce an upper bound for the degree of the minimal extension $K / K_{0}$ over which a given lift of $\bar{f}$ can be defined. In any case, Theorem 1 follows from Theorem 2 and Theorem 3 by a straightforward argument.

Part (1) of Theorem 3 i.e. the mere existence of a lift, follows already from the results of Wew03] (assuming that $\omega_{0}$ has no poles). Part (2) is more difficult and needs new methods. The key step in the proof of (2) is the study of the deformation theory of a certain curve with group scheme action associated to the special deformation datum $\left(\bar{Z}_{0}, \omega_{0}\right)$. A detailed exposition of this deformation theory can be found in Wew02.

This paper is divided into four sections. The first section contains the general theory of stable reduction of Galois covers, the monodromy action and deformation data. In $\$ 2$ we apply this theory to the case of three point covers and prove our first main result, which corresponds to Theorem 2 above. We also define the notion of a special $G$-map. In $\$ 3$ we apply the deformation theory of Wew02 in order to lift a special deformation datum to an auxiliary cover with certain good properties. This is preparatory work for $\$ 4$, where we prove the lifting result corresponding to Theorem 3. At the end of 4 we draw some conclusions from this result, concerning the field of moduli. In particular, we prove Theorem 3 . We also discuss in some detail the case of three point covers of genus 0 .

Acknowledgments. This work grew out of discussions with Michel Raynaud on my previous paper [Wew03. I thank him heartily for his critical remarks and his encouragement. The final version of this paper was written while I was a guest of the Max-Planck-Institut in Bonn.

\section{The stable reduction of Galois covers}

Let $K$ be a field of characteristic zero equipped with a complete discrete valuation whose residue field $k$ is algebraically closed and has characteristic $p>0$. Let $f: Y \rightarrow X$ be a Galois cover of smooth projective curves over $K$. In this section we define and study the stable reduction of $f$. We make two crucial assumptions on the reduction of $f$. First, we assume that the marked curve $(X, S)$ (where $S$ 
is the branch locus of $f$ ) has good reduction. Second, we assume that $f$ has mild reduction, a property which is automatically satisfied if $p$ strictly divides the order of the Galois group of $f$.

We start by recalling some results of Raynaud Ray99] on the structure of $\bar{f}$ : $\bar{Y} \rightarrow \bar{X}$. Then we attach to each irreducible component of $\bar{X}$ where the stable reduction $\bar{f}: \bar{Y} \rightarrow \bar{X}$ is inseparable a so-called deformation datum. This datum encodes infinitesimal information on the action of the Galois group of $f: Y \rightarrow X$. The deformation data attached to the individual components of $\bar{X}$ are related by certain compatibility conditions. The existence of a set of compatible deformation data imposes strong restrictions on the map $\bar{f}: \bar{Y} \rightarrow \bar{X}$. In this section, we content ourselves with working out those restrictions which are of a purely combinatorial nature. For instance, we obtain a new proof of Raynaud's vanishing cycle formula Ray99.

Most of the results of this section are already present in [Wew03, in a special case.

\subsection{The stable model and the monodromy action.}

1.1.1. The following notation is fixed throughout $\$ 1$ and 92 . Let $R_{0}$ be a complete discrete valuation ring, with quotient field $K_{0}$ of characteristic 0 and residue field $k$ of characteristic $p>0$. We assume that $k$ is algebraically closed. We fix an algebraic closure $\bar{K}$ of $K_{0}$.

Let $\left(X_{0}, S_{0}\right)$ be a smooth, stably marked curve over $R_{0}$ (see [Knu83 for the definition of stably marked curves). Since $R_{0}$ is strictly henselian, the smoothness of $X_{0}$ implies that $S_{0}=\left\{x_{R_{0}, j} \mid j \in B_{0}\right\}$, where $x_{R_{0}, j}$ : Spec $R_{0} \rightarrow X_{0}$ are pairwise distinct sections, indexed by a finite set $B_{0}$. If $K / K_{0}$ is a field extension, then we write $X_{K}$ and $S_{K}$ instead of $X_{0} \otimes_{R_{0}} K$ and $S_{0} \otimes_{R_{0}} K$. If $K=\bar{K}$, then we simply write $X$ and $S$ instead of $X_{\bar{K}}$ or $S_{\bar{K}}$. The elements of $S$ are denoted by $x_{j}, j \in B_{0}$. From $₫ 2$ on we shall only consider the case $X_{0}=\mathbb{P}_{R_{0}}^{1}$ and $S_{0}=\{0,1, \infty\}$.

Let $G$ be a finite group and $f: Y \rightarrow X$ a $G$-cover of $X$ with branch locus $S$. We consider the set of ramification points of the cover $f$ as a marking on $Y$; this makes $Y$ a stably marked curve. Let $K$ be a finite extension of $K_{0}$ and $R$ the ring of integers of $K$. A model of $f$ over $K$ is a $G$-cover $f_{K}: Y_{K} \rightarrow X_{K}$ such that $f=f_{K} \otimes_{K} \bar{K}$. A good model of $f$ over $K$ is a model which extends to a finite and tame cover $f_{R}: Y_{R} \rightarrow X_{0} \otimes_{R_{0}} R$, ramified along $S_{0} \otimes_{R_{0}} R$ and étale everywhere else. We say that $f$ has good reduction if there exists a good model over some extension $K / K_{0}$. If $f$ does not have good reduction, we say that it has bad reduction.

The following is well known, see Bec89]. If $f$ has good reduction, then there exists a good model $f_{K_{0}}: Y_{K_{0}} \rightarrow X_{K_{0}}$ of $f$ over $K_{0}$, unique up to isomorphism. Moreover, if $p$ does not divide the order of the Galois group $G$, then $f: Y \rightarrow X$ has good reduction.

1.1.2. From now on, we assume that $f: Y \rightarrow X$ has bad reduction. Note that this implies that $p$ divides the order of $G$. If $K / K_{0}$ is a sufficiently large finite extension, then there exists a model $f_{K}: Y_{K} \rightarrow X_{K}$ of $f$ over $K$ such that $Y_{K}$ extends to a stably marked curve $Y_{R}$ over $R$. In particular, $Y_{R}$ is a semistable model of $Y_{K}$ over $R$ such that the ramification points of the cover $f_{K}$ specialize to pairwise disjoint, smooth points on the special fiber $\bar{Y}:=Y_{R} \otimes k$.

Since the stably marked model is unique, the action of the group $G$ on $Y_{K}$ extends to $Y_{R}$. Let $X_{R}:=Y_{R} / G$ be the quotient; then $X_{R}$ is a semistable curve 
over $R$ with generic fiber $X_{K}$, see Ray90, Appendix. Moreover, the branch points $x_{j}$ of $f_{K}$ specialize to pairwise distinct smooth points $\bar{x}_{j}$ on the special fiber $\bar{X}:=$ $X_{R} \otimes k$. We consider $\bar{X}$ as a marked semistable curve (note, however, that $\bar{X}$ is not stably marked). By Knu83, there exists a canonical contraction morphism $q_{R}: X_{R} \rightarrow X_{0} \otimes_{R_{0}} R$. Let $\bar{X}$ denote the special fiber of $X_{R}$. There is a unique irreducible component of $\bar{X}$ on which the induced map $\bar{q}: \bar{X} \rightarrow \bar{X}_{0}:=X_{0} \otimes_{R_{0}} k$ is an isomorphism. We may and will identify this component with $\bar{X}_{0}$. All other components of $\bar{X}$ are of genus 0 and are contracted by $\bar{q}$ to a closed point of $\bar{X}_{0}$.

Definition 1.1. The natural map $f_{R}: Y_{R} \rightarrow X_{R}$ is called the stable model of the Galois cover $f_{K}$. The induced map $\bar{f}: \bar{Y} \rightarrow \bar{X}$ on the special fibers is called the stable reduction of $f_{K}$. The component $\bar{X}_{0}$ of $\bar{X}$ is called the original component.

It is clear that the stable model is 'stable' under extension of the field $K$. In particular, the stable reduction does not depend on the choice of $K$. We remark that the stable model defined above differs slightly from the model used in Ray99. This difference is not essential, but certain results of Ray99 have to be reformulated. This is done in Lemma 1.4 below.

1.1.3. Following [DD97, we shall refer to $f: Y \rightarrow X$ as a $G$-cover (resp. as a mere cover) if we fix a group isomorphism $\operatorname{Aut}(Y / X) \cong G$ (resp. if we disregard such an isomorphism). In particular, an automorphism of the $G$-cover $f$ (resp. the mere cover $f$ ) is an automorphism of the curve $Y$ which commutes with the map $f$ and with the action of $G$ (resp. commutes with the map $f$ ). For an element $\sigma \in \operatorname{Gal}\left(\bar{K} / K_{0}\right)$ we denote by ${ }^{\sigma} f:{ }^{\sigma} Y \rightarrow X$ the conjugate $G$-cover. Let $\Gamma^{\text {in }}$ be the subgroup of $\operatorname{Gal}\left(\bar{K} / K_{0}\right)$ consisting of all elements $\sigma$ such that $f \cong{ }^{\sigma} f$, as $G$-covers. Similarly, let $\Gamma^{\mathrm{ab}}$ be the subgroup of $\operatorname{Gal}\left(\bar{K} / K_{0}\right)$ consisting of elements $\sigma$ such that ${ }^{\sigma} f \cong f$ as mere covers. Set

$$
K^{\mathrm{in}}:=\bar{K}^{\Gamma^{\mathrm{in}}}, \quad K^{\mathrm{ab}}:=\bar{K}^{\Gamma^{\mathrm{ab}}} .
$$

The field $K^{\text {in }}$ (resp. $K^{\text {ab }}$ ) is the field of moduli of the $G$-cover $f$ (resp. of the mere cover $f$ ), relative to the extension $\bar{K} / K_{0}$. See [DD97] for more details.

Let $K / K_{0}$ be a Galois extension such that $f$ has stable reduction over $K$, and let $f_{R}: Y_{R} \rightarrow X_{R}$ be the stable model. Also, let $\sigma \in \Gamma^{\mathrm{ab}}$. By definition of $\Gamma^{\mathrm{ab}}$, there exists a $\sigma$-linear automorphism $\kappa_{K, \sigma}: Y_{K} \stackrel{\sim}{\rightarrow} Y_{K}$ which normalizes the action of $G$ and induces the $\sigma$-linear automorphism $X_{K} \stackrel{\sim}{\rightarrow} X_{K}$ coming from the canonical $K_{0}$-structure on $X_{K}$. The automorphism $\kappa_{K, \sigma}$ is unique up to composition with an element of $G$. It extends to a $\sigma$-linear automorphism $\kappa_{R, \sigma}: Y_{R} \stackrel{\sim}{\rightarrow} Y_{R}$. Let $\bar{\kappa}_{\sigma}: \bar{Y} \stackrel{\sim}{\rightarrow}$ $\bar{Y}$ be the restriction of $\kappa_{R, \sigma}$ to the special fiber. This is a $k$-linear automorphism which normalizes the action of $G$. It is uniquely determined by $\sigma \in \Gamma^{\mathrm{ab}}$, up to composition with an element of $G$. We obtain a continuous homomorphism

$$
\bar{\kappa}^{\mathrm{ab}}: \Gamma^{\mathrm{ab}} \longrightarrow \operatorname{Aut}_{k}(\bar{Y}) / G,
$$

which we shall call the (absolute) monodromy action. Note that $\bar{\kappa}_{\sigma}$ induces an automorphism of $\bar{X}$ which is the identity on the original component. It follows from [DD97] that the set of $K^{\mathrm{ab}}$-models of the mere cover $f$ is in bijection with the set of homomorphisms $\Gamma^{\mathrm{ab}} \rightarrow \operatorname{Aut}_{k}(\bar{Y})$ which lift $\bar{\kappa}^{\mathrm{ab}}$. If a model $f_{K^{\mathrm{ab}}}$ of $f$ is specified, we will sometimes consider $\bar{\kappa}^{\mathrm{ab}}$ as a homomorphism $\Gamma^{\mathrm{ab}} \rightarrow \operatorname{Aut}_{k}(\bar{Y})$.

Let $\operatorname{Aut}_{G}(\bar{Y})$ denote the group of $k$-linear automorphisms of $\bar{Y}$ which commute with the $G$-action, and let $C_{G}$ denote the center of $G$. The restriction of $\bar{\kappa}^{\text {ab }}$ to $\Gamma^{\text {in }}$ 
yields a homomorphism

$$
\bar{\kappa}^{\text {in }}: \Gamma^{\text {in }} \longrightarrow \operatorname{Aut}_{G}(\bar{Y}) / C_{G},
$$

called the inner monodromy action. Similar to what we stated above, $K^{\mathrm{in}}$-models of the $G$-cover $f$ correspond one-to-one to lifts of $\bar{\kappa}^{\text {in }}$ to a homomorphism $\Gamma^{\text {in }} \rightarrow$ $\operatorname{Aut}_{G}(\bar{Y})$.

Let $\Gamma^{\text {st }}$ denote the subgroup of $\Gamma^{\mathrm{ab}}$ of elements $\sigma$ such that $\bar{\kappa}_{\sigma}^{\mathrm{ab}} \in G$, and set $K^{\text {st }}:=\bar{K}^{\Gamma^{\text {st }}}$. One shows that $K^{\text {st }} / K_{0}$ is the smallest extension over which $f$ has stable reduction. Compare with Ray99, §2.2.

\subsection{Mild reduction.}

1.2.1. Let $f: Y \rightarrow X$ be a $G$-cover as in 1.1. We assume that $f$ has bad reduction and denote by $\bar{f}: \bar{Y} \rightarrow \bar{X}$ its stable reduction. If $\eta$ is a point on $\bar{Y}$ (closed or generic), we denote by $G_{\eta}$ (resp. $I_{\eta}$ ) the corresponding decomposition (resp. inertia) group. By definition, $G_{\eta}$ is the set of elements of $G$ which stabilize $\eta$, and $I_{\eta}$ is the normal subgroup of $G_{\eta}$ consisting of elements which act trivially on the residue field $k(\eta)$.

Definition 1.2. We say that $f: Y \rightarrow \mathbb{P}^{1}$ has mild reduction if it has bad reduction and if the following two conditions hold for all points $\eta \in \bar{Y}$. First, the $p$-part of $I_{\eta}$ has at most order $p$. Second, if $I_{\eta} \neq 1$ then the order of $G_{\eta} / I_{\eta}$ is prime to $p$.

If $f: Y \rightarrow X$ has bad reduction and $p$ strictly divides the order of the Galois group $G$, then the reduction of $f$ is automatically mild. From now on, let us assume that $f: Y \rightarrow X$ has mild reduction.

1.2.2. Let us denote by $\left(\bar{X}_{v}\right)$ the family of irreducible components of the curve $\bar{X}$.

Definition 1.3. A component $\bar{X}_{v}$ of $\bar{X}$ which is not the original component and which is connected to the rest of $\bar{X}$ in a single point is called a tail. The point where a tail $\bar{X}_{v}$ meets the rest of $\bar{X}$ is denoted by $\infty_{v}$. If a tail $\bar{X}_{v}$ contains one of the points $\bar{x}_{j}, j \in B_{0}$, then $\bar{X}_{v}$ is called primitive. Otherwise, the tail $\bar{X}_{v}$ is called new. A component $\bar{X}_{v}$ which is not a tail is called an interior component.

For each component $\bar{X}_{v}$ of $\bar{X}$, we choose an irreducible component $\bar{Y}_{v}$ of $\bar{Y}$ above $\bar{X}_{v}$. We write $G_{v}$ for the decomposition group of $\bar{Y}_{v}$ and $I_{v}$ for the inertia group of $\bar{Y}_{v}$.

Lemma 1.4 (Raynaud). Suppose that $f: Y \rightarrow \mathbb{P}^{1}$ has mild reduction. Then the following hold.

(1) If $\bar{X}_{v}$ is a tail, then the inertia group $I_{v}$ is trivial. In other words, the map $\bar{Y}_{v} \rightarrow \bar{X}_{v}$ is a Galois cover, with Galois group $G_{v}$. If $\bar{X}_{v}$ is a primitive tail and contains the point $\bar{x}_{j}, j \in B_{0}$, then the cover $\bar{Y}_{v} \rightarrow \bar{X}_{v}$ is wildly ramified at $\infty_{v}$, tamely ramified at $\bar{x}_{j}$ and étale everywhere else. If $\bar{X}_{v}$ is a new tail, then $\bar{Y}_{v} \rightarrow \bar{X}_{v}$ is wildly ramified at $\infty_{v}$ and étale everywhere else.

(2) If $\bar{X}_{v}$ is not a tail, then the inertia group $I_{v}$ is cyclic of order $p$. The cover $\bar{Y}_{v} \rightarrow \bar{X}_{v}$ factors through the relative (k-linear) Frobenius $F: \bar{Y}_{v} \rightarrow \bar{Z}_{v}:=$ $\bar{Y}_{v}^{(p)}$. The resulting map $\bar{Z}_{v} \rightarrow \bar{X}_{v}$ is a Galois cover, with Galois group $H_{v}:=G_{v} / I_{v}$. It is étale outside the set of points which are either singular points of $\bar{X}$ or belong to the set $\left\{\bar{x}_{j} \mid j \in B_{0}\right\}$. 
(3) For $j \in B_{0}$, let $n_{j}$ be the ramification index of $f: Y \rightarrow X$ in $x_{j}$. Suppose that $n_{j}$ is prime to $p$. Then the specialization $\bar{x}_{j}$ of $x_{j}$ lies on a primitive tail $\bar{X}_{v}$, and the ramification index of $\bar{Y}_{v} \rightarrow \bar{X}_{v}$ at $\bar{x}_{j}$ is $n_{j}$.

(4) With the same notation as in (3), suppose that $n_{j}=p n_{j}^{\prime}$. Then $\bar{x}_{j}$ lies on a component $\bar{X}_{v}$ which is not a tail. Moreover, the Galois cover $\bar{Z}_{v} \rightarrow \bar{X}_{v}$ has ramification index $n_{j}^{\prime}$ at $\bar{x}_{j}$.

Proof. This is essentially proved in Ray99], $\S \S 2$ and 3 . There are two points where our assumptions differ from the assumptions made in Ray99. First, it is assumed in Ray99 that $p$ exactly divides the order of $G$. However, one only needs that $f$ has mild reduction. The second point is that (3) becomes false if we replace our definition of stable reduction with the definition used in Ray99. It is proved in BW00, Lemma 2.1.1 that (3) holds if we define $\bar{f}$ as in Definition 1.1.

\subsection{Deformation data.}

1.3.1. A priori, our definition of a deformation datum is independent of the study of stable reduction. So the following notation replaces, during $\$ 1.3 .1$, the notation introduced so far. Let $p$ be a prime number, $k$ an algebraically closed field of characteristic $p$ and $X$ a smooth, projective and irreducible curve over $k$.

Definition 1.5. A deformation datum over $X$ is a pair $(Z, \omega)$, where $Z \rightarrow X$ is a tame (connected) Galois cover and $\omega$ is a meromorphic differential form on $Z$ such that the following hold.

(1) The order of the Galois group $H=\operatorname{Gal}(Z / X)$ is prime to $p$.

(2) We have

$$
\beta^{*} \omega=\chi(\beta) \cdot \omega, \quad \text { for all } \beta \in H,
$$

where $\chi: H \rightarrow \mathbb{F}_{p}^{\times}$is a character with values in the prime field $\mathbb{F}_{p}$.

(3) The differential $\omega$ is either logarithmic (i.e. of the form $\omega=\mathrm{d} u / u$ ) or exact (i.e. $\omega=\mathrm{d} u$ ). In the first case, the deformation datum $(Z, \omega)$ is called multiplicative, in the second case it is called additive.

The pair $(H, \chi)$ is called the type of the deformation datum $(Z, \omega)$.

Let $\xi \in Z$ be a closed point and $\tau \in X$ its image. Denote the stabilizer of $\xi$ by $H_{\xi} \subset H$. We attach to the triple $(Z, \omega, \tau)$ the following three invariants:

$$
m_{\tau}:=\left|H_{\xi}\right|, \quad h_{\tau}:=\operatorname{ord}_{\xi}(\omega)+1, \quad \sigma_{\tau}:=h_{\tau} / m_{\tau} .
$$

It is clear that $m_{\tau}, h_{\tau}$ and $\sigma_{\tau}$ depend only on $\tau$ but not on $\xi$, and that $\left(m_{\tau}, h_{\tau}\right)=$ $(1,1)$ for all but a finite number of points $\tau \in X$. If $\left(m_{\tau}, h_{\tau}\right) \neq(1,1)$, then $\tau$ is called a critical point for the deformation datum $(Z, \omega)$.

Given a deformation datum $(Z, \omega)$, we often use the following or a similar notation. Let $\left(\tau_{j}\right)_{j \in J}$ be the family of critical points for $(Z, \omega)$, indexed by the finite set $J$. For $j \in J$, we write $m_{j}, h_{j}$ and $\sigma_{j}$ instead of $m_{\tau_{j}}, h_{\tau_{j}}$ and $\sigma_{\tau_{j}}$. The data $\left(m_{j} ; h_{j}\right)_{j \in B}$ is called the signature of the deformation datum $(Z, \omega)$. A straightforward computation shows that

$$
\sum_{j \in J}\left(\sigma_{j}-1\right)=2 g_{X}-2
$$

(where $g_{X}$ denotes the genus of the curve $X$ ). 
Remark 1.6. Let $\tilde{Z}:=Z / \operatorname{Ker}(\chi)$. It is easy to see that $\omega$ descends to a differential $\tilde{\omega}$ on $\tilde{Z}$ and that $(\tilde{Z}, \tilde{\omega})$ is again a deformation datum, with critical points $\tau_{j}$ and invariants

$$
\tilde{m}_{j}=\frac{m_{j}}{\left|\operatorname{Ker}(\chi) \cap H_{j}\right|}, \quad \tilde{h}_{j}=\frac{h_{j}}{\left|\operatorname{Ker}(\chi) \cap H_{j}\right|}, \quad \tilde{\sigma}_{j}=\frac{\tilde{h}_{j}}{\tilde{m}_{j}}=\sigma_{j} .
$$

Here $H_{j} \subset H$ denotes the stabilizer of a point $\xi_{j}$ above $\tau_{j}$.

1.3.2. Let us now return to our original notation, concerning the Galois cover $f: Y \rightarrow X$ and its stable reduction $\bar{f}: \bar{Y} \rightarrow \bar{X}$. Let $\bar{X}_{v}$ be an interior component of $\bar{X}$ (see Definition [1.3). Following [Hen00], we define a deformation datum $\left(\bar{Z}_{v}, \omega_{v}\right)$ over $\bar{X}_{v}$, as follows.

Choose an irreducible component $\bar{Y}_{v}$ of $\bar{Y}$ above $\bar{X}_{v}$. By Lemma 1.4 (2), the inertia group $I_{v}$ of $\bar{Y}_{v}$ is cyclic of order $p$ and the quotient group $H_{v}:=G_{v} / I_{v}$ is of prime-to- $p$ order. Moreover, the cover $\bar{Y}_{v} \rightarrow \bar{X}_{v}$ is the composition of a purely inseparable cover $\bar{Y}_{v} \rightarrow \bar{Z}_{v}$ of degree $p$ and an $H_{v}$-Galois cover $\bar{Z}_{v} \rightarrow \bar{X}_{v}$. Let $\hat{Y}_{v}:=\operatorname{Spec} \hat{\mathcal{O}}_{Y_{R}, v}$ be the completion of $Y_{R}$ at the generic point of the component $\bar{Y}_{v}$; thus $\hat{\mathcal{O}}_{Y_{R}, v}$ is a complete discrete valuation ring with residue field $k\left(\bar{Y}_{v}\right)$. The action of $I_{v}$ on $Y_{R}$ induces an action on $\hat{Y}_{v}$. Let $\hat{Z}_{v}:=\hat{Y}_{v} / I_{v}$ be the quotient; we have $\hat{Z}_{v}=$ Spec $A$ for a complete discrete valuation ring $A$ with residue field $k\left(\bar{Z}_{v}\right)$. We may assume that the field $K$ contains the $p$ th roots of unity. Let us choose an isomorphism $I_{v} \cong \boldsymbol{\mu}_{p}(K)$ between $I_{v}$ and the group of $p$ th roots of unity. By Ray99, $§ 1.2 .1$, the map $\hat{Y}_{v} \rightarrow \hat{Z}_{v}$ is a torsor under a finite flat $R$-group scheme $\mathcal{G}$ of rank $p$, such that $\mathcal{G} \otimes_{R} K=\boldsymbol{\mu}_{p}$ acts on $\hat{Y}_{v} \otimes_{R} K$ via the isomorphism $I_{v} \cong \boldsymbol{\mu}_{p}(K)$. By Kummer theory, the $\boldsymbol{\mu}_{p}$-torsor $\hat{Y}_{v} \otimes_{R} K \rightarrow \hat{Z}_{v} \otimes_{R} K$ is given by a Kummer equation $y^{p}=u$, with $u \in A$.

To define the differential $\omega_{v}$, we have to distinguish two cases. First, let us assume that $\mathcal{G} \otimes_{R} k \cong \boldsymbol{\mu}_{p}$. This happens if and only if we may choose the Kummer equation $y^{p}=u$ such that the residue $\bar{u}$ of $u$ is not a $p$ th power. In this case, we set $\omega_{v}:=\mathrm{d} \bar{u} / \bar{u}$ and we say that the cover $f$ has multiplicative reduction at $\bar{Y}_{v}$. Otherwise $\mathcal{G} \otimes_{R} k \cong \boldsymbol{\alpha}_{p}$ and we say that $f$ has additive reduction at $\bar{Y}_{v}$. In this case we can choose the equation $y^{p}=u$ such that $u=1+\pi^{p} w$, with $\pi \in R, w \in A$ and $0<v_{R}(\pi)<v_{R}(p) /(p-1)$, and such that the residue $\bar{w}$ of $w$ is not a $p$ th power. We set $\omega_{v}:=\mathrm{d} \bar{w}$. It is clear that $\omega_{v}$ depends on the choice of the isomorphism $I_{v} \cong \boldsymbol{\mu}_{p}(K)$. Indeed, composing this isomorphism with the automorphism $\zeta_{p} \mapsto \zeta_{p}^{a}$ (where $a \in \mathbb{F}_{p}^{\times}$) amounts to multiplying $\omega_{v}$ by $a$. Let $\chi_{v}: H_{v} \rightarrow \mathbb{F}_{p}^{\times}$be the character describing the action of $H_{v}$ on $I_{v}$ by conjugation. It is clear that $\beta^{*} \omega_{v}=\chi_{v}(\beta) \cdot \omega_{v}$ for all $\beta \in H_{v}$. Therefore, $\left(\bar{Z}_{v}, \omega_{v}\right)$ is a deformation datum.

By the work of Henrio [Hen00], the critical points of the deformation datum $\left(\bar{Z}_{v}, \omega_{v}\right)$ are precisely the points of $\bar{X}_{v}$ which are either singular on $\bar{X}$ or specialization of a branch point of $f_{K}$ with ramification index divisible by $p$. More precisely, we have

Proposition 1.7. Over each interior component $\bar{X}_{v}$ we obtain a deformation $d a-$ tum $\left(\bar{Z}_{v}, \omega_{v}\right)$, with the following properties. If $\tau \in \bar{X}_{v}$ is a critical point for $\left(\bar{Z}_{v}, \omega_{v}\right)$ then one of the following two statements hold.

(1) The point $\tau \in \bar{X}_{v}$ is a singular point of $\bar{X}$.

(2) The point $\tau$ is the specialization of a point $x_{j} \in S_{0}$ at which the cover $f$ has ramification of order divisible by $p$. 
In Case (1), we have $\sigma_{\tau} \notin\{0,1\}$. In Case (2), we have $\sigma_{\tau}=0$ and $\left(\bar{Z}_{v}, \omega_{v}\right)$ is multiplicative.

1.3.3. By Proposition 1.7, the stable reduction $\bar{f}: \bar{Y} \rightarrow \bar{X}$ of a $G$-cover $f$ : $Y \rightarrow X$ with mild reduction is naturally equipped with a collection of deformation data $\left(\bar{Z}_{v}, \omega_{v}\right)$. These deformation data are related among each other by certain compatibility conditions. To formulate these conditions, let $\tau$ be a singular point of $\bar{X}$, and let $\bar{X}_{v_{1}}, \bar{X}_{v_{2}}$ be the two components of $\bar{X}$ which intersect in $\tau$. For $i=1,2$, we define a pair $\left(m_{i}, h_{i}\right)$ as follows.

- If $\bar{X}_{v_{i}}$ is an interior component, we define $\left(m_{i}, h_{i}\right)$ as the pair of invariants attached to $\left(\bar{Z}_{v_{i}}, \omega_{v_{i}}, \tau\right)$.

- Suppose that $\bar{X}_{v_{i}}=\bar{X}_{j}$ is a tail of $\bar{X}$. Let $\eta \in \bar{Y}_{j}$ be a point lying above $\tau$ and let $I_{\eta} \subset G$ be the inertia group at $\eta$. We define $m_{i}$ as the order of the maximal prime-to- $p$ quotient of $I_{\eta}$. Furthermore, we let $h_{i}$ be the conductor of $I_{\eta}$, i.e. the unique integer such that $\left|I_{\eta, h_{i}}\right|=p$ and $I_{\eta, h_{i}+1}=\{1\}$. (Here $\left(I_{\eta, \nu}\right)_{\nu \geq 0}$ denotes the filtration of higher ramification groups.)

Proposition 1.8. We have $m_{1}=m_{2}$ and $h_{1}=-h_{2}$.

Proof. The equality $m_{1}=m_{2}$ is trivial. Let $\hat{Y}:=\operatorname{Spec} \hat{\mathcal{O}}_{Y_{R}, \eta}$ be the completion of $Y_{R}$ at $\eta$. The $R$-scheme $\hat{Y}$ is a formal annulus and is acted upon by $I_{\eta}$. The invariants $h_{1}$ and $h_{2}$ can be defined purely in terms of this local action, and the equality $h_{1}=-h_{2}$ is proved in [Hen00], Proposition 1.10. (In [Hen00], the invariant $h$ is called $-m$.)

Remark 1.9. It seems plausible that the existence of a set of deformation data satisfying these compatibility conditions gives a necessary and sufficient condition for a map $\bar{f}: \bar{Y} \rightarrow \bar{X}$ between semistable curves to occur as the stable reduction of a Galois cover between smooth curves in characteristic 0 . That this is true for three point covers is proved in 4 . In the case of $p$-cyclic covers, results in this direction are obtained in [Hen00] and Saï01.

1.4. The vanishing cycle formula. The vanishing cycle formula, proved by Raynaud in Ray99, is the key formula which controls the (local) invariants describing the bad reduction occurring over the tails by a global expression. In this subsection we give another proof. The main observation is that (2) gives a 'local' vanishing cycle formula for each interior component of $\bar{X}$. The global vanishing cycle formula is then easily obtained from its local version using the compatibility relation of Proposition 1.8 and induction over the tree of components of $\bar{X}$. We use this occasion to set up some more notation which may seem at bit technical at first but will pay off in $\$ 2.1$.

1.4.1. Recall that an unordered graph is given by a quintuple $\left(V, E, s, t,^{-}\right)$. Here $V$ is the set of vertices, $E$ is the set of (ordered) edges, $s, t: E \rightarrow V$ are the source and the target map, and $E \stackrel{\sim}{\rightarrow} E, e \mapsto \bar{e}$, is a fixed point free involution of $E$ such that $s(\bar{e})=t(e)$ for all $e \in E$.

Let $T^{\prime}=\left(V^{\prime}, E^{\prime}, s, t,^{-}\right)$be the dual graph of the semistable curve $\bar{X}$. Thus, a vertex $v \in V^{\prime}$ corresponds to an irreducible component $\bar{X}_{v}$ of $\bar{X}$, and an edge $e \in E^{\prime}$ corresponds to a triple $\left(\bar{x}, v_{1}, v_{2}\right)$ such that the (distinct) components $\bar{X}_{v_{1}}$ and $\bar{X}_{v_{2}}$ intersect in the (singular) point $\bar{x}$. Let $B_{\text {wild }} \subset B_{0}$ be the set of indices $j$ such that ramification index of the Galois cover $f: Y \rightarrow X$ at $x_{j}$ is divisible by 
$p$. We define $T=\left(V, E, s, t,{ }^{-}\right)$as the unordered graph obtained from $T^{\prime}$ by adding a vertex for each point $x_{j}$ with $j \in B_{\text {wild }}$. More precisely, $V:=V^{\prime} \cup B_{\text {wild }}$ and a vertex $j \in B_{\text {wild }}$ is connected to the vertex $v \in V^{\prime}$ by an edge $e \in E$ if and only if the point $x_{j} \in S_{0}$ specializes to the component $\bar{X}_{v}$. Note that $T$ is a connected tree. Also, to each edge $e$ of $T$ corresponds a closed point $\bar{x}_{e} \in \bar{X}$, as follows. If the edge $e$ joins two vertices $v_{1}, v_{2} \in V^{\prime}$, then $\bar{x}_{e}$ is the point where $\bar{X}_{v_{1}}$ intersects $\bar{X}_{v_{2}}$. If $e$ joins two vertices $j \in B_{\text {wild }}$ and $v \in V^{\prime}$, then $\bar{x}_{e}:=\bar{x}_{j} \in \bar{X}_{v}$ is the point to which $x_{j}$ specializes.

We consider the vertex $v_{0}$ corresponding to the original component $\bar{X}_{v_{0}}$ as the root of the tree $T$. Also, we denote by $B$ the set of leaves of $T$, i.e. the set of vertices $v \in V-\left\{v_{0}\right\}$ which are adjacent to a unique vertex $v^{\prime}$. Thus, a vertex $v \in V_{\text {int }}:=$ $V-B$ corresponds to an interior component $\bar{X}_{v}$. By definition, $B_{\text {wild }} \subset B$. An element $j$ in $B_{\text {prim }}=B_{0}-B_{\text {wild }}$ corresponds to a ramification point $x_{j}$ of $f$ of order prime-to- $p$. By Lemma 1.4, $x_{j}$ specializes to a point $\bar{x}_{j}$ on a primitive tail $\bar{X}_{v}$. The resulting map $B_{\text {prim }} \rightarrow B$ is clearly injective. Therefore, we may and will consider $B_{0}=B_{\text {prim }} \cup B_{\text {wild }}$ as a subset of $B$. Then $B_{\text {new }}:=B-B_{0}$ is the set of leaves corresponding to the new tails of $\bar{X}$.

1.4.2. We shall now attach integers $m_{e}, h_{e}$ and a rational number $\sigma_{e}$ to each edge $e$ of the tree $T$. Suppose first that $v:=s(e)$ is an interior vertex. Let $\left(\bar{Z}_{v}, \omega_{v}\right)$ be the deformation datum over $\bar{X}_{v}$, as defined in $\$ 1.3 .2$. Note that $\bar{x}_{e} \in \bar{X}_{v}$ is a critical point for $\left(\bar{Z}_{v}, \omega_{v}\right)$, by Proposition [1.7 We define the numbers $m_{e}, h_{e}$ and $\sigma_{e}$ as the invariants attached to the triple $\left(\bar{Z}_{v}, \omega_{v}, \bar{x}_{e}\right)$. If $v:=s(e)$ is a leaf of $T$, then we set $m_{e}:=m_{\bar{e}}, h_{e}:=-h_{\bar{e}}$ and $\sigma_{e}:=-\sigma_{\bar{e}}$. Equation (2) and Proposition 1.8 imply the following.

Proposition 1.10. For all interior vertices $v$ of $T$ we have

$$
\sum_{s(e)=v}\left(\sigma_{e}-1\right)=2 g_{v}-2
$$

(where $g_{v}$ is the genus of $\bar{X}_{v}$ ). Furthermore, for all edges e of $T$ we have

$$
\sigma_{e}+\sigma_{\bar{e}}=0 \text {. }
$$

For each leaf $j \in B$, let $e_{j}$ be the unique edge with target $j$. We set $m_{j}:=m_{e_{j}}$, $h_{j}:=m_{e_{j}}$ and $\sigma_{j}:=\sigma_{e_{j}}$. If $j \notin B_{\text {wild }}$, then $m_{j}$ is the prime-to- $p$ part of the ramification index of the Galois cover $\bar{Y}_{j} \rightarrow \bar{X}_{j}$ at $\bar{x}_{e}=\infty_{j}$ and $h_{j}$ is the conductor of the wild ramification in that point, see Proposition 1.8. Therefore, the rational number $\sigma_{j}=h_{j} / m_{j}$ is the ramification invariant introduced in [Ray99], §1.1. It is equal to the jump in the filtration of higher ramification groups for the branch point $\infty_{j} \in \bar{X}_{j}$, with respect to the upper numbering, see [Ser68]. Note that we have defined $\sigma_{j}=0$ for $j \in B_{\text {wild }}$. We shall call the tuple $\left(\sigma_{j}\right)_{j \in B}$ the signature of the stable reduction $\bar{f}: \bar{Y} \rightarrow \bar{X}$. It is shown in Ray99 that $\sigma_{j}>1$ for $j \in B_{\text {new }}$. Moreover, we have the following.

Corollary 1.11 (The vanishing cycle formula). The signature $\left(\sigma_{j}\right)_{j \in B}$ of $\bar{f}$ satisfies

$$
\sum_{j \in B_{\mathrm{prim}}} \sigma_{j}+\sum_{j \in B_{\text {new }}}\left(\sigma_{j}-1\right)=2 g_{X}-2+\left|B_{0}\right| .
$$

Proof. This is proved in Ray99, §3.4. Here is another proof, using Proposition 1.10. Let $T_{0} \subset T_{1} \subset \ldots \subset T_{n}$ be a chain of subtrees of $T$ such that $T_{0}=\left\{v_{0}\right\}$, 
$T_{i}=T_{i-1} \cup\left\{v_{i}\right\}$ and $T_{n}=T-B$. Let $E_{i}$ denote the set of edges of $T$ such that $s(e) \in T_{i}$ and $t(e) \notin T_{i}$. We claim that $\sum_{e \in E_{i}}\left(\sigma_{e}-1\right)=2 g_{X}-2$ holds for all $0 \leq i \leq n$. Indeed, Proposition 1.10 shows that the claim is true for $i=0$ and then, by induction, for all $i$. For $i=n$, the claim is equivalent to (5).

\section{The Case of three Point COVERS}

In this section we apply the results of the last section to the case of three point covers. The main result is Theorem [2.6, which corresponds to Theorem 2 of the Introduction. This theorem essentially states that the stable reduction of a three point cover with mild reduction is as simple as possible. The idea underlying its proof is that the stable reduction $\bar{f}: \bar{Y} \rightarrow \bar{X}$, together with the associated deformation data, should be 'rigid' objects, i.e. should not allow any nontrivial deformation in equal characteristic. This imposes strong restrictions on the deformation data $\left(\bar{Z}_{v}, \omega_{v}\right)$ attached to $\bar{f}$. We verify these rigidity restrictions somewhat indirectly, using the local and global vanishing cycle formulas. Then a key lemma from [Wew03 shows that $\bar{f}$ can support no additive deformation datum, and only a single multiplicative one, proving Theorem 2.6 To make this proof work one has to exclude two exceptional cases. However, this is no real restriction, because all our final results are easily seen to hold for these exceptional cases as well.

In $₫ 2.2$ we define the notion of a special $G$-deformation datum. To such an object we can associate a map $\bar{f}: \bar{Y} \rightarrow \bar{X}$ which looks like the stable reduction of a three point $G$-cover, and which we call a special $G$-map. Later, in $\$ 4$, we will show that every special $G$-map actually arises as the stable reduction of some three point cover.

\subsection{The structure of $\bar{f}$.}

2.1.1. We continue with the notation introduced in \$1 In addition, we assume that $X_{0}=\mathbb{P}_{R_{0}}^{1}$ and $S_{0}=\{0,1, \infty\}$. Note that the right hand side of the vanishing cycle formula (5) is equal to 1 .

Definition 2.1. We say that the stable reduction of $f$ is exceptional if one of the following two cases occurs.

(1) We have $B_{\text {prim }}=\left\{j_{0}\right\}, B_{\text {new }}=\emptyset$ and $\sigma_{j_{0}}=1$.

(2) We have $B_{\text {prim }}=\emptyset, B_{\text {new }}=\left\{j_{0}\right\}$ and $\sigma_{j_{0}}=2$.

Proposition 2.2. Let $f: Y \rightarrow X=\mathbb{P}_{\bar{K}}^{1}$ be a Galois cover ramified at $\{0,1, \infty\}$, with exceptional reduction. Then the curve $\bar{X}$ consists only of the original component $\bar{X}_{0}$ and the unique tail $\bar{X}_{j_{0}}$.

Proof. We will prove the proposition only for case (2) of Definition 2.1 and leave case (1) to the reader. Let $T^{\prime}$ be the dual graph of the curve $\bar{X}$, considered as a subtree of $T$. By assumption, $T^{\prime}$ has a unique leaf $j_{0}$. Therefore, $T^{\prime}$ is simply a chain going from the root $v_{0}$ to $j_{0}$. Let $v$ be the unique vertex of $T^{\prime}$ adjacent to the leaf $j_{0}$ and let $e$ be the edge with source $v$ and target $j_{0}$. This means that the interior component $\bar{X}_{v}$ intersects the unique (new) tail $\bar{X}_{j_{0}}$ in the point $\bar{x}_{e}$. We have to show that $v=v_{0}$, i.e. that $\bar{X}_{v}$ is the original component $\bar{X}_{0}$. So, let us assume that $v \neq v_{0}$. Then there exists a unique edge $e^{\prime} \neq e$ with source $v$, corresponding to a point $\bar{x}_{e^{\prime}}$ where $\bar{X}_{v}$ intersects another component $\bar{X}_{v^{\prime}}$. Let us first suppose that there exists another edge $e^{\prime \prime}$ (of the tree $T$ ) with source $v$ besides $e$ and $e^{\prime}$. Then $t\left(e^{\prime \prime}\right) \in B_{\text {wild }}$, i.e. the point $\bar{x}_{e^{\prime \prime}}$ is the specialization of a branch point of $f$ with 
ramification index divisible by $p$. Since $\bar{X}_{v}$ is not the original component, there can be at most one branch point specializing to $\bar{X}_{v}$. Therefore, $e, e^{\prime}, e^{\prime \prime}$ are the only edges with source $v$. Since $\sigma_{e}=2$ and $\sigma_{e^{\prime \prime}}=0$, (3) shows that $\sigma_{e^{\prime}}=-1$. This means that the differential $\omega_{v}$ has a pole of order $\geq 2$ at each point of $\bar{Z}_{v}$ lying above $\bar{x}_{e^{\prime}}$. This shows that $\omega_{v}$ cannot be logarithmic. On the other hand, $\omega_{v}$ has a simple pole at each point above $\bar{x}_{e^{\prime \prime}}$, so $\omega_{v}$ cannot be exact. This is a contradiction. We may therefore assume that $e$ and $e^{\prime}$ are the only edges with source $v$. It follows that the cover $\bar{Z}_{v} \rightarrow \bar{X}_{v}$ is ramified at most at two points; in particular, the genus of $\bar{Z}_{v}$, and therefore the genus of $\bar{Y}_{v}$ as well, is zero. Moreover, $\bar{Y}_{v}$ intersects the rest of $\bar{Y}$ in two points and no ramification point specializes to $\bar{Y}_{v}$. Thus, the 'three point condition' does not hold for $\bar{Y}$, in contradiction with the definition of the stable model in 1.1 . The proposition follows.

Remark 2.3. It may happen that the Galois cover $f: Y \rightarrow X$ has bad reduction but that the curve $Y$ has good reduction. If this is the case, then the stable reduction of $f$ is exceptional.

2.1.2. For the rest of 22.1 we assume that the reduction of $f$ is not exceptional. The vanishing cycle formula (5), together with the inequalities $\sigma_{j}>0$ for $j \in B_{\text {prim }}$ and $\sigma_{j}>1$ for $j \in B_{\text {new }}$, immediately implies the inequalities

$$
0<\sigma_{j}<1 \quad \text { for } j \in B_{\text {prim }}, \quad 1<\sigma_{j}<2 \text { for } j \in B_{\text {new }} \text {. }
$$

Moreover, $\left|B_{\text {prim }} \cup B_{\text {new }}\right| \geq 2$. For an edge $e$ of $T$ we define $\nu_{e}:=\left\lfloor\sigma_{e}\right\rfloor$ to be the largest integer less than or equal to the rational number $\sigma_{e}$. Note that we have $\sigma_{e}=\nu_{e}+\left\langle\sigma_{e}\right\rangle$, where $\left\langle\sigma_{e}\right\rangle \geq 0$ denotes the fractional part of $\sigma_{e}$. For a leaf $j \in B$, we write $\nu_{j}:=\left\lfloor\sigma_{j}\right\rfloor$. By (6) we have $\nu_{j}=0$ for $j \in B_{0}$ and $\nu_{j}=1$ for $j \in B_{\text {new }}$. Since $\left|B_{0}\right|=3$, the vanishing cycle formula (5) implies

$$
\sum_{j \in B}\left\langle\sigma_{j}\right\rangle=1
$$

Recall that $T^{\prime}$ denotes the dual graph of the curve $\bar{X}$, considered as subtree of $T$. Let $E^{\prime}$ denote the set of edges of $T^{\prime}$. Moreover, let $E_{\mathrm{int}}^{\prime} \subset E^{\prime}$ be the set of edges connecting two interior vertices.

\section{Proposition 2.4.}

(1) For all edges $e \in E^{\prime}$ we have $\nu_{e}+\nu_{\bar{e}}=-1$.

(2) For all interior vertices $v \in V_{\text {int }}$ we have

$$
\sum_{s(e)=v}\left\langle\sigma_{e}\right\rangle=1 \quad \text { and } \quad \sum_{s(e)=v}\left(\nu_{e}-1\right)=-3 .
$$

Proof. For $e \in E^{\prime}$ define

$$
d_{e}:=\left\langle\sigma_{e}\right\rangle+\left\langle\sigma_{\bar{e}}\right\rangle-1=-\nu_{e}-\nu_{\bar{e}}-1 .
$$

The last equality follows from (4). Clearly, $d_{e}=d_{\bar{e}}, d_{e} \in\{-1,0\}$, and $d_{e}=-1$ if and only if $\sigma_{e} \in \mathbb{Z}$. For $v \in V_{\text {int }}$, define

$$
c_{v}:=-1+\sum_{s(e)=v}\left\langle\sigma_{e}\right\rangle=-3+\sum_{s(e)=v}\left(\nu_{e}-1\right) .
$$

The last equality is a consequence of (3). It is easy to see that $c_{v} \in\{-1,0,1, \ldots\}$ and that $c_{v}=-1$ if and only if $d_{e}=-1$ for all $e \in E^{\prime}$ such that $s(e)=v$. We have to show that $d_{e}=c_{v}=0$. 
It is convenient to introduce the following terminology. A vertex $v$ of $T$ is said to precede an edge $e$ (notation: $v \prec e$ ) if $v$ lies in the connected component of $T-\{e\}$ which contains $s(e)$. An edge $e^{\prime}$ is said to precede another edge $e$ (notation: $e^{\prime} \prec e$ ) if $t\left(e^{\prime}\right) \prec e$. Note that ' $\prec$ ' is a partial order on the set $E$ of edges.

Suppose that there exists an edge $e \in E^{\prime}$ with $d_{e} \neq 0$. We may suppose that $e$ is minimal with this property, i.e. $d_{e^{\prime}}=0$ for all edges $e^{\prime} \in E^{\prime}$ which precede $e$. We claim that $c_{v} \geq 0$ for all $v \in V_{\text {int }}$ which preced $e$. Indeed, since $\bar{X}$ has at least two tails, there exist at least two (distinct) edges in $E^{\prime}$ with target $v$. At least one of them precedes $e$. Hence there exists an edge $e^{\prime} \in E^{\prime}$ with $d_{e^{\prime}}=0$ and $t(e)=v$. This proves the claim. As a consequence, we obtain the inequality

$$
\begin{gathered}
0 \leq \sum_{\substack{v \in V_{\mathrm{int}} \\
v \prec e}} c_{v}=\left\langle\sigma_{e}\right\rangle+\left(\sum_{\substack{e^{\prime} \in E_{\mathrm{int}}^{\prime} \\
e^{\prime} \prec e}} d_{e^{\prime}}\right)+\left(\sum_{\substack{j \in B \\
j \prec e}}\left\langle\sigma_{j}\right\rangle\right)-1 \\
=\left\langle\sigma_{e}\right\rangle+\left(\sum_{\substack{j \in B \\
j \prec e}}\left\langle\sigma_{j}\right\rangle\right)-1 .
\end{gathered}
$$

Since the curve $\bar{X}$ has at least two tails, there exists $j \in B_{\text {prim }} \cup B_{\text {new }}$ such that $j$ does not precede $e$. Moreover, $\sigma_{j}>0$. Therefore, (8) implies $\left\langle\sigma_{e}\right\rangle>0$. But this is a contradiction to the assumption $d_{e} \neq 0$. We conclude that $d_{e}=0$ for all $e \in E^{\prime}$. It remains to prove $c_{v}=0$ for all $v \in V_{\text {int }}$. The same argument we used above together with $d_{e}=0$ for all $e \in E^{\prime}$ shows that $c_{v} \geq 0$ for all $v \in V_{\text {int }}$. Now the proposition follows from

$$
\sum_{v \in V_{\mathrm{int}}} c_{v}=\left(\sum_{e \in E_{\mathrm{int}}^{\prime}} d_{e}\right)+\left(\sum_{j \in B}\left\langle\sigma_{j}\right\rangle\right)-1=0 .
$$

Lemma 2.5. Suppose that $\bar{f}$ is not exceptional, and let e be an edge of $T$. Then

$$
-2 \leq \nu_{e} \leq 1
$$

Moreover, $\nu_{e} \geq 0$ if and only if the root $v_{0}$ precedes $e$. In particular, if $v$ is an interior vertex not equal to $v_{0}$, then there exists a unique edge $e$ with source $v$ such that $\nu_{e}<0$.

Proof. (Compare to [Wew03], Lemma 2.6.) Using Proposition 2.4 and induction in the tree $T$, one shows that

$$
\nu_{e}=1-\left|\left\{j \in B_{0} \mid j \succ e\right\}\right| .
$$

Note that $\left|B_{0}\right|=3$ and that the root $v_{0}$ is the median of the set of leaves $B_{0}$. The lemma follows immediately.

2.1.3. We can now prove our first main result. It applies in particular if $p$ strictly divides the order of $G$.

Theorem 2.6. Let $f: Y \rightarrow X=\mathbb{P}_{\bar{K}}^{1}$ be a Galois cover ramified at $\{0,1, \infty\}$, with bad reduction. If $f$ has mild reduction (Definition 1.2) then the curve $\bar{X}$ consists only of the original component $\bar{X}_{0}$ and the tails $\bar{X}_{j}, j \in B_{\text {prim }} \cup B_{\text {new }}$. 
Proof. If $B_{\text {wild }}=\emptyset$ then this follows from Wew03, Theorem 2.1, using Raynaud's construction of the auxiliary cover. The proof we give below follows the same lines, but avoids the construction of the auxiliary cover.

We prove the theorem by contradiction. Assume that there exists an interior vertex $v$ of $T$ other than the root $v_{0}$. By Proposition 2.2, the reduction of $f$ is not exceptional. We may assume that $v$ is adjacent to the root $v_{0}$, i.e. there exists an edge $e_{0}$ with source $v$ and target $v_{0}$. This means that the component $\bar{X}_{v}$ intersects the original component $\bar{X}_{0}$ in the point $\bar{x}_{e_{0}}$. Let $\left(\bar{Z}_{v}, \omega_{v}\right)$ be the deformation datum over $\bar{X}_{v}$ as defined in 1.3 .2 For the rest of this proof, $e$ runs over the set of edges with source $v$. By Proposition 2.4 we have $\sum_{e}\left\langle\sigma_{e}\right\rangle=1$ and $\sum_{e}\left(\nu_{e}-1\right)=-3$. Also, Lemma 2.5 implies that $-2 \leq \nu_{e} \leq 1$ for all $e$, and $\nu_{e}<0$ if and only if $e=e_{0}$. In this situation, Wew03, Lemma 2.8 says that $\omega_{v}$ cannot be exact (actually, in Wew03. one assumes that the type of $\left(\bar{Z}_{v}, \omega_{v}\right)$ has an injective character $\chi_{v}$; the general case follows from Remark 1.6). Therefore, $\omega_{v}$ is logarithmic. On the other hand, since $\nu_{e_{0}}<0$, the differential has a pole of order $\geq 2$ at each point of $\bar{Z}_{v}$ lying above $\bar{x}_{e_{0}}$. Therefore, $\omega_{v}$ cannot be logarithmic. This gives the desired contradiction and proves the theorem.

2.2. Special $G$-deformation data. In this section we define special $G$-deformation data. To a special $G$-deformation datum we then associate a special $G$-map $\bar{f}: \bar{Y} \rightarrow \bar{X}$. Briefly, a special $G$-map is a map between semistable curves over $k$ which looks like the stable reduction of a three point $G$-cover with mild reduction.

2.2.1. Let $k$ be an algebraically closed field of characteristic $p>0$ and $\bar{X}$ a smooth projective curve over $k$ of genus 0 . Let $(\bar{Z}, \omega)$ be a deformation datum over $\bar{X}$, of type $(H, \chi)$. As usual, we denote by $\left(\tau_{j}\right)_{j \in B}$ the set of critical points for $(\bar{Z}, \omega)$. Also, for $j \in B$ we denote by $m_{j}, h_{j}$ and $\sigma_{j}$ the invariants attached to $\left(\bar{Z}, \omega, \tau_{j}\right)$. See $\$ 1.3$

Definition 2.7. The deformation datum $(\bar{Z}, \omega)$ is called special if for all $j \in B$ we have $\sigma_{j}<2$ and $\sigma_{j} \neq 1$ and if there exists a subset $B_{0} \subset B$ with exactly three elements such that $\sigma_{j}<1$ if and only if $j \in B_{0}$.

Remark 2.8. In case the character $\chi$ is injective and $\sigma_{j}>0$ we recover the definition of a special deformation datum given in [Wew03].

In the following we assume that $(\bar{Z}, \omega)$ is special. Let $B_{\text {wild }}$ (resp. $B_{\text {prim }}$, resp. $B_{\text {new }}$ ) denote the set of all indices $j \in B$ such that $\sigma_{j}=0$ (resp. $0<\sigma_{j}<1$, resp. $1<\sigma_{j}<2$ ). Then $B_{0}=B_{\text {wild }} \cup B_{\text {prim }}$, and it follows from (3) that

$$
\sum_{j \in B}\left\langle\sigma_{j}\right\rangle=1
$$

We call the special deformation datum $(\bar{Z}, \omega)$ normalized if $\bar{X}=\mathbb{P}_{k}^{1}$ and $\left\{\tau_{j} \mid j \in\right.$ $\left.B_{0}\right\}=\{0,1, \infty\}$.

Their connection to three point covers suggests that special deformation data are 'rigid' objects, i.e. there should not exist any nontrivial families of special deformation data. This is indeed true; a precise statement is formulated and proved in [Wew02], §5.4. See also $\$ 3$ below.

2.2.2. In this subsection, $\bar{X}$ will denote a smooth projective curve over $k$ of genus 0 , together with a distinguished closed point $\bar{\infty} \in \bar{X}$. We also fix a finite group $G$. 
Definition 2.9. A $G$-tail cover is a (not necessarily connected) $G$-cover $\bar{f}: \bar{Y} \rightarrow \bar{X}$ such that the following hold.

(1) The cover $\bar{f}$ is wildly ramified above $\bar{\infty}$, of order $p m$ with $(p, m)=1$.

(2) The restriction of $\bar{f}$ to $\bar{X}-\{\bar{\infty}\}$ is tame and branched at most at one point. A pointed $G$-tail cover is a $G$-tail cover together with a point $\eta \in \bar{Y}$ above $\bar{\infty}$ and a subgroup $H$ of the inertia group $I_{\eta}$ such that $I_{\eta}=I_{\eta, 1} \rtimes H$. An (outer) automorphism of a pointed $G$-tail cover is an automorphism $\gamma: \bar{Y} \stackrel{\sim}{\rightarrow} \bar{Y}$ which normalizes the action of $G$ (and hence induces an automorphism of $\bar{X}$ ), centralizes the action of $H \subset G$ and fixes the point $\eta$. Such an automorphism is called inner if it centralizes the action of $G$.

Let $(\bar{f}: \bar{Y} \rightarrow \bar{X}, \eta, H)$ be a pointed $G$-tail cover. By condition (1), $I_{\eta, 1}$ is cyclic of order $p$ and $H$ cyclic of order prime-to- $p$. Let $m$ denote the order of $H$ and $h$ the conductor of $I_{\eta, 1}$. The pair $(m, h)$ is called the ramification type and the rational number $\sigma:=h / m$ the ramification invariant of $\bar{f}$. Note that $h>0$ and $(h, p)=1$. Moreover, $m \mid(p-1) h$, by the Hasse-Arf theorem (see [Ser68], IV §3).

Definition 2.10. A tail cover $\bar{f}: \bar{Y} \rightarrow \bar{X}$ is called special if the following two conditions hold.

(1) We have $\sigma<2$ and $\sigma \neq 1$.

(2) If $\sigma>1$, then the restriction of $\bar{f}$ to $\bar{X}-\{\bar{\infty}\}$ is étale.

If $\sigma<1$, then we say that the tail cover $\bar{f}$ is primitive; otherwise we say that it is new.

By Ray99, Proposition 1.1.6, the converse of condition (2) holds automatically: if $\sigma<1$ then there exists a unique point $\overline{0} \in \bar{X}-\{\bar{\infty}\}$ above which $\bar{f}$ is tamely ramified. The reason is this: if $\bar{f}$ were étale over $\bar{X}-\{\bar{\infty}\}$ and $\sigma<1$, then the Riemann-Hurwitz formula would produce a negative number for the genus of $\bar{Y}$.

It is clear from the results of $\$ 2.1$ that the tail covers obtained from the stable reduction of a three point Galois cover are special (provided that the reduction is not exceptional). We can therefore expect that a special tail cover $\bar{f}: \bar{Y} \rightarrow \bar{X}$ is 'rigid'. In fact, using the methods of [BM00], one can show that the curve $\bar{Y}$ does not admit any nontrivial $G$-equivariant deformations. What is more important for us is that all special $G$-tail covers with a given ramification type $(m, h)$ are 'locally isomorphic', in the following sense.

Definition 2.11. Let $\left(\bar{f}_{1}: \bar{Y}_{1} \rightarrow \bar{X}, \eta_{1}, H\right)$ and $\left(\bar{f}_{2}: \bar{Y}_{2} \rightarrow \bar{X}, \eta_{2}, H\right)$ be two pointed $G$-tail covers with the same subgroup $H \subset G$ and the same ramification type $(m, h)$. Let $\bar{X}_{\bar{\infty}}$ denote the generic point of the completion of $\bar{X}$ at $\bar{\infty}$, and set $\bar{Y}_{i, \bar{\infty}}:=\bar{Y}_{i} \times_{\bar{X}} \bar{X}_{\bar{\infty}}$. A local isomorphism between $\bar{f}_{1}$ and $\bar{f}_{2}$ is a $G$-equivariant isomorphism

$$
\varphi_{\bar{\infty}}: \bar{Y}_{1, \bar{\infty}} \stackrel{\sim}{\longrightarrow} \bar{Y}_{2, \bar{\infty}}
$$

with the following properties.

(1) The automorphism $\psi_{\bar{\infty}}: \bar{X}_{\bar{\infty}} \stackrel{\sim}{\rightarrow} \bar{X}_{\bar{\infty}}$ induced by $\varphi_{\bar{\infty}}$ extends to a global automorphism $\psi: \bar{X} \stackrel{\sim}{\rightarrow} \bar{X}$ (which necessarily fixes $\bar{\infty}$ ).

(2) We have $\varphi_{\bar{\infty}}\left(\eta_{1}\right)=\eta_{2}$. Moreover, if $\bar{f}_{1}$ is ramified at $\overline{0}_{1} \in \bar{X}-\{\bar{\infty}\}$, then $\bar{f}_{2}$ is also ramified at some point $\overline{0}_{2} \in \bar{X}-\{\bar{\infty}\}$, and the automorphism $\psi$ in (1) can be chosen in such a way that $\psi\left(\overline{0}_{1}\right)=\overline{0}_{2}$. 
Lemma 2.12. All special pointed $G$-tail covers with given ramification type $(m, h)$ and subgroup $H \subset G$ are locally isomorphic.

Proof. This is a special case of the results of [Pri02], $\S 4$. For the convenience of the reader, we sketch a proof.

Let $(\bar{f}: \bar{Y} \rightarrow \bar{X}, \eta, H)$ be a special pointed $G$-tail cover, of ramification type $(m, h)$. We will show that $\bar{f}: \bar{Y} \rightarrow \bar{X}$ is uniquely determined, up to local isomorphism, by the datum $(G, H, m, h)$. First of all, by the definition of local isomorphism it suffices to look at tail covers $\bar{f}$ which are totally ramified above $\bar{\infty}$. In other words, we may assume that $G=I \rtimes H$, where $I \subset G$ is cyclic of order $p$ and $H$ is cyclic of order $m$. Second, we shall assume that the tail cover $\bar{f}$ is new. Hence we can write $h=m+a$, with $0<a<m$. Note that $m \mid a(p-1)$ and that $a$ is prime to $p$. We will indicate later how to modify the proof in the case that $\bar{f}$ is primitive.

Let $x$ be a global coordinate for $\bar{X} \cong \mathbb{P}_{k}^{1}$ such that $\bar{X}_{\bar{\infty}}=\operatorname{Spec} k\left(\left(x^{-1}\right)\right)$. By elementary Galois theory, the localized cover $\bar{Y}_{\bar{\infty}} \rightarrow \bar{X}_{\bar{\infty}}$ is given by two equations

$$
z^{m}=x, \quad y^{p}-y=z^{a}\left(b_{0} x+b_{1}+b_{2} x^{-1}+\ldots\right),
$$

with $b_{i} \in k$ and $b_{0} \neq 0$. The action of $G$ on $\bar{Y}_{\bar{\infty}}$ is given as follows. We may choose a generator $\alpha$ of $I \cong \mathbb{Z} / p$ such that $\alpha^{*} z=z$ and $\alpha^{*} y=y+1$. Furthermore, for any $\beta \in H$ we have $\beta^{*} z=\psi(\beta) \cdot z$ and $\beta^{*} y=\chi(\beta) \cdot y$, where $\psi: H \rightarrow \overline{\mathbb{F}}_{p}^{\times}$is a character of order $m$ such that $\chi=\psi^{a}$. See, e.g., [Pri02, Lemma 1.4.1.

A local isomorphism amounts to a change of coordinates

$$
x \mapsto c(x+d), \quad z \mapsto c^{1 / m} z\left(1+d x^{-1}\right)^{1 / m}, \quad y \mapsto y+g,
$$

where $c, d \in k, c \neq 0$ and $g=z^{a}\left(e_{1} x^{-1}+e_{2} x^{-2}+\ldots\right) \in k\left[\left[z^{-1}\right]\right]$. An easy computation shows that for a suitable choice of the constants $c$ and $d$, and after transforming the equations (10) by the change of coordinate (11), we may assume that $b_{0}=1$ and $b_{1}=0$. It is also easy to see that we may choose $e_{1}, e_{2}, \ldots$ in such a way that

$$
g^{p}-g=-z^{a}\left(b_{2} x^{-1}+b_{3} x^{-2}+\ldots\right)
$$

(here we use that $m \mid a(p-1)$ ). With this choice, equations (10) become

$$
z^{m}=x, \quad y^{p}-y=z^{h},
$$

which visibly depends only on the ramification type $(m, h)$. This proves the lemma for a new tail cover $\bar{f}$. The proof for a primitive tail cover is similar. The main difference is that we are only allowed to do a homothety $x \mapsto c x$ instead of a general affine transformation $x \mapsto c(x+d)$. However, since $0<h<m$, the proof goes through.

2.2.3. As before, $k$ denotes an algebraically closed field and $G$ a finite group. Suppose we are given the following data.

- A subgroup $G_{0} \subset G$, of the form $G_{0}=I_{0} \rtimes H_{0}$, where $I_{0} \subset G_{0}$ is cyclic of order $p$ and $H_{0} \subset G_{0}$ is of prime-to- $p$ order. Let $\chi_{0}: H_{0} \rightarrow \mathbb{F}_{p}^{\times}$denote the character defined by the rule $\beta \alpha \beta^{-1}=\alpha^{\chi_{0}(\beta)}$, where $\alpha \in I_{0}, \beta \in H_{0}$.

- A normalized special deformation datum $\left(\bar{Z}_{0}, \omega_{0}\right)$ over $\bar{X}_{0}:=\mathbb{P}_{k}^{1}$ of type $\left(H_{0}, \chi_{0}\right)$. Let $\left(\tau_{j}\right)_{j \in B}$ denote the set of critical points, $m_{j}, h_{j}$ and $\sigma_{j}$ the invariants attached to $\left(\bar{Z}_{0}, \omega_{0}, \tau_{j}\right)$.

- For all $j \in B-B_{\text {wild }}$ a point $\xi_{j} \in \bar{Z}_{0}$ above $\tau_{j} \in \bar{X}_{0}$. 
- For all $j \in B-B_{\text {wild }}$ a subgroup $G_{j} \subset G$ and a connected pointed $G_{j}$-tail cover $\left(\bar{f}_{j}: \bar{Y}_{j} \rightarrow \bar{X}_{j}, \eta_{j}, H_{j}\right)$.

By abuse of notation, we shall write $\left(\bar{Z}_{0}, \omega_{0} ; \bar{f}_{j}\right)$ to denote all of the data introduced above.

Definition 2.13. The datum $\left(\bar{Z}_{0}, \omega_{0} ; \bar{f}_{j}\right)$ is called a special $G$-deformation datum if the following conditions hold.

(1) The group $G$ is generated by its subgroups $G_{0}$ and $G_{j}$.

(2) The tail cover $\bar{f}_{j}: \bar{Y}_{j} \rightarrow \bar{X}_{j}$ has ramification of type $\left(m_{j}, h_{j}\right)$. Moreover,

$$
I_{\eta_{j}}=I_{0} \rtimes H_{j}, \quad \text { and } H_{j}=H_{0} \cap I_{\eta_{j}} .
$$

It is clear from the results of 2.1 that the stable reduction of a three point cover with mild reduction gives rise to a special $G$-deformation datum. To make this statement more precise, we introduce the following terminology.

Definition 2.14. A stable $G$-map over a scheme $S$ is a homomorphism $f: Y \rightarrow X$ of marked semistable curves over $S$ together with an $S$-linear action of $G$ on $Y$ such that $Y$ is stably marked and $f$ commutes with the $G$-action. Two stable $G$-maps $f_{i}: Y_{i} \rightarrow X_{i}$ are said to be isomorphic if there exist isomorphisms $\varphi: Y_{1} \stackrel{\sim}{\rightarrow} Y_{2}$ and $\psi: X_{1} \rightarrow X_{2}$ of marked curves such that $f_{2} \circ \varphi=\psi \circ f_{1}$ and such that $\varphi$ is $G$-equivariant.

If $f: Y \rightarrow X$ is a $G$-cover over $\bar{K}$, as in $\$ 1.1$ then we may consider the stable reduction $\bar{f}: \bar{Y} \rightarrow \bar{X}$ of $f$ as a stable $G$-map over $k$.

Fix a special $G$-deformation datum $\left(\bar{Z}_{0}, \omega_{0} ; \bar{Y}_{j}\right)$. We will show that $\left(\bar{Z}_{0}, \omega_{0} ; \bar{Y}_{j}\right)$ gives rise to a stable $G$-map $\bar{f}: \bar{Y} \rightarrow \bar{X}$ over $k$. Define

$$
\bar{X}:=\left(\bar{X}_{0} \amalg \coprod_{j} \bar{X}_{j}\right) / \sim .
$$

Here the equivalence relation ' $\sim$ ' identifies the critical point $\tau_{j} \in \bar{X}_{0}$ with the distinguished point $\bar{\infty}_{j} \in \bar{X}_{j}$. The topological space $\bar{X}$ carries a unique structure of a semistable $k$-curve such that $\bar{X}_{0}$ and $\bar{X}_{j}$ are the irreducible components of $\bar{X}$. We define marked points $\bar{x}_{j}$ on $\bar{X}$ (indexed by the set $B_{0}=B_{\text {prim }} \cup B_{\text {wild }}$ ), as follows. For $j \in B_{\text {prim }}$ we let $\bar{x}_{j} \in \bar{X}_{j}$ be the unique tame branch point of the tail cover $\bar{f}_{j}$ (see the remark after Definition 2.10). For $j \in B_{\text {wild }}$ we set $\bar{x}_{j}:=\tau_{j}$.

To define the curve $\bar{Y}$, let $\bar{Y}_{0}$ be the smooth projective curve with function field $k\left(\bar{Z}_{0}\right)^{1 / p}$. The action of $H_{0}$ on $\bar{Z}_{0}$ extends uniquely to an action on $\bar{Y}_{0}$. We let $G_{0}$ act on $\bar{Y}_{0}$ via the quotient map $G_{0} \rightarrow H_{0}$. Furthermore, we define

$$
\bar{Y}:=\left(\operatorname{Ind}_{G_{0}}^{G}\left(\bar{Y}_{0}\right) \amalg \coprod_{j} \operatorname{Ind}_{G_{j}}^{G}\left(\bar{Y}_{j}\right)\right) / \sim .
$$

Here the equivalence relation ' $\sim$ ' is defined as follows: for $\eta^{\prime} \in \operatorname{Ind}_{G_{0}}^{G}\left(\bar{Y}_{0}\right)$ and $\eta^{\prime \prime} \in \operatorname{Ind}_{G_{j}}^{G}\left(\bar{Y}_{j}\right)$ we have $\eta^{\prime} \sim \eta^{\prime \prime}$ if and only if there exists an element $\alpha \in G$ such that $\alpha\left(\eta^{\prime}\right) \in \bar{Y}_{0}$ lies above $\xi_{j} \in \bar{Z}_{0}$ and $\alpha\left(\eta^{\prime \prime}\right)=\eta_{j} \in \bar{Y}_{j}$. (This is an equivalence relation because of condition (2) of Definition [2.13) Again, $\bar{Y}$ carries a canonical structure of a semistable curve over $k$. It follows from condition (1) of Definition 2.13 that $\bar{Y}$ is connected. Let $\bar{f}: \bar{Y} \rightarrow \bar{X}$ be the unique map which commutes with the natural $G$-action on $\bar{Y}$ and whose restriction to $\bar{Y}_{0}$ (resp. to $\bar{Y}_{j}$ ) is equal to $\bar{f}_{0}$ (resp. to $\bar{f}_{j}$ ). The marked points of $\bar{Y}$ are, by definition, those which lie over a marked point of $\bar{X}$. Thus, a point on $\bar{Y}$ is marked either if it is the $G$-translate of 
a ramification point of a tail cover $\bar{f}_{j}$ or if it is a $G$-translate of a point on $\bar{Y}_{0}$ which lies above a wild critical point. One checks that $\bar{f}: \bar{Y} \rightarrow \bar{X}$ is a stable $G$-map. We call $\bar{X}_{0}=\mathbb{P}_{k}^{1}$ the original component of $\bar{f}$.

Definition 2.15. A stable $G$-map $\bar{f}: \bar{Y} \rightarrow \bar{X}$ over $k$ is called special if there exists a special $G$-deformation datum $\left(\bar{Z}_{0}, \omega_{0} ; \bar{Y}_{j}\right)$ such that $\bar{f}$ is isomorphic to the stable $G$-map constructed above.

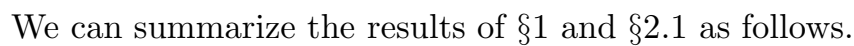

Proposition 2.16. Let $f: Y \rightarrow X=\mathbb{P}_{\bar{K}}^{1}$ be a G-Galois cover branched at 0,1 and $\infty$, with mild reduction. Then the stable reduction of $f$ is either exceptional or special.

In 4.1 we will prove the converse of this proposition: every special $G$-map arises as the stable reduction of a three point cover $f: Y \rightarrow X=\mathbb{P}_{\bar{K}}^{1}$ with mild reduction.

2.2.4. Let us fix a special $G$-deformation datum $\left(\bar{Z}_{0}, \omega_{0} ; \bar{Y}_{j}\right)$ and let $\bar{f}: \bar{Y} \rightarrow \bar{X}$ be the associated special $G$-map. Let us denote by $\operatorname{Aut}_{G}^{0}(\bar{f})$ the group of automorphisms of the special $G$-map $\bar{f}$ which induce the identity on the original component $\bar{X}_{0}$. If $\bar{f}$ is the stable reduction of a three point $G$-cover $f: Y \rightarrow X$, then the image of the inner monodromy action $\bar{\kappa}^{\text {in }}$ associated to $f$ is contained in $\operatorname{Aut}_{G}^{0}(\bar{f}) / C_{G}$. We are interested in a description of $\operatorname{Aut}_{G}^{0}(\bar{f})$ in terms of the special deformation datum $\left(\bar{Z}_{0}, \omega_{0} ; \bar{Y}_{j}\right)$.

Lemma 2.17. There is a natural bijection between the group $\operatorname{Aut}_{G}^{0}(\bar{f})$ and the set of tuples $\left(\gamma_{0} ; \gamma_{j}\right)$, where $\gamma_{0} \in G$ and $\gamma_{j}: \bar{Y}_{j} \stackrel{\sim}{\rightarrow} \bar{Y}_{j}$ is an (outer) automorphism of the pointed $G_{j}$-tail cover $\left(\bar{f}_{j}, \eta_{j}, H_{j}\right)$ (see Definition [2.9) such that the following hold.

(1) The element $\gamma_{0} \in G$ normalizes $I_{0}$ and centralizes $H_{0}$.

(2) The equation

$$
\gamma_{0}^{-1} \circ \alpha \circ \gamma_{0}=\gamma_{j} \circ \alpha \circ \gamma_{j}^{-1}
$$

holds for all $\alpha \in G_{j}$ and $j \notin B_{\text {wild }}$.

Proof. Let $\gamma: \bar{Y} \stackrel{\sim}{\rightarrow} \bar{Y}$ be an element of $\operatorname{Aut}_{G}^{0}(\bar{f})$. It is clear that we can find an element $\gamma_{0} \in G$ such that $\gamma_{0}^{-1} \circ \gamma$ induces the identity on $\bar{Y}_{0}$. It is also clear that $\gamma_{0}$ normalizes $G_{0}$ and that conjugation by $\gamma_{0}$ induces the identity on $G_{0} / I_{0} \cong H_{0}$. Since the order of $H_{0}$ is prime to $p$, the Schur-Zassenhaus theorem implies that the subgroup $\gamma_{0} H_{0} \gamma_{0}^{-1} \subset G_{0}$ is conjugate to $H_{0}$ by an element of $G_{0}$. Using the fact that the action of $H_{0}$ on $I_{0}$ is irreducible, one shows that there exists a unique element $\alpha \in I_{0}$ such that

$$
\gamma_{0} \circ \beta \circ \gamma_{0}^{-1}=\alpha^{-1} \circ \beta \circ \alpha
$$

for all $\beta \in H_{0}$. After replacing $\gamma_{0}$ by $\alpha \circ \gamma_{0}$, we may thus assume that $\gamma_{0}$ centralizes $H_{0}$. For all $j \notin B_{\text {wild }}$, set $\gamma_{j}:=\left.\gamma_{0}^{-1} \circ \gamma\right|_{\bar{Y}_{j}}$. One easily checks that $\gamma_{j}$ is an (outer) automorphism of the pointed $G_{j}$-tail cover $\left(\bar{f}_{j}, \eta_{j}, H_{j}\right)$ and that the tuple $\left(\gamma_{0} ; \gamma_{j}\right)$ satisfies conditions (1) and (2).

Conversely, given a tuple $\left(\gamma_{0} ; \gamma_{j}\right)$ such that (1) and (2) hold, we define an automorphism $\gamma: \bar{Y} \stackrel{\sim}{\rightarrow} \bar{Y}$ of $\bar{f}$, as follows. If $y$ is a point on $\bar{Y}_{0}$ and $\alpha$ is an element of $G$, we set

$$
\gamma(\alpha(y)):=\alpha \circ \gamma_{0}(y) .
$$


This is well defined because of condition (1). If $y$ is a point on $\bar{Y}_{j}$ and $\alpha \in G$, we set

$$
\gamma(\alpha(y)):=\alpha \circ \gamma_{0} \circ \gamma_{j}(y) .
$$

This is well defined because $\gamma \circ \gamma_{j}$ commutes with $G_{j}$, by condition (2). Moreover, the two definitions respect the equivalence relation ' $\sim$ ' used in the definition of $\bar{Y}$ in $\{2.2 .3$. Hence we obtain an automorphism $\gamma: \bar{Y} \stackrel{\sim}{\rightarrow} \bar{Y}$, which is easily seen to be an element of $\operatorname{Aut}_{G}^{0}(\bar{f})$. This proves the lemma.

The association $\gamma \mapsto \gamma_{0}$ is a group homomorphism $\operatorname{Aut}_{G}^{0}(\bar{f}) \rightarrow G$. Let us denote by $n$ the order of the image of this homomorphism in $G / C_{G}$. Note that $n \mid(p-1)$. Let us denote by $\operatorname{Aut}_{G}\left(\bar{f}_{j}\right)$ the group of inner automorphisms of the pointed $G_{j}$-tail covers $\left(\bar{f}_{j}, \eta_{j}, H_{j}\right)$. The lemma shows that

$$
\left|\operatorname{Aut}_{G}^{0}(\bar{f})\right|=n \cdot\left|C_{G}\right| \cdot \prod_{j \notin B_{\text {wild }}}\left|\operatorname{Aut}_{G}\left(\bar{f}_{j}\right)\right| .
$$

Remark 2.18. Suppose that the character $\chi: H_{0} \rightarrow \mathbb{F}_{p}^{\times}$is injective. (In particular, $H_{0}$ is cyclic.) Then the integer $n$ defined above is a multiple of

$$
n^{\prime}:=\operatorname{gcd}_{j}\left(m_{j}\right) \text {. }
$$

In fact, let $\gamma$ be an element of $H_{0}$ which is contained in the stabilizer $H_{j}$ of $\xi_{j}$, for all $j \in B_{\text {prim }} \cup B_{\text {new }}$. Then the tuple $\left(\gamma_{0} ; \gamma_{j}\right)$ with $\gamma_{j}:=\left.\gamma_{0}^{-1}\right|_{\bar{Y}_{j}}$ represents an element of $\operatorname{Aut}_{G}^{0}(\bar{f})$ which does not lie in $C_{G}$ and which acts trivially on the vertical components of $\bar{Y}$.

\section{THE AUXILIARY COVER}

Let $(\bar{Z}, \omega)$ be a special deformation datum of type $(H, \chi)$. By a lift of $(\bar{Z}, \omega)$ we mean a Galois cover $f: Y \rightarrow X=\mathbb{P}^{1}$ with Galois group $G:=\mathbb{Z} / p \rtimes_{\chi} H$ such that the deformation datum occurring over the original component of the stable reduction of $f$ is equal to $(\bar{Z}, \omega)$. As we will see below, such a lift is uniquely determined by the deformation datum $(\bar{Z}, \omega)$ and the set of branch points $\left(x_{j}\right)$ of $f$ (which lift the critical points $\left(\tau_{j}\right)$ of $(\bar{Z}, \omega)$ ).

Following [Wew03, we say that the lift $f: Y \rightarrow X$ is special if the stable reduction of $f$ is as simple as possible, i.e. if the curve $\bar{X}$ is a comb. For instance, if $f: Y \rightarrow X$ is the auxiliary cover associated to a three point cover with mild reduction, then $f$ is special by Theorem 2.6 (note that $f$ is in general not a three point cover itself).

In Wew03 we showed that a lift $f$ of $(\bar{Z}, \omega)$ is special if each branch point of $f$ is 'sufficiently close' to a $K_{0}$-rational point. The main result of this section - Theorem 3.8 - says that the converse is true as well. This theorem will play a crucial role in \$4.

The proof of Theorem 3.8 relies on the results of Wew02, which concern the deformation theory of a certain curve with group scheme action associated to $(\bar{Z}, \omega)$. The connection is as follows. If $f: Y \rightarrow X$ is a lift of the deformation datum $(\bar{Z}, \omega)$, then there exist a certain natural $R$-model $f_{R}: Y_{R} \rightarrow X_{R}=\mathbb{P}_{R}^{1}$ (which is not the stable model) such that the $G$-action on $Y$ extends to an action of the group scheme $\mathcal{G}:=\boldsymbol{\mu}_{p} \rtimes H$ on $Y_{R}$. The special fiber $\bar{Y}$ of $Y_{R}$ is a singular curve with an action of $\mathcal{G}$; it depends on $(\bar{Z}, \omega)$ but not on the lift $f: Y \rightarrow X$. Therefore, a lift of $(\bar{Z}, \omega)$ is the same thing as an equivariant deformation of $\bar{Y}$. 


\subsection{The $\mathcal{G}$-cover associated to a multiplicative deformation datum.}

3.1.1. Throughout this section, we fix an odd prime $p$ and an algebraically closed field $k$ of characteristic $p$. We denote by $W(k)$ the ring of Witt vectors over $k$.

Let $\bar{X}$ be a smooth projective curve over $k$ and let $(\bar{Z}, \omega)$ be a multiplicative deformation datum over $\bar{X}$ of type $(H, \chi)$, see Definition 1.5 In this section we show that $(\bar{Z}, \omega)$ corresponds naturally to a (not necessarily smooth) curve $\bar{Y}$ over $k$ together with an action of a certain group scheme $\mathcal{G}$ such that $\bar{X}=\bar{Y} / \mathcal{G}$.

We define $\mathcal{G}$ as a group scheme over $W(k)$, as follows. If $R$ is a $W(k)$-algebra, an element of $\mathcal{G}(R)$ is given by a tuple $(\zeta, \beta)$, where $\zeta \in R$ satisfies $\zeta^{p}=1$ and $\beta \in H$. Multiplication is defined as follows:

$$
\left(\zeta_{1}, \beta_{1}\right) \cdot\left(\zeta_{2}, \beta_{2}\right):=\left(\zeta_{1} \zeta_{2}^{\chi\left(\beta_{1}\right)}, \beta_{1} \beta_{2}\right) .
$$

In other words, $\mathcal{G}$ is the semidirect product $\boldsymbol{\mu}_{p} \rtimes H$ determined by the action of $H$ on $\boldsymbol{\mu}_{p}$ via $\chi$. If $R$ is a $W(k)$-algebra and $Y$ a scheme over $R$, we say that $\mathcal{G}$ acts on $Y$ if we are given an $R$-linear action of $\mathcal{G} \otimes R$ on $Y$.

Let $\left(\tau_{j}\right)_{j \in B}$ be the set of critical points for the deformation datum $(\bar{Z}, \omega)$. For $j \in B$, let $m_{j}, h_{j}$ be the invariants attached to $\left(\bar{Z}, \omega, \tau_{j}\right)$, see $\$ 1.3$. A critical point $\tau_{j}$ is called a tame (resp. wild) branch point if $m_{j}>1$ (resp. if $h_{j}=0$ ). We denote by $B_{\text {tame }}$ and $B_{\text {wild }}$ the corresponding subsets of $B$ and set $B_{\text {ram }}:=B_{\text {tame }} \cup B_{\text {wild }}$. In general we have $B_{\text {tame }} \cap B_{\text {wild }} \neq \emptyset$ and $B_{\text {ram }} \neq B$.

Let $\bar{U} \subset \bar{X}$ be the complement of the wild branch points and let $\bar{V} \subset \bar{Z}$ be its inverse image. Then $\omega$ is a regular and logarithmic differential form on $\bar{V}$. Let $\bar{W} \rightarrow \bar{V}$ be the corresponding $\boldsymbol{\mu}_{p}$-torsor, see, e.g., Mil80, p. 129. The definition of $\bar{W}$ is roughly the following. Locally on $\bar{V}$ we can write $\omega=\mathrm{d} u / u$ for a unit $u$. Then $y^{p}=u$ is a local equation for the $\boldsymbol{\mu}_{p}$-torsor $\bar{W} \rightarrow \bar{V}$.

Proposition 3.1. Let $\bar{Y}$ be the projective compactification of $\bar{W}$ such that the complement $\bar{Y}-\bar{W}$ is contained in the smooth locus of $\bar{Y}$. The action of $\boldsymbol{\mu}_{p}$ on $\bar{W}$ extends uniquely to an action of $\mathcal{G}$ on $\bar{Y}$ such that $\bar{Z}=\bar{Y} / \boldsymbol{\mu}_{p}$ and $\bar{X}=\bar{Y} / \mathcal{G}$.

Proof. Let $\lambda: \boldsymbol{\mu}_{p} \times \bar{W} \rightarrow \bar{W}$ denote the action of $\boldsymbol{\mu}_{p}$ on $\bar{W}$. We have to show that $\lambda$ extends to all of $\bar{Y}$. Let $\eta \in \bar{Y}-\bar{W}$ be a point in the complement of $\bar{W}$ and let $\xi \in \bar{Z}$ be its image. By construction, the point $\xi$ lies above a wild critical point $\tau_{j}$, so $\omega$ has a simple pole at $\xi$. Write $\omega=a \mathrm{~d} u / u$, where $u$ is a regular function in a neighborhood of $\xi$ with a simple zero at $\xi$ and $a \in \mathbb{F}_{p}^{\times}$is the residue of $\omega$ at $\xi$. By definition of $\bar{Y}$ there exists a rational function $y$ on $\bar{Y}$ such that $y^{p}=u$ and $\lambda^{*} y=\zeta^{b} \otimes y$, for some $b \in \mathbb{F}_{p}^{\times}$. It follows also from the definition of $\bar{Y}$ that $\eta$ is a smooth point of $\bar{Y}$. Therefore, the integral equation $y^{p}=u$ shows that $y \in \mathcal{O}_{\bar{Y}, \eta}$. Moreover, since $u$ has a simple zero at $\xi$, we have $\mathcal{O}_{\bar{Y}, \eta}=\mathcal{O}_{\bar{Z}, \xi}\left[y \mid y^{p}=u\right]$. This shows that the action $\lambda$ extends to $\bar{Y}$. It is obvious that this extension is unique.

It remains to show that $\lambda$ extends to an action of $\mathcal{G}$ on $\bar{Y}$ which induces the canonical action of $H$ on $\bar{Z}$. But this is clear from the rule $\beta^{*} \omega=\chi(\beta) \cdot \omega$ and the definition of $\bar{Y}$.

The map $\bar{Y} \rightarrow \bar{X}$ together with the action of $\mathcal{G}$ on $\bar{Y}$ will be called the $\mathcal{G}$-cover associated to $(\bar{Z}, \omega)$. Note that the curve $\bar{Y}$ is integral and has isolated, 'cusp-like' singularities precisely above the critical points which are not wild branch points, see Ray90. 
3.1.2. Let $\mathfrak{C}_{k}$ be the category of Noetherian Artinian local $W(k)$-algebras with residue field $k$. An equivariant deformation of $\bar{Y}$ over $R \in \mathfrak{C}_{k}$ is a deformation $Y$ of the curve $\bar{Y}$ over $R$ together with an action of $\mathcal{G}$ on $Y$ such that the natural isomorphism $\bar{Y} \cong Y \otimes_{R} k$ is $\mathcal{G}$-equivariant. Let $\operatorname{Def}(\bar{Y}, \mathcal{G})$ denote the functor which associates to a ring $R \in \mathfrak{C}_{k}$ the set of isomorphism classes of equivariant deformations of $\bar{Y}$ over $R$.

Given an equivariant deformation $Y_{R}$ of $\bar{Y}$, we denote by $Z_{R}:=Y_{R} / \boldsymbol{\mu}_{p}$ and $X_{R}:=Y_{R} / \mathcal{G}$ the quotient schemes by $\boldsymbol{\mu}_{p}$ and $\mathcal{G}$, respectively. The $R$-curve $X_{R}$ (resp. $Z_{R}$ ) is a smooth deformation of the curve $\bar{X}$ (resp. $\bar{Z}$ ). The association $Y_{R} \mapsto$ $X_{R}$ gives rise to a morphism of deformation functors $\operatorname{Def}(\bar{Y}, \mathcal{G}) \rightarrow \operatorname{Def}(\bar{X})$. Here $\operatorname{Def}(\bar{X})$ denotes the functor which classifies deformations of $\bar{X}$. (One has to check that formation of the quotient commutes with base change. This is easy, using the description of $\boldsymbol{\mu}_{p}$-covers by Kummer equations.) Let us write $\operatorname{Def}\left(\bar{X} ; \tau_{j} \mid j \in B_{\text {ram }}\right)$ for the functor which classifies deformations of the pointed curve $\left(\bar{X} ; \tau_{j} \mid j \in B_{\text {ram }}\right)$. We claim that the morphism $\operatorname{Def}(\bar{Y}, \mathcal{G}) \rightarrow \operatorname{Def}(\bar{X})$ factors in a natural way over the forgetful morphism $\operatorname{Def}\left(\bar{X} ; \tau_{j} \mid j \in B_{\text {ram }}\right) \rightarrow \operatorname{Def}(\bar{X})$, thus inducing a morphism

$$
\operatorname{Def}(\bar{Y}, \mathcal{G}) \longrightarrow \operatorname{Def}\left(\bar{X} ; \tau_{j} \mid j \in B_{\mathrm{ram}}\right) \text {. }
$$

Let $Y_{R}$ be an equivariant deformation of $\bar{Y}$ and set $X_{R}:=Y_{R} / \mathcal{G}, Z_{R}:=Y_{R} / \boldsymbol{\mu}_{p}$. To prove the claim we have to endow, in a functorial way, the curve $X_{R}$ with sections $\tau_{j, R}$ : Spec $R \rightarrow X_{R}$ lifting the branch points $\tau_{j}$, for all $j \in B_{\text {ram }}$. It is clear that the natural map $Z_{R} \rightarrow X_{R}$ is a tame $H$-Galois cover lifting $\bar{Z} \rightarrow \bar{X}$. By the theory of tame ramification, the branch divisor of $Z_{R} \rightarrow X_{R}$ consists of sections $\tau_{j, R}:$ Spec $R \rightarrow X_{R}$ lifting the tame branch points $\tau_{j} \in \bar{X}$, for all $j \in B_{\text {tame }}$. Now let $j \in B_{\text {wild }}$ and choose a point $\xi \in \bar{Z}$ above $\tau_{j}$. We can write $\omega=\mathrm{d} u / u$ with $\operatorname{ord}_{\xi}(u)=1$. By construction, the restriction of $\bar{Y} \rightarrow \bar{Z}$ to a neighborhood of $\xi$ is a $\boldsymbol{\mu}_{p}$-cover given by the Kummer equation $y^{p}=u$. Hence the deformation $Y_{R} \rightarrow Z_{R}$ is, locally around $\xi$, still a $\boldsymbol{\mu}_{p}$-cover given by a Kummer equation $y^{p}=u_{R}$, where $u_{R}$ lifts $u$. The equation $u_{R}=0$ defines a section $\xi_{R}$ : Spec $R \rightarrow Z_{R}$ lifting $\xi$. We define $\tau_{j, R}$ : Spec $R \rightarrow X_{R}$ as the image of $\xi_{R}$. Using the $H$-action, it is easy to see that our two definitions of $\tau_{j, R}$ agree if $j \in B_{\text {tame }} \cap B_{\text {wild }}$. This proves the claim.

We say that $Y_{R} \rightarrow X_{R}$ is a deformation of the $\mathcal{G}$-cover $\bar{Y} \rightarrow \bar{X}$, and we call the tuple $\left(\tau_{j, R}\right)_{j \in B_{\text {ram }}}$ the branch locus of $Y_{R} \rightarrow X_{R}$. It is clear that a deformation of the $\mathcal{G}$-cover $\bar{Y} \rightarrow \bar{X}$ is the same thing as an equivariant deformation of $\bar{Y}$.

\subsection{The case of a special deformation datum.}

3.2.1. We continue with the notation of $\$ 3.1$ In addition, we assume from now on that the deformation datum $(\bar{Z}, \omega)$ is special, see Definition 2.7. We use the notation $B_{\text {prim }}, B_{\text {new }}$ and $B_{0}$ as in $\$ 2.2 .1$. It follows immediately from the definitions that we have $B_{\text {tame }}=B_{\text {prim }} \cup B_{\text {new }}$ and $B_{\text {wild }}=B_{0}-B_{\text {prim }}$. In particular, we have $B_{\text {tame }} \cap B_{\text {wild }}=\emptyset$ and $B=B_{\text {ram }}$. Furthermore, we assume that the special deformation datum $(\bar{Z}, \omega)$ is normalized, i.e. we have $\bar{X}=\mathbb{P}_{k}^{1}$ and $\left\{\tau_{j} \mid j \in B_{0}\right\}=$ $\{0,1, \infty\}$.

Let $Y_{R} \rightarrow X_{R}$ be a deformation of the $\mathcal{G}$-cover $\bar{Y} \rightarrow \bar{X}$ with branch locus $\left(\tau_{j, R}\right)_{j \in B}$. There exists a unique isomorphism $X_{R} \cong \mathbb{P}_{R}^{1}$ which reduces to the identity on $\bar{X}=\mathbb{P}_{k}^{1}$ and is such that $\left\{\tau_{j, R} \mid j \in B_{0}\right\} \stackrel{R}{=}\{0,1, \infty\}$. Hence we may, and will, identify $X_{R}$ with $\mathbb{P}_{R}^{1}$ and the sections $\tau_{j, R}$, for $j \in B_{\text {new }}$, with the corresponding elements of $R$. A $B$-tuple $\left(\tau_{j, R}\right)$ of $R$-points of $X_{R}=\mathbb{P}_{R}^{1}$ such that 
$\tau_{j, R}$ lifts $\tau_{j}$ for all $j \in B$ and $\left\{\tau_{j, R} \mid j \in B_{0}\right\}=\{0,1, \infty\}$ is called a normalized $B$-tuple over $R$.

Proposition 3.2. The morphism of deformation functors

$$
\operatorname{Def}(\bar{Y}, \mathcal{G}) \longrightarrow \operatorname{Def}\left(\bar{X} ; \tau_{j}\right)
$$

is an isomorphism. In other words, given $R \in \mathfrak{C}_{k}$ and a normalized B-tuple $\left(\tau_{j, R}\right)$ over $R$, there exists a deformation $Y_{R} \rightarrow X_{R}=\mathbb{P}_{R}^{1}$ of the $\mathcal{G}$-cover $\bar{Y} \rightarrow \bar{X}=\mathbb{P}_{k}^{1}$ with branch locus $\left(\tau_{j, R}\right)$, unique up to a unique isomorphism.

In particular, the deformation functor $\operatorname{Def}(\bar{Y}, \mathcal{G})$ is formally smooth.

Proof. This is a special case of Wew02, Theorem 5.7.

For $j \in B_{\text {new }}$, let $T_{j}$ be an indeterminate; set $\tilde{R}:=W(k)\left[\left[T_{j} \mid j \in B_{\text {new }}\right]\right]$. For $j \in B_{\text {new }}$, let $\left[\tau_{j}\right] \in W(k)$ denote the Teichmüller lift of $\tau_{j}$ (in fact, any lift of $\tau_{j}$ to an element of $W(k)$ would do as well). Let $\left(\tilde{\tau}_{j}\right)$ be the normalized $B$-tuple over $\tilde{R}$ which has $\tilde{\tau}_{j}=\left[\tau_{j}\right]+T_{j}$ for $j \in B_{\text {new }}$. Let $\mathcal{X}$ denote the formal completion along the special fiber of the projective line over $\tilde{R}$. It is clear that for any $R \in \mathfrak{C}_{k}$ there is a one-to-one correspondence between local $W(k)$-algebra homomorphisms $\tilde{R} \rightarrow R$ and normalized $B$-tuples over $R$. Therefore, $\left(\mathcal{X} ; \tilde{\tau}_{j}\right)$ is the universal formal deformation of the pointed curve $\left(\bar{X} ; \tau_{j}\right)$. Hence Proposition 3.2 implies

Corollary 3.3. Let $\mathcal{Y} \rightarrow \mathcal{X}$ be the (unique) formal deformation of the $\mathcal{G}$-cover $\bar{Y} \rightarrow$ $\bar{X}$ with branch locus $\left(\tilde{\tau}_{j}\right)$. Then $\mathcal{Y}$ is a universal formal equivariant deformation of $\bar{Y}$.

3.2.2. For $j \in B$, we denote by $\hat{X}_{j}$ the completion of $\bar{X}$ at $\tau_{j}$ and set $\hat{Y}_{j}:=\bar{Y} \times_{\bar{X}} \hat{X}_{j}$. The action of $\mathcal{G}$ on $\bar{Y}$ induces an action on $\hat{Y}_{j}$ such that $\hat{X}_{j}=\hat{Y}_{j} / \mathcal{G}$. Given a deformation $Y_{R} \rightarrow X_{R}$ of the $\mathcal{G}$-cover $\bar{Y} \rightarrow \bar{X}$, we denote by $\hat{X}_{j, R}$ the completion of $X_{R}$ at $\tau_{j}$ and set $\hat{Y}_{j, R}:=\hat{X}_{j} \times_{X_{R}} \hat{X}_{j, R}$. Then $\hat{Y}_{j, R}$ is a $\mathcal{G}$-equivariant deformation of $\hat{Y}_{j}$. The association $Y_{R} \mapsto\left(\hat{Y}_{j, R}\right)_{j \in B}$ defines a morphism of deformation functors

$$
\Phi: \operatorname{Def}(\bar{Y}, \mathcal{G}) \longrightarrow \prod_{j \in B} \operatorname{Def}\left(\hat{Y}_{j}, \mathcal{G}\right) .
$$

Here $\operatorname{Def}\left(\hat{Y}_{j}, \mathcal{G}\right)$ denotes the deformation functor which classifies $\mathcal{G}$-equivariant formal deformations of $\hat{Y}_{j}$. Following [BM00, we call $\Phi$ the local-global morphism.

Proposition 3.4. $\quad(1)$ The functor $\operatorname{Def}\left(\hat{Y}_{j}, \mathcal{G}\right)$ admits a miniversal deformation over the ring

$$
\tilde{R}_{j}:= \begin{cases}W(k), & \text { for } j \in B_{0}, \\ W(k)\left[\left[t_{j}\right]\right], & \text { for } j \in B_{\mathrm{new}}\end{cases}
$$

Here $t_{j}$ is an indeterminate.

(2) The local-global morphism $\Phi$ is an isomorphism. Therefore,

$$
\tilde{R} \cong \widehat{\bigotimes}_{W(k)} \tilde{R}_{j}=W(k)\left[\left[t_{j} \mid j \in B_{\text {new }}\right]\right] .
$$

(3) For every $j \in B_{\text {new }}$ there exists a unit $w_{j} \in \tilde{R}$ such that

$$
t_{j} \equiv w_{j} T_{j} \quad(\bmod p) .
$$

Proof. Assertions (1) and (2) are a special case of [Wew02], Theorem 5.11. Assertion (3) corresponds to [Wew02], Proposition 5.14. 
3.2.3. Let $\mathcal{Y} \rightarrow \mathcal{X}$ be the universal deformation over $\tilde{R}$ of the $\mathcal{G}$-cover $\bar{Y} \rightarrow \bar{X}$, as defined in 3.2.1 Fix an index $j \in B_{\text {new }}$. We denote by $\hat{\mathcal{X}}_{j}$ the completion of $\mathcal{X}$ at $\tau_{j}$ and set $\hat{\mathcal{Y}}_{j}:=\mathcal{Y} \times{ }_{\mathcal{X}} \hat{\mathcal{X}}_{j}$. Being an equivariant deformation of $\hat{Y}_{j}$ over $\tilde{R}, \hat{\mathcal{Y}}_{j}$ is induced from the miniversal deformation of Proposition 3.4 via a homomorphism of $W(k)$ algebras $\varphi_{j}: \tilde{R}_{j} \rightarrow \tilde{R}$. The homomorphism $\varphi_{j}$ is unique up to an automorphism of $W(k)$-algebra $\tilde{R}_{j}$. Since $\varphi_{j}$ is injective by Proposition 3.4 (2), we may identify the $\operatorname{ring} \tilde{R}_{j}$ with the image of $\varphi_{j}$. By Proposition 3.4 (1) the ring $\tilde{R}_{j}$ is a formal power series over $W(k)$ in one variable $t_{j}$. It is clear that assertion (3) of Proposition 3.4 does not depend on the choice of the parameter $t_{j}$ as long as $\tilde{R}_{j} \cong W(k)\left[\left[t_{j}\right]\right]$.

The quotient $\mathcal{Z}:=\mathcal{Y} / \boldsymbol{\mu}_{p}$ is a formal $H$-equivariant deformation of $\bar{Z}$. Let us choose a point $\xi \in \bar{Z}$ above $\tau_{j}$ and denote by $\hat{\mathcal{Z}}_{j}$ the completion of $\mathcal{Z}$ at $\xi_{j}$. Since $\mathcal{Z}$ is formally smooth over $\tilde{R}$, we have $\hat{\mathcal{Z}}_{j}=\operatorname{Spec} \tilde{R}[[z]]$ for some parameter $z$. We may assume

$$
\beta^{*} z=\chi_{j}(\beta) \cdot z
$$

for all $\beta \in H_{j}$ and some character $\chi_{j}$ with $\chi_{j}^{h_{j}}=\chi$.

The natural map $\mathcal{Y} \rightarrow \mathcal{Z}$ is a $\boldsymbol{\mu}_{p}$-torsor in a neighborhood of $\xi$. Therefore, the pullback to the completion $\hat{\mathcal{Z}}_{j}$ is defined by a Kummer equation

$$
y^{p}=u=\sum_{\mu \geq 0} c_{\mu} z^{\mu}
$$

with $c_{\mu} \in \tilde{R}$ and $c_{0} \in \tilde{R}^{\times}$. The unit $u \in \tilde{R}[[z]]^{\times}$is unique up to a $p$ th power. As we have already remarked above, the $\boldsymbol{\mu}_{p}$-torsor $\hat{\mathcal{Y}}_{j} \rightarrow \hat{\mathcal{Z}}_{j}$ is the pullback of a $\boldsymbol{\mu}_{p}$-torsor defined over $\tilde{R}_{j}$. In other words, we can choose the parameter $z$ and the Kummer equation (16) in such a way that all coefficients $c_{\mu}$ lie in $\tilde{R}_{j} \subset \tilde{R}$. Hence we may and will assume that $c_{\mu} \in \tilde{R}_{j}$.

Lemma 3.5. Let $a_{j}:=h_{j}-m_{j}$. We may choose the parameter $z$, the Kummer equation (16) and a parameter $t_{j}$ for $\tilde{R}_{j}$ such that

$$
u \equiv 1 \bmod \left(p^{2}, z^{a_{j}}\right)
$$

and

$$
u \equiv 1+t_{j} z^{a_{j}}+z^{h_{j}} \bmod \left(p, t_{j} z^{a_{j}+1}, z^{h_{j}+1}\right) .
$$

Proof. Let $\beta$ be a generator of $H_{j} \cong \mathbb{Z} / m_{j}$ and set $\zeta:=\chi_{j}(\beta)$. Note that $\zeta^{m_{j}}=1$ and $\zeta^{a_{j}}=\chi(\beta)$. Choose an integer $1<s<p$ such that $s \equiv \zeta^{a_{j}} \bmod p$. The subgroup scheme $\mathcal{G}_{j}:=\boldsymbol{\mu}_{p} \cdot H_{j} \subset \mathcal{G}$ acts on $\hat{\mathcal{Y}}_{j}$, extending the action of $H_{j}$ on $\hat{\mathcal{Z}}_{j}$ given by (15). Therefore, there exists a unit $w \in \tilde{R}_{j}[[z]]^{\times}$such that $\beta^{*} y=w y^{s}$. Taking $p$ th powers on both sides, we obtain $\beta^{*} u=w^{p} u^{s}$.

If we replace $y$ by $y^{\prime}:=v y$, where $v \in \tilde{R}_{j}[[z]]^{\times}$, then $u$ gets replaced by $u^{\prime}:=v^{p} u$ and $w$ gets replaced by $w^{\prime}:=w\left(\beta^{*} v\right) / v^{s}$. We claim that, by a judicious choice of $v$, we can achieve that $w \equiv 1 \bmod \left(p, z^{a_{j}}\right)$. To prove this claim, we may use induction on the $z$-degree of the error term. We start in degree zero: there we have $\left(\beta^{*} v\right) / v^{s} \equiv$ $v^{1-s}(\bmod z)$. So if we choose $v$ such that $v \equiv w^{1 /(s-1)} \bmod (p, z)$, then we get $w^{\prime} \equiv 1 \bmod (p, z)$. Hence we may assume $w \equiv 1+w_{\mu} z^{\mu} \bmod \left(p, z^{\mu+1}\right)$ for some $c_{\mu} \in \tilde{R}_{j}$, with $0<\mu<a_{j}$. Note that $\zeta^{\mu} \not \equiv s(\bmod p)$. Setting $v:=1-w_{\mu} z /\left(\zeta^{\mu}-s\right)$, we obtain $w^{\prime} \equiv 1 \bmod \left(p, z^{\mu+1}\right)$. The claim now follows by induction. 
By what we have just shown, we may assume $w \equiv 1 \bmod \left(p, z^{a_{j}}\right)$. In particular, we get

$$
\beta^{*} u=w^{p} u^{s} \equiv u^{s} \bmod \left(p^{2}, z^{a_{j}}\right)
$$

Comparing coefficients in $z$ of degree less than $a_{j}$ gives $u \equiv 1 \bmod \left(p^{2}, z^{a_{j}}\right)$, which is the first congruence stated in the lemma.

For $c \in \tilde{R}_{j}$, let $\bar{c}$ denote its image in $k=\tilde{R}_{j} /\left(p, t_{j}\right)$. By construction, the differential form $\mathrm{d} \bar{u}$ has a zero of order $h_{j}-1$ at $z=0$. Since $a_{j} \not \equiv 0(\bmod p)$, this shows that $\bar{c}_{a_{j}}=0$ and $\bar{c}_{h_{j}} \neq 0$. Replacing $z$ by $z^{\prime}:=c_{h_{j}}^{1 / h_{j}} z$ (possible since $h_{j} \not \equiv 0$ $(\bmod p)$ ), we may assume that $c_{h_{j}}=1$. Also, with the same arguments as above, one shows that, up to replacing $y$ by $y^{\prime}=v y$ for some $v \equiv 1 \bmod \left(p, z^{a_{j}}\right)$, we may assume that $w \equiv 1 \bmod \left(p, t_{j}, z^{h_{j}}\right)$. Then the congruence

$$
\beta^{*} u=w^{p} u^{s} \equiv u^{s} \bmod \left(p, t_{j}, z^{h_{j}}\right)
$$

holds. Comparing coefficients in $z$, we find that

$$
u \equiv 1+c t_{j} z^{a_{j}}+z^{h_{j}} \quad \bmod \left(p, t_{j} z^{a_{j}+1}, z^{h_{j}+1}\right)
$$

for some element $c \in \tilde{R}_{j}$. It remains to prove that $\bar{c} \neq 0$, because then we can replace $t_{j}$ by $c^{-1} t_{j}$ and obtain the second congruence stated in the lemma. Let $k[\epsilon]$ be the ring of dual numbers over $k$, and $\kappa: R_{j} \rightarrow k[\epsilon]$ the unique $W(k)$-algebra homomorphism with $\kappa\left(t_{j}\right)=\epsilon$. Write

$$
\kappa(u)=\bar{u}+\epsilon \cdot \bar{v}=\left(1+z^{h_{j}}+\sum_{\mu \geq h_{j}} \bar{c}_{\mu} z^{\mu}\right)+\epsilon \cdot\left(\sum_{\mu \geq a_{j}} \bar{d}_{\mu} z^{\mu}\right)
$$

with $\bar{c}_{\mu}, \bar{d}_{\mu} \in k$. We have to show that $\bar{d}_{a_{j}} \neq 0$.

By the proof of Wew02, Lemma 5.12, there exists an $H_{j}$-invariant derivation of $k[[z]]\left[z^{-1}\right]$

$$
\theta_{j}=\left(\bar{e}_{0}+\bar{e}_{1} x+\ldots\right) \frac{\mathrm{d}}{\mathrm{d} x}=\frac{z^{1-m_{j}}}{m_{j}}\left(\bar{e}_{0}+\bar{e}_{1} z^{m_{j}}+\ldots\right) \frac{\mathrm{d}}{\mathrm{d} z}
$$

(which is the same thing as a derivation of $k[[x]]$, with $x:=z^{m_{j}}$ ) such that the following hold:

(a) $\bar{v}=\theta_{j}(\bar{u})$, and

(b) we have $\bar{e}_{0}=0$ if and only if the pullback of $\hat{\mathcal{Y}}_{j}$ via the homomorphism $\kappa: \tilde{R}_{j} \rightarrow k[\epsilon]$ is the trivial deformation of $\hat{Y}_{j}$.

In fact, the class of $\theta_{j}$ modulo $k[[x]] x \frac{\mathrm{d}}{\mathrm{d} x}$ represents the isomorphism class of the equivariant deformation $\hat{\mathcal{Y}}_{j} \otimes k[\epsilon]$, via a certain isomorphism

$$
\mathcal{E} x t_{\mathcal{G}}^{1}\left(\Omega_{\bar{Y}}, \mathcal{O}_{\bar{Y}}\right)_{\tau_{j}} \cong \mathcal{T}_{\bar{X}, \tau_{j}} / x \cdot \mathcal{T}_{\bar{X}, \tau_{j}}
$$

see Wew02, $§ 5.3$. But since $\hat{\mathcal{Y}}_{j}$ is a miniversal deformation of $\hat{Y}_{j}$ and $\kappa$ is not constant, $\hat{\mathcal{Y}}_{j} \otimes k[\epsilon]$ must be nontrivial. Therefore,

$$
\bar{d}_{a_{j}}=\frac{h_{j} \bar{e}_{0}}{m_{j}} \neq 0
$$

This proves the lemma. 


\subsection{The stable reduction of the auxiliary cover.}

3.3.1. Let $k$ be an algebraically closed field of characteristic $p>0$ and let $(\bar{Z}, \omega)$ be a normalized special deformation datum over $\bar{X}:=\mathbb{P}_{k}^{1}$ of type $(H, \chi)$. Let $\bar{Y} \rightarrow \bar{X}$ be the $\mathcal{G}$-cover associated to $(\bar{Z}, \omega)$, as defined in $\$ 3.1 .1$. Let $K_{0}$ denote the fraction field of $W(k)$ and let $K / K_{0}$ be a finite extension. We assume that $K$ contains the $p$ th roots of unity. The integral closure $R$ of $W(k)$ inside $K$ is a complete mixed characteristic discrete valuation ring with residue field $k$.

Let $\left(\tau_{j, R}\right)$ be a normalized $B$-tuple over $R$, see $\$ 3.2 .1$ By Corollary 3.3 there exists a unique formal deformation $Y_{R} \rightarrow X_{R}=\mathbb{P}_{R}^{1}$ of the $\mathcal{G}$-cover $\bar{Y} \rightarrow \bar{X}$ with branch locus $\left(\tau_{j, R}\right)$. By Grothendieck's existence theorem, this formal deformation is effective, i.e. we may regard $Y_{R} \rightarrow X_{R}$ as a finite morphism of schemes over $R$. Write $Y_{K} \rightarrow X_{K}=\mathbb{P}_{K}^{1}$ for the generic fiber of $Y_{R} \rightarrow X_{R}$ and $x_{j}:=\tau_{j, K}$ for the $K$-rational point on $X_{K}$ corresponding to the section $\tau_{j, R}$, for $j \in B$. Let

$$
G:=\mathcal{G}(K) \cong \mathbb{Z} / p \rtimes_{\chi} H
$$

be the group of $K$-rational points of $\mathcal{G}$. The group $G$ acts on $Y_{K}$, and it is easy to see that $Y_{K} \rightarrow X_{K}$ is a $G$-Galois cover with branch locus $\left(x_{j}\right)$. The ramification index of this cover at the branch point $x_{j}$ is equal to $m_{j}$ for $j \in B_{\text {tame }}=B_{\text {prim }} \cup B_{\text {new }}$, and equal to $p$ for $j \in B_{\text {wild. }}$. Write $Y \rightarrow X:=\mathbb{P}_{\bar{K}}^{1}$ for the pullback of $Y_{K} \rightarrow X_{K}$ to $\bar{K}$.

Definition 3.6. The $G$-cover $f: Y \rightarrow X$ is called the lift of the special deformation datum $(\bar{Z}, \omega)$ with branch locus $\left(x_{j}\right)$.

This definition is justified by the next proposition.

Proposition 3.7. Let $G^{\prime}$ be a finite group, isomorphic to the semi-direct product $\mathbb{Z} / p \rtimes H$ determined by the character $\chi$. Let $W \rightarrow X$ be a $G^{\prime}$-Galois cover. Then $W \rightarrow X$ and $Y \rightarrow X$ are isomorphic as mere covers if and only if the following conditions hold.

(1) The branch locus of $W \rightarrow X$ is equal to $\left\{x_{j} \mid j \in B\right\}$.

(2) The cover $W \rightarrow X$ has bad reduction and the deformation datum occurring over the original component of the stable reduction is isomorphic to $(\bar{Z}, \omega)$.

Proof. Let $W \rightarrow X$ be a $G^{\prime}$-Galois cover satisfying conditions (1) and (2) of the proposition. After replacing $K$ by a larger field, if necessary, we may suppose that $W \rightarrow X$ has a model $W_{K} \rightarrow X_{K}$ over $K$ which extends to a stable model $W_{R}^{\text {st }} \rightarrow X_{R}^{\text {st. }}$. Let $W_{R}$ denote the normalization of $X_{R}=\mathbb{P}_{R}^{1}$ in the function field of $W_{K}$. We obtain a canonical map $W_{R}^{\text {st }} \rightarrow W_{R}$ which is an isomorphism except in the singular points of $\bar{W}$. By condition (2), the action of $G^{\prime}$ on $\bar{W}$ has a kernel $I^{\prime} \triangleleft G^{\prime}$, cyclic of order $p$. By the assumption on $G^{\prime}$ and $W \rightarrow X$, we have $G^{\prime} / I^{\prime} \cong H$, and there exists a finite map $\bar{W} \rightarrow \bar{Z}$, purely inseparable of degree $p$ and $H$-equivariant. Clearly, the isomorphism $G^{\prime} / I^{\prime} \cong H$ extends to an isomorphism $G^{\prime} \cong G$.

Let $\mathcal{G}^{\prime}$ be the schematic closure of $G^{\prime}=\operatorname{Aut}\left(W_{K} / X_{K}\right)$ inside the $R$-group scheme $\operatorname{Aut}\left(W_{R} / X_{R}\right)$. By Ray74, $\mathcal{G}^{\prime}$ is a finite flat group scheme over $R$ with generic fiber $G^{\prime}$. We claim that the isomorphism $G^{\prime} \cong G$ chosen above extends to an isomorphism $\mathcal{G}^{\prime} \cong \mathcal{G} \otimes_{W(k)} R$ of $R$-group schemes. Indeed, it suffices to show that the closure of $I^{\prime}$ inside $\mathcal{G}^{\prime}$ is isomorphic to $\boldsymbol{\mu}_{p}$ (because then $\mathcal{G}^{\prime}$ is the minimal prolongation of $G^{\prime}$ over $R$, see [Ray74]). Therefore, the claim follows from condition (2). 
It is no loss of generality to identify $\mathcal{G}^{\prime}$ with $\mathcal{G} \otimes_{W(k)} R$. So $\mathcal{G}$ acts on $\bar{W}$ such that $\bar{Z}=\bar{W} / \boldsymbol{\mu}_{p}$ and $\bar{X}=\bar{W} / \mathcal{G}$. Moreover, the restriction of the map $\bar{W} \rightarrow \bar{Z}$ to a sufficiently small open subset is a $\boldsymbol{\mu}_{p}$-cover corresponding to the differential $\omega$. We conclude that there exists a $\mathcal{G}$-equivariant isomorphism $\bar{W} \stackrel{\sim}{\rightarrow} \bar{Y}$, see the proof of Proposition 3.1. Via this isomorphism, we may regard $W_{R}$ as an equivariant deformation of $\bar{Y}$. By condition (1), the two $\mathcal{G}$-covers $Y_{R} \rightarrow X_{R}$ and $W_{R} \rightarrow X_{R}$ have the same branch locus $\left(\tau_{j, R}\right)$. Hence the proposition follows from Corollary 3.3 .

3.3.2. Let $\left(\tau_{j, R}\right)$ be a normalized $B$-tuple over $R, x_{j}:=\tau_{j, K} \in X:=\mathbb{P}_{\bar{K}}^{1}$, and let $Y \rightarrow X$ be the lift of $(\bar{Z}, \omega)$ with branch locus $\left(x_{j}\right)$. After replacing $K$ by a finite extension, we may assume that the natural $K$-model $Y_{K} \rightarrow X_{K}$ extends to the stable model $Y_{R}^{\text {st }} \rightarrow X_{R}^{\text {st }}$. We denote by $\bar{Y}^{\text {st }} \rightarrow \bar{X}^{\text {st }}$ the stable reduction and by $\bar{x}_{j} \in \bar{X}^{\text {st }}$ the specialization of the branch point $x_{j}$. The stable model is obtained as a blow-up of the natural $R$-model $Y_{R} \rightarrow X_{R}$, i.e. we have a commutative diagram

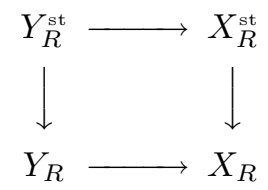

in which the vertical arrows are modifications which are isomorphisms away from the critical points of $\bar{X}$ (resp. the singular points of $\bar{Y}$ ). In particular, the map $\bar{X}^{\text {st }} \rightarrow \bar{X}=\mathbb{P}^{1}$ induces an isomorphism between $\bar{X}$ and the original component $\bar{X}_{0}$ of $\bar{X}^{\text {st }}$, and contracts all other components to one of the critical points $\tau_{j}$, $j \in B_{\text {prim }} \cup B_{\text {new }}$. By construction of $Y_{R}$, the deformation datum over $\bar{X}_{0} \cong \bar{X}$ associated to $\bar{Y}^{\text {st }} \rightarrow \bar{X}^{\text {st }}$ is isomorphic to $(\bar{Z}, \omega)$.

Here is the main result of this section.

Theorem 3.8. The following two conditions are equivalent:

(1) The curve $\bar{X}^{\text {st }}$ consists only of the original component $\bar{X}_{0}$ and, for each $j \in B_{\text {tame }}$, a tail $\bar{X}_{j}$ which contains $\bar{x}_{j}$ and intersects $\bar{X}_{0} \cong \bar{X}$ in $\tau_{j}$.

(2) For all $j \in B_{\text {new }}$ we have

$$
\operatorname{val}\left(\tau_{j, R}-\left[\tau_{j}\right]\right) \geq \frac{p m_{j}}{(p-1) h_{j}} \cdot \operatorname{val}(p) .
$$

(Recall that $\left[\tau_{j}\right] \in W(k) \subset R$ denotes the Teichmüller lift of $\tau_{j}$. )

Remark 3.9. Under the additional assumption $B_{\text {wild }}=\emptyset$, the implication ' $(2) \Rightarrow$ (1)' is already proved in Wew03.

Proof. The element $\tau_{j, R}-\left[\tau_{j}\right] \in R$ is the image of the parameter $T_{j}$ (defined in \$3.2.2) under the classifying morphism $\tilde{R} \rightarrow R$ of the equivariant deformation $Y_{R}$. By abuse of notation, we write $T_{j}:=\tau_{j, R}-\left[\tau_{j}\right]$ as an element of $R$.

Let us first prove the implication ' $(2) \Rightarrow(1)$ ', i.e. we assume that (2) holds. To find the modification $Y_{R}^{\text {st }} \rightarrow Y_{R}$ which leads to the stably marked model of $Y_{R}$, it suffices to consider the completion of $Y_{R}$ at a singular point of $\bar{Y}$. Let $\eta_{j} \in \bar{Y}$ be one of the singular points, lying above the critical point $\tau_{j} \in \bar{X}$ for $j \in B_{\text {tame }}$. Let $\xi_{j} \in \bar{Z}$ be the image of $\eta_{j}$. Let $\hat{Y}_{R, j}$ denote the completion of $Y_{R}$ at $\eta_{j}$ and $\hat{Z}_{R, j}$ the completion of $Z_{R}$ at $\xi_{j}$. Condition (1) of the theorem means that the germ $\hat{Y}_{R, j}$ has potentially good reduction. We can write $\hat{Z}_{R, j}=\operatorname{Spec} R[[z]]$. The $\boldsymbol{\mu}_{p}$-torsor $\hat{Y}_{R, j} \rightarrow \hat{Z}_{R, j}$ is given by a Kummer equation $y^{p}=u$, with $u \in R[[z]]^{\times}$. 
Let us first treat the case $j \in B_{\text {new }}$. By Lemma 3.5 and Proposition 3.4 (3) we may assume that

$$
y^{p}=u \equiv 1+w_{j} T_{j} z^{a_{j}}+z^{h_{j}} \bmod \left(p, T_{j} z^{a_{j}+1}, z^{h_{j}+1}\right),
$$

with $w_{j} \in R^{\times}$, and

$$
u \equiv 1 \bmod \left(p^{2}, z^{a_{j}}\right) .
$$

We also may assume that $R$ contains an element $\lambda$ such that $\lambda^{h_{j}(p-1)}=-p$. Let $y=1+\lambda^{h_{j}} y^{\prime}$ and $z=\lambda^{p} z^{\prime}$. The new coordinates $y^{\prime}$ and $z^{\prime}$ give rise to a diagram

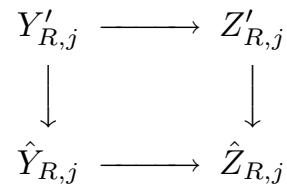

in which the vertical arrows are blow-ups centered in the closed point of the germs $\hat{Y}_{R, j}$ and $\hat{Z}_{R, j}$, respectively. Let $\bar{Y}_{j}^{\prime}$ (resp. $\bar{Z}_{j}^{\prime}$ ) be the exceptional divisor of these blowups. The curve $\bar{Z}_{j}^{\prime}$ is a projective line with parameter $z^{\prime}$. To obtain the equation for the cover $\bar{Y}_{j}^{\prime} \rightarrow \bar{Z}_{j}^{\prime}$, rewrite (18) in terms of $z^{\prime}$ and $y^{\prime}$, subtract 1 , divide by $\lambda^{p h_{j}}$ and reduce modulo the maximal ideal of $R$. Using the assumption $(p-1) h_{j} \operatorname{val}\left(T_{j}\right) \geq p m_{j} \operatorname{val}(p)$ and the congruences (18) and (19), one obtains an Artin-Schreier equation

$$
y^{\prime p}-y^{\prime}=\bar{w}_{j} z^{\prime a_{j}}+z^{h_{j}}
$$

with conductor $h_{j}$ at $z^{\prime}=\infty$. It follows that $\bar{Y}_{j}^{\prime}$ is smooth and that $Y_{R, j}^{\prime}$ is a semistable model of the germ $\hat{Y}_{R, j}$. In other words, the germ $\hat{Y}_{R, j}$ has potentially good reduction. See, e.g., Ray99], Lemme 4.3.3, or [Leh01], §3. We conclude that condition (1) holds for the given index $j \in B_{\text {new }}$.

Condition (1) holds for $j \in B_{\text {prim }}$ as well, without any assumption. Indeed, if $j \in B_{\text {prim }}$, then $0<h_{j}<m_{j}$. An easy version of the proof of Lemma 3.5 shows that (for the right choice of parameter $z$ ) the $\boldsymbol{\mu}_{p}$-torsor $Y_{R, j} \rightarrow Z_{R, j}$ is given by a Kummer equation of the form

$$
y^{p}=u \equiv 1+z^{h_{j}} \bmod \left(p^{2}, z^{h_{j}+1}\right) .
$$

After a change of coordinate $y=1+\lambda^{h_{j}} y^{\prime}$ and $z^{\prime}=\lambda^{p} z^{\prime}$ we again find an ArtinSchreier equation with conductor $h_{j}$, which shows that the germ $\hat{Y}_{R, j}$ has potentially good reduction. This finishes the proof of the implication ' $(2) \Rightarrow(1)$ '.

It remains to prove the implication ' $(1) \Rightarrow(2)$ '. Suppose that condition $(2)$ is violated for some $j \in B_{\text {new }}$. We may also assume that $R$ contains an element $\lambda$ with $\lambda^{p m_{j}}=T_{j}$. After substituting $y=1+\lambda^{h_{j}} y^{\prime}$ and $z=\lambda^{p} z^{\prime}$ into (18) and reducing modulo the maximal ideal of $R$, we find that the cover $\bar{Y}_{j}^{\prime} \rightarrow \bar{Z}_{j}^{\prime}$ has equation

$$
y^{\prime p}=\bar{w}_{j} z^{\prime a_{j}}+z^{h_{j}} .
$$

It follows that the cover $\bar{Y}_{j}^{\prime} \rightarrow \bar{Z}_{j}^{\prime}$ is an $\boldsymbol{\alpha}_{p}$-torsor (away from the point $z^{\prime}=\infty$ ) corresponding to the exact differential $\omega^{\prime}=\mathrm{d}\left(\bar{w}_{j} z^{\prime a_{j}}+z^{\prime h_{j}}\right)$. The differential $\omega^{\prime}$ has a zero of order $a_{j}-1$ at $z^{\prime}=0$ and $m_{j}$ further simple zeros. Above each point where $\omega^{\prime}$ has a zero, $\bar{Y}_{j}^{\prime}$ has a singularity. We conclude that the germ $\hat{Y}_{R, j}$ does not have potentially good reduction. In other words, condition (1) of Theorem 3.8 does not hold. This finishes the proof of Theorem 3.8 . 
The proof shows that, if both the conditions of the theorem hold, then the closed rigid disk

$$
D_{j}:=\left\{x \mid \operatorname{val}\left(x-\left[\tau_{j}\right]\right) \geq \frac{p m_{j}}{(p-1) h_{j}} \cdot \operatorname{val}(p)\right\} \subset X_{K}^{\text {rig }}
$$

is equal to the set of points on $X_{K}$ which specialize to the tail $\bar{X}_{j} \subset X_{R}^{\text {st. }}$. We remark at this point that, since $\frac{p m_{j}}{(p-1) h_{j}}<1$ for $j \in B_{\text {new }}$, the family of disks $\left(D_{j}\right)_{j \in B}$ is independent of the choice of $\left(\tau_{j, R}\right)$, as long as condition (2) of Theorem 3.8 holds.

3.3.3. Let us now assume that the branch points $x_{j}$ of the lift $f: Y \rightarrow X$ of $(\bar{Z}, \omega)$ are all $K_{0}$-rational. This is equivalent to the condition that $\tau_{j, R} \in W(k)$. Then condition (2) of Theorem 3.8 is automatically satisfied. Moreover, the cover $f: Y \rightarrow X$ has a natural model $f_{K_{0}}: Y_{K_{0}} \rightarrow X_{K_{0}}=\mathbb{P}_{K_{0}}^{1}$ over $K_{0}$ (which is not Galois, since $K_{0}$ does not contain the $p$ th roots of unity). Let

$$
\bar{\kappa}^{\mathrm{ab}}: \operatorname{Gal}\left(\bar{K} / K_{0}\right) \longrightarrow \operatorname{Aut}_{k}\left(\bar{Y}^{\mathrm{st}}\right)
$$

be the absolute monodromy action associated to the $K_{0}$-model $f_{K_{0}}$ of $f$, see 1.1 .3 The goal of this subsection is to describe this action in detail.

First some notation. Let $\bar{Y}_{0}$ be the unique irreducible component of $\bar{Y}^{\text {st }}$ which lies above the original component $\bar{X}_{0} \subset \bar{X}^{\text {st }}$. We may identify $\bar{Y}_{0}$ with the normalization of $\bar{Y}$ or, what amounts to the same thing, the smooth projective model of the function field $k(\bar{Z})^{1 / p}$. Let us choose, for all $j \in B_{\text {tame }}$, a point $\eta_{j} \in \bar{Y}_{0}$ above $\tau_{j} \in \bar{X}$. We denote by $\xi_{j} \in \bar{Z}$ the image of $\eta_{j}$, by $H_{j} \subset H$ the stabilizer of $\xi_{j}$ and by $I \subset G$ the cyclic subgroup of order $p$. Set $G_{j}:=I \cdot H_{j} \subset G$. Let $\bar{Y}_{j} \subset \bar{Y}^{\text {st }}$ (resp. $\bar{X}_{j} \subset \bar{X}^{\text {st }}$ ) be the component which intersects $\bar{Y}_{0}$ in $\eta_{j}$ (resp. intersects $\bar{X}$ in $\tau_{j}$ ). By Theorem 3.8 $\bar{X}^{\text {st }}=\bar{X} \cup \bigcup_{j} \bar{X}_{j}$. Furthermore, the proof of the theorem shows that $\left(\bar{Y}_{j} \rightarrow \bar{X}_{j}, \eta_{j}, H_{j}\right)$ is a pointed $G_{j}$-tail cover with ramification type $\left(m_{j}, h_{j}\right)$ which is totally ramified above $\tau_{j} \in \bar{X}_{j}$, see $\$ 2.2 .2$.

Let us denote by $\operatorname{Aut}_{k}^{0}\left(\bar{Y}^{\text {st }}\right)$ the group of $k$-linear automorphisms of $\bar{Y}^{\text {st }}$ which normalize the action of $G$, commute with the action of $H \subset G$ and induce the identity on $\bar{Y}_{0}$. Similarly, let $\operatorname{Aut}_{k}^{0}\left(\bar{Y}_{j}\right)$ denote the group of (outer) automorphisms of the pointed $G_{j}$-tail cover $\bar{f}_{j}$. Recall that these are $k$-linear automorphisms of $\bar{Y}_{j}$ which normalize the action of $G_{j}$, commute with the action of $H_{j} \subset G_{j}$ and fix the point $\eta_{j}$. For each $j$ we have a natural homomorphism

$$
\operatorname{Aut}_{k}^{0}\left(\bar{Y}^{\mathrm{st}}\right) \longrightarrow \operatorname{Aut}_{k}^{0}\left(\bar{Y}_{j}\right) \text {. }
$$

Let $\alpha$ be a generator of $I \triangleleft G$. For $\varphi \in \operatorname{Aut}_{k}^{0}(\bar{Y})$ we have $\varphi \circ \alpha \circ \varphi^{-1}=\alpha^{\rho}$ for some $\rho \in \mathbb{F}_{p}^{\times}$; this defines a character

$$
\rho: \operatorname{Aut}_{k}^{0}(\bar{Y}) \longrightarrow \mathbb{F}_{p}^{\times} .
$$

Similarly, one defines characters $\rho_{j}: \operatorname{Aut}_{k}^{0}\left(\bar{Y}_{j}\right) \rightarrow \mathbb{F}_{p}^{\times}$for all $j \in B_{\text {tame }}$. The composition of $\rho$ with $\bar{\kappa}^{\text {ab }}$ is the cyclotomic character modulo $p$. The kernel of the character $\rho$ is the subgroup $\operatorname{Aut}_{G}^{0}\left(\bar{Y}^{\text {st }}\right) \subset \operatorname{Aut}_{k}^{0}\left(\bar{Y}^{\text {st }}\right)$ of automorphisms which commute with the $G$-action. Compare with 2.2 .4 .

Proposition 3.10. $\quad$ (1) We have a canonical isomorphism

$$
\operatorname{Aut}_{k}^{0}\left(\bar{Y}^{\mathrm{st}}\right) \cong\left\{\left(\rho ; \varphi_{j}\right) \in \mathbb{F}_{p}^{\times} \times \prod_{j} \operatorname{Aut}_{k}^{0}\left(\bar{Y}_{j}\right) \mid \rho=\rho_{j}\left(\varphi_{j}\right) \forall j\right\} .
$$


(2) The group $\operatorname{Aut}_{k}^{0}\left(\bar{Y}_{j}\right)$ is cyclic of order $(p-1) h_{j}$. Therefore, the group $\operatorname{Aut}_{k}^{0}\left(\bar{Y}^{\text {st }}\right)$ has order $(p-1) \prod_{j} h_{j}$.

(3) The restriction of $\bar{\kappa}^{\text {ab }}$ to the component $\bar{Y}_{j}$ is a surjective homomorphism

$$
\bar{\kappa}_{j}^{\mathrm{ab}}: \operatorname{Gal}\left(\bar{K} / K_{0}\right) \longrightarrow \operatorname{Aut}_{k}^{0}\left(\bar{Y}_{j}\right) .
$$

(4) The image of $\bar{\kappa}^{\mathrm{ab}}$ is a cyclic subgroup of $\operatorname{Aut}_{k}^{0}\left(\bar{Y}^{\mathrm{st}}\right)$, of order

$$
N:=(p-1) \cdot \operatorname{lcm}_{j}\left(h_{j}\right) .
$$

Proof. Part (1) is left to the reader. We also leave it to the reader to check that the image of the monodromy action $\bar{\kappa}^{\text {ab }}$ is contained in $\operatorname{Aut}_{k}^{0}\left(\bar{Y}^{\mathrm{st}}\right)$.

The explicit description of the change of coordinates $y=1+\lambda^{h_{j}} y^{\prime}, z=\lambda^{p} z^{\prime}$ in the proof of Theorem 3.8 shows that the restriction of $\bar{\kappa}^{\text {ab }}$ to $\bar{Y}_{j}$ is cyclic of order $(p-1) h_{j}$ (it corresponds to the cyclic Galois extension $K_{0}(\lambda) / K_{0}$, where $\left.\lambda^{(p-1) h_{j}}=-p\right)$. In particular, the group $\operatorname{Aut}_{k}^{0}\left(\bar{Y}_{j}\right)$ has a cyclic subgroup of order $(p-1) h_{j}$. Therefore, in order to prove the proposition it suffices to show that the group $\operatorname{Aut}_{k}^{0}\left(\bar{Y}_{j}\right)$ has exact order $(p-1) h_{j}$.

First, we claim that $\operatorname{Aut}_{k}^{0}\left(\bar{Y}_{j}\right)$ is cyclic. Indeed, the natural action of $\operatorname{Aut}_{k}^{0}\left(\bar{Y}_{j}\right)$ on $\bar{Y}_{j}$ descends to a faithful action on the quotient $\bar{X}_{j}=\bar{Y}_{j} / G_{j}$ which fixes the two points $\tau_{j}$ and $\bar{x}_{j}$. But $\bar{X}_{j}$ has genus 0 , so the claim follows. Now assume that $\operatorname{Aut}_{k}^{0}\left(\bar{Y}_{j}\right)$ has order $(p-1) h_{j} n$. Let $\varphi \in \operatorname{Aut}_{k}^{0}\left(\bar{Y}_{j}\right)$ be an element of order $h_{j} n$. Since $\varphi$ lies in the kernel of the character $\rho_{j}$, it commutes with the action of $G_{j}$ on $\bar{Y}_{j}$. Let $\bar{Y}_{j}^{\prime}:=\bar{Y}_{j} /\langle\varphi\rangle \rightarrow \bar{X}_{j}^{\prime}:=\bar{X}_{j} /\langle\varphi\rangle$ be the induced $G_{j}$-cover. Recall that the action of $I \cong \mathbb{Z} / p$ on $\bar{Y}_{j}$ has conductor $h_{j}$ at the point $\eta_{j}$. Let $\eta_{j}^{\prime}$ denote the image of $\eta_{j}$ on $\bar{Y}_{j}^{\prime}$. A classical calculation (see, e.g., Ray99, Lemme 1.1.2) shows that the action of $I$ on $\bar{Y}_{j}^{\prime}$ has conductor $1 / n$ at $\eta_{j}^{\prime}$. We conclude that $n=1$. This finishes the proof of the proposition.

The proposition implies that the $G$-cover $f: Y \rightarrow X$ has stable reduction over the unique tame extension $K^{\text {st }} / K_{0}$ of degree $N$.

\section{Construction of three POINT COVERS By Lifting}

Let $\bar{f}: \bar{Y} \rightarrow \bar{X}$ be a special $G$-map, see Definition [2.15, A lift of $\bar{f}$ is a three point $G$-cover with stable reduction $\bar{f}$. Let $L(\bar{f})$ denote the set of isomorphism classes of all lifts of $\bar{f}$. The Galois group $\operatorname{Gal}\left(\bar{K} / K_{0}\right)$ acts on $L(\bar{f})$ in a natural way. In this section we determine the structure of $L(\bar{f})$ as a $\operatorname{Gal}\left(\bar{K} / K_{0}\right)$-set. In particular, we prove that $L(\bar{f})$ is nonempty and that the action of $\operatorname{Gal}\left(\bar{K} / K_{0}\right)$ is tamely ramified.

At the end, we derive various consequences of the above result, concerning the field of moduli of three point covers, and we discuss a particular class of examples, called genus zero dessins of prime degree.

\subsection{The set of lifts of $\bar{f}$.}

4.1.1. Let $k$ be an algebraic closure of the finite field $\mathbb{F}_{p}, W(k)$ the ring of Witt vectors over $k$ and $K_{0}$ the fraction field of $W(k)$. We fix an algebraic closure $\bar{K}$ of $K_{0}$. In this section, $K / K_{0}$ always denotes a finite field extension contained in $\bar{K}$ and $R$ denotes the ring of integers of $K$. Let $\left(\bar{Z}_{0}, \omega_{0} ; \bar{Y}_{j}\right)$ be a special $G$-deformation datum and let $\bar{f}: \bar{Y} \rightarrow \bar{X}$ be the associated special $G$-map, see $\$ 2.2$. 
Definition 4.1. A lift of $\bar{f}$ over $R$ is a stable $G$-map $f_{R}: Y_{R} \rightarrow X_{R}$ over $R$ (see Definition [2.14), together with an isomorphism $\bar{f} \cong f_{R} \otimes_{R} k$ of stable $G$-maps and an isomorphism $X_{R} \otimes_{R} K \stackrel{\sim}{\rightarrow}\left(\mathbb{P}_{K}^{1},\{0,1, \infty\}\right)$ of marked curves. Given a lift $f_{R}: Y_{R} \rightarrow X_{R}$, we will usually identify $\bar{f}$ with the special fiber of $f_{R}$ and $\mathbb{P}_{K}^{1}$ with the general fiber of $X_{R}$. Two lifts $f_{R}: Y_{R} \rightarrow X_{R}$ and $f_{R}^{\prime}: Y_{R}^{\prime} \rightarrow X_{R}^{\prime}$ over $R$ are said to be isomorphic if there exists an isomorphism $f_{R} \stackrel{\sim}{\rightarrow} f_{R}^{\prime}$ of stable $G$-maps which induces the identity on the special fiber and on $X_{K}=X_{K}^{\prime}=\mathbb{P}_{K}^{1}$.

If $f_{R}: Y_{R} \rightarrow X_{R}$ is a lift of $\bar{f}$, then the general fiber $f_{K}: Y_{K} \rightarrow X_{K}=\mathbb{P}_{K}^{1}$ of $f_{R}$ is a three point $G$-cover and $f_{R}$ is its stable model. In particular, $f_{K}$ has mild reduction and $\bar{f}$ is the stable reduction of $f_{K}$. If $f_{R}$ and $f_{R}^{\prime}$ are isomorphic lifts of $\bar{f}$, then the isomorphism $f_{R} \stackrel{\sim}{\rightarrow} f_{R}^{\prime}$ is unique. (This is a general property of deformations of stably marked curves, see [Knu83].)

4.1.2. Let $L(\bar{f}, K)$ denote the set of isomorphism classes of lifts of $\bar{f}$ over $R$, the ring of integers of $K$. If $K^{\prime} / K$ is a finite extension then we have a natural inclusion $L(\bar{f}, K) \hookrightarrow L\left(\bar{f}, K^{\prime}\right)$. Let

$$
L(\bar{f}):=\bigcup_{K} L(\bar{f}, K)
$$

be the union, taken over all finite extensions $K / K_{0}$ contained in $\bar{K}$. It is clear that $L(\bar{f})$ is a finite set. A priori, $L(\bar{f})$ may be empty; we will see later that it is not.

There is a natural continuous action of $\operatorname{Gal}\left(\bar{K} / K_{0}\right)$ on $L(\bar{f})$ from the left, defined as follows. Let $f_{R}: Y_{R} \rightarrow X_{R}$ be a lift of $\bar{f}$ over $R$, the ring of integers of $K$, and let $\sigma \in \operatorname{Gal}\left(\bar{K} / K_{0}\right)$. We may assume that $K / K_{0}$ is Galois. Let ${ }^{\sigma} f_{R}:{ }^{\sigma} Y_{R} \rightarrow{ }^{\sigma} X_{R}$ be the pullback of $f_{R}$ by the automorphism $\tilde{\sigma}$ : Spec $R \stackrel{\sim}{\rightarrow}$ Spec $R$ induced by $\sigma$. Since $\sigma$ induces the identity on the residue field $k$, we have a canonical isomorphism $f_{R} \otimes_{R} k \stackrel{\sim}{\rightarrow}{ }^{\sigma} f_{R} \otimes_{R} k$. We may therefore consider ${ }^{\sigma} f_{R}$ as a deformation of $\bar{f}$. This defines the action of $\operatorname{Gal}\left(\bar{K} / K_{0}\right)$ on $L(\bar{f})$.

Recall that $\operatorname{Aut}_{G}^{0}(\bar{f})$ was defined in $\$ 2.2 .4$ as the group of automorphisms of $\bar{f}$ which induce the identity on the original component. There is a natural right action of $\operatorname{Aut}_{G}^{0}(\bar{f})$ on $L(\bar{f})$, defined as follows. If $\gamma \in \operatorname{Aut}_{G}^{0}(\bar{f})$ and $f_{R}: Y_{R} \rightarrow X_{R}$ is a lift of $\bar{f}$, then we define $f_{R}^{\gamma}$ as the stable $G$-map $f_{R}: Y_{R} \rightarrow X_{R}$ together with the isomorphism $\bar{Y} \stackrel{\sim}{\rightarrow} Y_{R} \otimes_{R} k$ which is the composition of $\gamma: \bar{Y} \stackrel{\sim}{\rightarrow} \bar{Y}$ with the canonical isomorphism $\bar{Y} \stackrel{\sim}{\rightarrow} Y_{R} \otimes_{R} k$. This defines an action of $\operatorname{Aut}_{G}^{0}(\bar{f})$ on $L(\bar{f})$ from the right which commutes with the action of $\operatorname{Gal}\left(\bar{K} / K_{0}\right)$. We define

$$
\tilde{L}(\bar{f}):=L(\bar{f}) / \operatorname{Aut}_{G}^{0}(\bar{f}) .
$$

It is clear that elements of $\tilde{L}(\bar{f})$ correspond naturally to isomorphism classes of three point $G$-covers $f: Y \rightarrow X$ whose stable reduction is isomorphic to $\bar{f}$. By definition we have

Proposition 4.2. Let $f_{R}: Y_{R} \rightarrow X_{R}$ be a lift of $\bar{f}$ and denote by $f: Y \rightarrow X$ the geometric generic fiber of $f_{R}$.

(1) The stabilizer in $\operatorname{Gal}\left(\bar{K} / K_{0}\right)$ of $f_{R}$, considered as an element of $L(\bar{f})$, is equal to $\Gamma_{f}^{\mathrm{st}}$, as defined in 1.1 .3

(2) The stabilizer in $\operatorname{Gal}\left(\bar{K} / K_{0}\right)$ of $f_{R}$, considered as an element of $\tilde{L}(\bar{f})$, is equal to $\Gamma_{f}^{\mathrm{in}}$, as defined in $\$ 1.1 .3$. 
4.1.3. The auxiliary cover. Let $f_{R}: Y_{R} \rightarrow X_{R}$ be a lift of $\bar{f}$. For $j \in B_{\text {prim }} \cup B_{\text {new }}$, let $D_{j} \subset X_{K}^{\text {rig }}$ denote the closed rigid disk corresponding to the tail $\bar{X}_{j}$. By definition, a $K$-rational point $x \in D_{j}$ specializes to a point $\bar{x} \in \bar{X}_{j}$. In particular, for $j \in B_{\text {prim }}$ the branch point $x_{j} \in\{0,1, \infty\}$ of $f$ lies on $D_{j}$. Note that the model $X_{R}$ of $X_{K}=\mathbb{P}_{K}^{1}$ is uniquely determined by the family of disks $\left(D_{j}\right)_{j}$. A priori, the disks $D_{j}$ (and therefore the model $X_{R}$ ) may depend on the lift $f_{R}$. We are now going to show that they are in fact independent of $f_{R}$. This is an important steps towards our main result.

Let us choose, for all $j \in B_{\text {new }}$, a $K$-rational point $x_{j} \in D_{j}$. We call these points the auxiliary branch points. By Ray99, §3.2, the choice of the auxiliary branch points yields a certain $G_{0}$-cover $f^{\text {aux }}: Y^{\text {aux }} \rightarrow X$, called the auxiliary cover of $f$. By definition, $f^{\text {aux }}$ has branch locus $\left\{x_{j} \mid j \in B\right\}$ and the same type of bad reduction as $f$. To be more precise, let $\hat{X}_{R}$ denote the formal completion of $X_{R}$ along $\bar{X}$ and let $\mathcal{D}_{j} \subset \hat{X}_{R}$, for $j \in B_{\text {prim }} \cup B_{\text {new }}$, denote the open subset corresponding to $\bar{X}_{j}-\left\{\tau_{j}\right\} \subset \bar{X}$, i.e. $\mathcal{D}_{j}$ is the closed formal disk whose generic fiber is $D_{j}$. Let $\mathcal{U}^{\circ} \subset \hat{X}_{R}$ denote the open subset corresponding to $\bar{X}-\bigcup_{j} \bar{X}_{j} \subset \bar{X}$. Finally, let $\mathcal{X}_{j}$ denote the completion of $X_{R}$ at $\tau_{j} \in \bar{X}$ and set $\mathcal{U}:=\mathcal{U}^{\circ} \cup \bigcup_{j} \mathcal{X}_{j}$. Recall that part of our special $G$-deformation datum $\left(\bar{Z}_{0}, \omega_{0} ; \bar{Y}_{j}\right)$ is a subgroup $G_{0}=I_{0} \cdot H_{0}$. The auxiliary cover of the lift $f_{R}: Y_{R} \rightarrow X_{R}$ is a $G_{0}$-cover $f^{\text {aux }}: Y^{\text {aux }} \rightarrow X$, characterized by the following properties, see Ray99, $§ 3.2$.

(a) The cover $f^{\text {aux }}$ has a stable model $f_{R}^{\text {aux }}: Y_{R}^{\text {aux }} \rightarrow X_{R}^{\text {aux }}$ over $R$. Moreover, we have a (unique) isomorphism $X_{R} \cong X_{R}^{\text {aux }}$ extending the identity on the generic fiber.

(b) For $j \notin B_{\text {wild }}$, the restriction of $f_{R}^{\text {aux }}$ to the formal disk $\mathcal{D}_{j}$ is a tame cover of formal schemes, ramified along the closure of $x_{j}$ in $\mathcal{D}_{j}$.

(c) There exists a $G$-equivariant isomorphism

$$
\hat{Y}_{R} \times_{\hat{X}_{R}} \mathcal{U} \cong \operatorname{Ind}_{G_{0}}^{G}\left(\hat{Y}_{R}^{\text {aux }} \times_{\hat{X}_{R}} \mathcal{U}\right)
$$

of formal $\mathcal{U}$-schemes.

It follows that the branch locus of $f^{\text {aux }}: Y^{\text {aux }} \rightarrow X$ is precisely the set of points $\left\{x_{j} \mid j \in B\right\}$.

Proposition 4.3. (1) The cover $f^{\text {aux }}$ is the (unique) lift of $\left(\bar{Z}_{0}, \omega_{0}\right)$ with branch points $\left(x_{j}\right)$, see 3.3.1. In particular, as a mere cover, $f^{\text {aux }}$ does not depend on the lift $f_{R}$ of $\bar{f}$.

(2) The disks $D_{j}$ are independent of the lift $f_{R}: Y_{R} \rightarrow X_{R}$. Moreover, each disk $D_{j}$ contains a $K_{0}$-rational point.

Proof. It follows immediately from property (c) that the deformation datum over the original component associated to the stable reduction of $f^{\text {aux }}$ can be identified with $\left(\bar{Z}_{0}, \omega_{0}\right)$. Hence (1) follows from Proposition 3.7. By property (a), the cover $f^{\text {aux }}$ satisfies condition (1) of Theorem [3.8. Therefore, (2) follows from Theorem 3.8.

We remark that the proof of Proposition 4.3 is the only place where we use the subtle implication ' $(1) \Rightarrow(2)$ ' of Theorem [3.8.

4.2. Patching data. Lifting the special $G$-map $\bar{f}$ to a three point $G$-cover $f$ : $Y \rightarrow X$ can be done in three steps. The first step consists in lifting the tail covers $\bar{f}_{j}: \bar{Y}_{j} \rightarrow \bar{X}_{j}$ to Galois covers $\mathcal{E}_{j} \rightarrow \mathcal{D}_{j}$ of the formal disks $\mathcal{D}_{j}$ corresponding to 
$D_{j} \subset X$. Since the restriction of $\bar{f}_{j}$ to $\bar{X}_{j}-\left\{\tau_{j}\right\}$ is tame, such a lift exists and is unique. The second step consists in lifting the (inseparable) cover $\bar{Y}_{0} \rightarrow \bar{X}_{0}$ to a Galois cover $\mathcal{V} \rightarrow \mathcal{U}$, where $\mathcal{U}$ is the 'formal neighborhood' of $\hat{X}_{R}-\bigcup_{j} \mathcal{D}_{j}$ defined in 4.1.3 The existence and uniqueness of such a lift follows from the results of 3.1. In the third step we patch the covers $\mathcal{E}_{j} \rightarrow \mathcal{D}_{j}$ and $\mathcal{V} \rightarrow \mathcal{U}$ together along the boundaries $\mathcal{B}_{j}$ of the disks $\mathcal{D}_{j}$ and obtain the desired $G$-cover $f: Y \rightarrow X$. Unlike the first two steps, the third step is not unique; it depends on the choice of what we call a patching datum for $\bar{f}$. The construction we have just sketched amounts to the existence of a bijection

$$
L(\bar{f}) \stackrel{\sim}{\longrightarrow} P(\bar{f})
$$

between the set of lifts of $\bar{f}$ and the set $P(\bar{f})$ of all patching data. Essentially by construction, this bijection is $\operatorname{Gal}\left(\bar{K} / K_{0}\right)$-invariant. Moreover, the results of $\$ 3.3 .3$ can be used to determine the complete structure of $P(\bar{f})$ as a $\operatorname{Gal}\left(\bar{K} / K_{0}\right)$ set. Intuitively, the Galois group $\operatorname{Gal}\left(\bar{K} / K_{0}\right)$ acts on $P(\bar{f})$ by 'Dehn twists' along the boundaries $\mathcal{B}_{j}$.

4.2.1. For each index $j \in B$, let us now choose, once and for all, a $K_{0}$-rational point $x_{j} \in X$ such that the closure of $x_{j}$ in $X_{0}=\mathbb{P}_{W(k)}^{1}$ intersects the special fiber $\bar{X}_{0}=\mathbb{P}_{k}^{1}$ in the critical point $\tau_{j}$. As usual, we assume that $x_{j} \in\{0,1, \infty\}$ for $j \in B_{0}$. Let $f^{\dagger}: Y^{\dagger} \rightarrow X=\mathbb{P}_{\bar{K}}^{1}$ be the lift of the special deformation datum $\left(\bar{Z}_{0}, \omega_{0}\right)$ with branch points $\left(x_{j}\right)_{j \in B}$, see 3.3 By definition, $f^{\dagger}$ is a $G^{\dagger}$-cover, where $G^{\dagger}:=\boldsymbol{\mu}_{p}(\bar{K}) \rtimes H_{0}$. If $f_{R}$ is a lift of $\bar{f}$, then Proposition 4.3 shows that $f^{\dagger}$ is isomorphic, as a mere cover, to the auxiliary cover $f^{\text {aux }}$ associated to $f_{R}$. In the following, we will not identify $f^{\dagger}$ with $f^{\text {aux }}$, because we want to emphasize the fact that such an identification is not canonical.

Let $K / K_{0}$ be a finite extension over which $f^{\dagger}$ has stable reduction, and let $f_{R}^{\dagger}: Y_{R}^{\dagger} \rightarrow X_{R}^{\dagger}$ (resp. $\bar{f}^{\dagger}: \bar{Y}^{\dagger} \rightarrow \bar{X}^{\dagger}$ ) denote the stable model (resp. the stable reduction) of $f^{\dagger}$. By Theorem 3.8 the curve $\bar{X}^{\dagger}$ consists of the original component $\bar{X}_{0}=\mathbb{P}_{k}^{1}$ and the tails $\bar{X}_{j}^{\dagger}$, where $j$ runs over the set $B_{\text {prim }} \cup B_{\text {new }}$. Moreover, the deformation datum over the original component associated to $\bar{f}^{\dagger}$ is canonically isomorphic to $\left(\bar{Z}_{0}, \omega_{0}\right)$. We may and will identify the (unique) component $\bar{Y}_{0}^{\dagger}$ of $\bar{Y}^{\dagger}$ over the original component with the distinguished component $\bar{Y}_{0}$ of $\bar{Y}$ (by definition, $\bar{Y}_{0}=\bar{Z}_{0}^{1 / p}$, see $\left.\$ 2.2 .3\right)$. Recall that we have distinguished points $\eta_{j} \in \bar{Y}_{0}$ above $\tau_{j} \in \bar{X}_{0}$; they are part of the $G$-deformation datum $\left(\bar{Z}_{0}, \omega_{0} ; \bar{Y}_{j}\right)$. For $j \notin B_{\text {wild }}$ we let $\bar{Y}_{j}^{\dagger}$ denote the irreducible component of $\bar{Y}^{\dagger}$ which intersects $\bar{Y}_{0}$ in $\eta_{j}$. Write $\eta_{j}^{\dagger} \in \bar{Y}_{j}^{\dagger}$ for the point where $\bar{Y}_{j}^{\dagger}$ intersects $\bar{Y}_{0}$. Let $G_{j}^{\dagger} \subset G^{\dagger}$ be the subgroup generated by $\boldsymbol{\mu}_{p}(\bar{K})$ and $H_{j}$. One checks that $\left(\bar{f}_{j}^{\dagger}: \bar{Y}_{j}^{\dagger} \rightarrow \bar{X}_{j}^{\dagger}, \eta_{j}^{\dagger}, H_{j}\right)$ is a pointed $G_{j}^{\dagger}$-tail cover with ramification type $\left(m_{j}, h_{j}\right)$, totally ramified above $\tau_{j}$. See $\$ 2.2 .2$

Let $\operatorname{Aut}_{k}^{0}\left(\bar{Y}^{\dagger}\right)$ denote the group of $k$-linear automorphisms of $\bar{Y}^{\dagger}$ which normalize the action of $G^{\dagger}$, commute with the action of $H_{0} \subset G^{\dagger}$ and restrict to the identity on $\bar{Y}_{0}$. We have seen in $\$ 3.3 .3$ that the absolute monodromy action on $\bar{f}^{\dagger}$ is a homomorphism

$$
\bar{\kappa}^{\dagger}: \operatorname{Gal}\left(\bar{K} / K_{0}\right) \longrightarrow \operatorname{Aut}_{k}^{0}\left(\bar{Y}^{\dagger}\right) .
$$

Similarly, let $\operatorname{Aut}_{k}^{0}\left(\bar{Y}_{j}^{\dagger}\right)$ denote the group of $k$-linear automorphisms of $\bar{Y}_{j}^{\dagger}$ which normalize the action of $G_{j}^{\dagger}$, commute with the action of $H_{j} \subset G_{j}^{\dagger}$ and fix the point $\eta_{j}$. By Proposition 3.10 , the group $\operatorname{Aut}_{k}^{0}\left(\bar{Y}_{j}^{\dagger}\right)$ is cyclic of order $(p-1) h_{j}$; 
furthermore, restricting $\bar{\kappa}^{\dagger}$ to $\bar{Y}_{j}^{\dagger}$ yields a surjective homomorphism

$$
\bar{\kappa}_{j}^{\dagger}: \operatorname{Gal}\left(\bar{K} / K_{0}\right) \longrightarrow \operatorname{Aut}_{k}^{0}\left(\bar{Y}_{j}^{\dagger}\right) \cong \mathbb{Z} /(p-1) h_{j} .
$$

4.2.2. Fix $j \notin B_{\text {wild }}$. We denote by $\bar{X}_{j, \bar{\infty}}$ (resp. $\left.\bar{X}_{j, \bar{\infty}}^{\dagger}\right)$ the generic point of the completion of the tail $\bar{X}_{j}$ (resp. the tail $\bar{X}_{j}^{\dagger}$ ) at the point $\tau_{j}$. We set $\bar{Y}_{j, \bar{\infty}}:=$ $\bar{Y}_{j} \times_{\bar{X}_{j}} \bar{X}_{j, \bar{\infty}}$ and $\bar{Y}_{j, \bar{\infty}}^{\dagger}:=\bar{Y}_{j}^{\dagger} \times_{\bar{X}_{j}^{\dagger}} \bar{X}_{j, \bar{\infty}}^{\dagger}$. Let $P_{j}(\bar{f})$ be the set of pairs $\left(\lambda, \varphi_{\bar{\infty}}\right)$ consisting of the following objects:

- $\lambda: G^{\dagger} \stackrel{\sim}{\rightarrow} G_{0}$ is an isomorphism which induces the identity on $H_{0}$, and

- $\varphi_{\bar{\infty}}: \operatorname{Ind}_{G_{j}}^{G}\left(\bar{Y}_{j, \bar{\infty}}\right) \stackrel{\sim}{\rightarrow} \operatorname{Ind}_{G_{j}^{\dagger}, \lambda}^{G}\left(\bar{Y}_{j, \bar{\infty}}^{\dagger}\right)$ is a $G$-equivariant isomorphism such that the following hold:

(1) $\varphi_{\bar{\infty}}\left(\eta_{j}\right)=\eta_{j}^{\dagger}$.

(2) The induced isomorphism $\psi_{\bar{\infty}}: \bar{X}_{j, \bar{\infty}} \stackrel{\sim}{\rightarrow} \bar{X}_{j, \bar{\infty}}^{\dagger}$ extends to an isomor$\operatorname{phism} \psi: \bar{X}_{j} \stackrel{\sim}{\rightarrow} \bar{X}_{j}^{\dagger}$.

(3) If $j \in B_{\text {prim }}$, then $\psi\left(\bar{x}_{j}\right)=\bar{x}_{j}^{\dagger}$.

(The notation ' $\operatorname{Ind}_{G_{j}^{\dagger}, \lambda}^{G}$ ' means that we embed $G_{j}^{\dagger}$ into $G$ via $\lambda: G^{\dagger} \stackrel{\sim}{\rightarrow} G_{0} \subset$ G.)

The group $\operatorname{Aut}_{k}^{0}\left(\bar{Y}_{j}^{\dagger}\right)$ acts on the set $P_{j}(\bar{f})$ from the left, as follows. Let $\left(\lambda, \varphi_{\bar{\infty}}\right)$ be an element of $P_{j}(\bar{f})$ and $\gamma$ an element of $\operatorname{Aut}_{k}^{0}\left(\bar{Y}_{j}^{\dagger}\right)$. Let $\operatorname{inn}\left(\gamma^{-1}\right): G^{\dagger} \stackrel{\sim}{\rightarrow} G^{\dagger}$ denote the unique automorphism of $G^{\dagger}$ which sends an element $\alpha \in I^{\dagger}$ to $\gamma^{-1} \circ \alpha \circ \gamma$ and induces the identity on $H_{0} \subset G^{\dagger}$. Set $\lambda^{\prime}:=\lambda \circ \operatorname{inn}\left(\gamma^{-1}\right)$. One checks that $\gamma$ extends uniquely to a $G$-equivariant isomorphism

$$
\tilde{\gamma}: \operatorname{Ind}_{G_{j}^{\dagger}, \lambda}^{G}\left(\bar{Y}_{j}^{\dagger}\right) \stackrel{\sim}{\longrightarrow} \operatorname{Ind}_{G_{j}^{\dagger}, \lambda^{\prime}}^{G}\left(\bar{Y}_{j}^{\dagger}\right) .
$$

Let $\varphi_{\bar{\infty}}^{\prime}$ be the composition of $\varphi_{\bar{\infty}}$ with the local isomorphism induced by $\tilde{\gamma}$. Then $\left(\lambda^{\prime}, \varphi_{\bar{\infty}}^{\prime}\right)$ is an element of $P_{j}(\bar{f})$. This defines the action of $\operatorname{Aut}_{j}^{0}\left(\bar{Y}_{j}^{\dagger}\right)$ on $P_{j}(\bar{f})$. It is easy to see that this action makes $P_{j}(\bar{f})$ a principal homogeneous $\operatorname{Aut}_{k}^{0}\left(\bar{Y}_{j}^{\dagger}\right)$-space. Via the monodromy action $\bar{\kappa}_{j}^{\dagger}$, we obtain a transitive Galois action of $\operatorname{Gal}\left(\bar{K} / K_{0}\right)$ on $P_{j}(\bar{f})$.

Define

$$
P(\bar{f}):=\left\{\left(\lambda ; \varphi_{j, \bar{\infty}}\right) \mid\left(\lambda, \varphi_{j, \bar{\infty}}\right) \in P_{j}(\bar{f}) \forall j\right\} .
$$

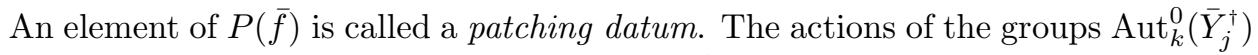
on $P_{j}(\bar{f})$ extend uniquely to an action of $\operatorname{Aut}_{k}^{0}\left(\bar{Y}^{\dagger}\right)$ on $P(\bar{f})$ (use Proposition 3.10 $(1))$. Again, this action is transitive and fixed point free. We consider $P(\bar{f})$ as a $\operatorname{Gal}\left(\bar{K} / K_{0}\right)$-set, via the monodromy action $\bar{\kappa}^{\dagger}$.

Proposition 4.4. The set $P(\bar{f})$ of patching data has exactly $(p-1) \cdot \prod_{j} h_{j}$ elements. The $\operatorname{Gal}\left(\bar{K} / K_{0}\right)$-orbits have length

$$
N:=(p-1) \cdot \operatorname{lcm}_{j}\left(h_{j}\right) .
$$

In particular, the action of $\operatorname{Gal}\left(\bar{K} / K_{0}\right)$ on $P(\bar{f})$ is tamely ramified.

Proof. By Proposition 3.10 and the freeness of the action of $\operatorname{Aut}_{k}^{0}\left(\bar{Y}^{\dagger}\right)$ on $P(\bar{f})$, it suffices to show that $P(\bar{f})$ is nonempty. But this follows from Lemma2.12, 
4.2.3. We shall now define a $\operatorname{Gal}\left(\bar{K} / K_{0}\right)$-equivariant map

$$
L(\bar{f}) \longrightarrow P(\bar{f}),
$$

as follows. Let $f_{R}: Y_{R} \rightarrow X_{R}$ be a lift of $\bar{f}$. Let $f^{\text {aux }}: Y^{\text {aux }} \rightarrow X$ be the auxiliary cover associated to $f$ and the set $\left(x_{j}\right)_{j \in B}$. Let $f_{R}^{\text {aux }}: Y_{R}^{\text {aux }} \rightarrow X_{R}$ denote the stable model of the auxiliary cover $f^{\text {aux }}$. By Proposition 4.3 (1), there exists a $K$-linear isomorphism $\varphi_{K}: Y_{K}^{\text {aux }} \stackrel{\sim}{\rightarrow} Y_{K}^{\dagger}$ which induces the identity on $X_{K}$. By the uniqueness of the stable model, $\varphi_{K}$ extends to an isomorphism $\varphi_{R}: Y_{R}^{\text {aux }} \stackrel{\sim}{\rightarrow} Y_{R}^{\dagger}$. Let $\bar{\varphi}: \bar{Y}^{\text {aux }} \stackrel{\sim}{\rightarrow} \bar{Y}^{\dagger}$ denote the restriction of $\varphi_{R}$ to the special fiber. After composing $\varphi_{K}$ with an element of $G^{\dagger}$, we may assume that $\varphi_{K}$ commutes with the action of $H_{0}$ (which is a subgroup of $G_{0}$ and $G^{\dagger}$ ) and that $\bar{\varphi}$ induces the identity on $\bar{Y}_{0}$ (which is a component of $\bar{Y}^{\text {aux }}$ and $\bar{Y}^{\dagger}$ ). Note that this determines $\varphi_{K}$ uniquely. Let $\lambda: G_{0} \stackrel{\sim}{\rightarrow} G^{\dagger}$ be the isomorphism $\alpha \mapsto \varphi_{K} \circ \alpha \circ \varphi_{K}^{-1}$. Fix $j \notin B_{\text {wild }}$. Let $\bar{Y}_{j}^{\text {aux }}$ denote the component of $\bar{Y}^{\text {aux }}$ which intersects $\bar{Y}_{0}$ in $\eta_{j}$, and set $\bar{Y}_{j, \infty}^{\text {aux }}:=\bar{Y}_{j}^{\text {aux }} \times_{\bar{X}_{j}} \bar{X}_{j, \bar{\infty}}$. The isomorphism (23) induces a $G$-equivariant isomorphism

$$
\operatorname{Ind}_{G_{j}}^{G}\left(\bar{Y}_{j, \bar{\infty}}\right) \stackrel{\sim}{\longrightarrow} \operatorname{Ind}_{G_{0}}^{G}\left(\bar{Y}_{j, \infty}^{\text {aux }}\right) .
$$

Let $\varphi_{j, \bar{\infty}}: \operatorname{Ind}_{G_{j}}^{G}\left(\bar{Y}_{j, \bar{\infty}}\right) \stackrel{\sim}{\rightarrow} \operatorname{Ind}_{G^{\dagger}}^{G}\left(\bar{Y}_{j, \bar{\infty}}^{\dagger}\right)$ be the composition of this isomorphism with the isomorphism induced by $\bar{\varphi}$. By construction, the tuple $\left(\lambda ; \varphi_{j, \bar{\infty}}\right)$ is a patching datum. This defines the map (24).

We claim that (24) is $\operatorname{Gal}\left(\bar{K} / K_{0}\right)$-equivariant. Indeed, for any $\sigma \in \operatorname{Gal}\left(\bar{K} / K_{0}\right)$, we may identify the auxiliary cover of the twisted cover ${ }^{\sigma} f_{R}:=f_{R} \otimes_{R}^{\sigma} R$ with the $\sigma$-twist ${ }^{\sigma}\left(f_{R}^{\text {aux }}\right):=f_{R}^{\text {aux }} \otimes_{R}^{\sigma} R$ of the auxiliary cover of $f_{R}$. Let $\kappa_{R, \sigma}^{\dagger}:{ }^{\sigma}\left(Y_{R}^{\dagger}\right) \stackrel{\sim}{\rightarrow} Y_{R}^{\dagger}$ be the unique extension of the canonical $K$-linear isomorphism ${ }^{\sigma} Y_{K}^{\dagger} \stackrel{\sim}{\rightarrow} Y_{K}^{\dagger}$ induced from the $K_{0}$-model $Y_{K_{0}}^{\dagger}$. The restriction of $\kappa_{R, \sigma}$ to the special fiber is equal to $\bar{\kappa}^{\dagger}(\sigma)$, i.e. the monodromy action of $\sigma$ on $\bar{Y}^{\dagger}$. The composition $\varphi_{R}^{\prime}:=\kappa_{R, \sigma}^{\dagger} \circ \varphi_{R}$ is the unique isomorphism which induces an $H_{0}$-equivariant isomorphism of mere covers $f_{K}^{\text {aux }} \cong f_{K}^{\dagger}$ and whose restriction to the special fiber is the identity on $\bar{Y}_{0}$. It follows that the map (24) maps the class of the twisted lift ${ }^{\sigma} f_{R}$ to the image of $\left(\lambda ; \varphi_{j, \bar{\infty}}\right)$ under the action of $\bar{\kappa}^{\dagger}(\sigma) \in \operatorname{Aut}_{k}^{0}\left(\bar{Y}^{\dagger}\right)$. This proves the claim that (24) is $\operatorname{Gal}\left(\bar{K} / K_{0}\right)$-equivariant.

Theorem 4.5. The map (24) is an isomorphism of $\operatorname{Gal}\left(\bar{K} / K_{0}\right)$-sets.

Proof. We will show that (24) is an isomorphism by constructing an inverse $P(\bar{f}) \rightarrow$ $L(\bar{f})$. Let $\left(\lambda ; \bar{\varphi}_{j, \bar{\infty}}\right) \in P(\bar{f})$ be a patching datum. We have to construct a lift $f_{R}: Y_{R} \rightarrow X_{R}$ of $\bar{f}$ which is mapped to $\left(\lambda ; \bar{\varphi}_{j, \bar{\infty}}\right)$ by (24).

By definition, the local isomorphism $\bar{\psi}_{j, \bar{\infty}}: \bar{X}_{j, \bar{\infty}} \stackrel{\sim}{\rightarrow} \bar{X}_{j, \bar{\infty}}^{\dagger}$ extends to a global isomorphism $\bar{\psi}_{j}: \bar{X}_{j} \stackrel{\sim}{\rightarrow} \bar{X}_{j}^{\dagger}$. Moreover, if $j \in B_{\text {prim }}$, then $\psi_{j}\left(\bar{x}_{j}\right)=\bar{x}_{j}^{\dagger}$. Let $\bar{\psi}: \bar{X} \stackrel{\sim}{\rightarrow} \bar{X}^{\dagger}$ denote the isomorphism which is equal to the identity on the original component and which is equal to $\bar{\psi}_{j}$ on the tails. We define $X_{R}:=X_{R}^{\dagger}$ as a marked curve over $R$. However, we identify the special fiber of $X_{R}$ with $\bar{X}$ via the isomorphism $\bar{\psi}$.

Let $\hat{X}_{R}$ denote the formal completion of $X_{R}$ along $\bar{X}$. For $j \notin B_{\text {wild }}$, let $\mathcal{D}_{j} \subset \hat{X}_{R}$ be the open formal subscheme corresponding to $\bar{X}_{j}-\left\{\tau_{j}\right\} \subset \bar{X}$ and let $\mathcal{X}_{j}$ be the completion of $X_{R}$ at $\tau_{j}$. Let $\mathcal{U}^{\circ} \subset \hat{X}_{R}$ be the open formal subscheme corresponding to $\bar{X}_{0}-\left\{\tau_{j} \mid j \in B_{\text {prim }} \cup B_{\text {new }}\right\} \subset \bar{X}$. Let $\mathcal{U}$ denote the disjoint union of $\mathcal{U}^{\circ}$ with all 
the $\mathcal{X}_{j}$. The fiber product

$$
\mathcal{B}_{j}:=\mathcal{U} \times_{\hat{X}_{R}} \mathcal{D}_{j} \cong \operatorname{Spec} R[[T]]\left\{T^{-1}\right\}
$$

is the 'boundary' of the formal disk $\mathcal{D}_{j}$. Its special fiber $\mathcal{B}_{j} \otimes_{R} k$ is canonically isomorphic to $\bar{X}_{j, \bar{\infty}}^{\dagger}$ and isomorphic to $\bar{X}_{j, \bar{\infty}}$ via $\bar{\psi}_{j, \bar{\infty}}$. Define

$$
\mathcal{V}:=\operatorname{Ind}_{G^{\dagger}, \lambda}^{G}\left(\hat{Y}_{R}^{\dagger} \times_{\hat{X}_{R}} \mathcal{U}\right) .
$$

Furthermore, let $\mathcal{E}_{j} \rightarrow \mathcal{D}_{j}$ be the (unique) tame lift of the (not necessarily connected) $G$-cover

$$
\bar{Y} \times_{\bar{X}}\left(\bar{X}_{j}-\left\{\tau_{j}\right\}\right) \longrightarrow \bar{X}_{j}-\left\{\tau_{j}\right\} \stackrel{\bar{\psi}_{j}}{\longrightarrow} \bar{X}_{j}^{\dagger}-\left\{\tau_{j}\right\}
$$

which is unramified away from the section $x_{j, R}: \operatorname{Spec} R \rightarrow \mathcal{D}_{j}$. The local isomorphism $\varphi_{j, \bar{\infty}}$ yields a $G$-equivariant isomorphism

$$
\left(\mathcal{E}_{j} \times_{\mathcal{D}_{j}} \mathcal{B}_{j}\right) \otimes_{R} k \cong \operatorname{Ind}_{G_{j}}^{G}\left(\bar{Y}_{j, \bar{\infty}}\right) \stackrel{\bar{\varphi}_{j, \infty}}{\longrightarrow} \operatorname{Ind}_{G^{\dagger}, \lambda}^{G}\left(\bar{Y}_{j, \bar{\infty}}^{\dagger}\right) \cong\left(\mathcal{V} \times_{\mathcal{U}} \mathcal{B}_{j}\right) \otimes_{R} k
$$

of étale $\bar{X}_{j, \bar{\infty}}^{\dagger}$-schemes. It lifts canonically to a $G$-equivariant isomorphism

$$
\varphi_{j}: \mathcal{E}_{j} \times_{\mathcal{D}_{j}} \mathcal{B}_{j} \stackrel{\sim}{\longrightarrow} \mathcal{V} \times \mathcal{U} \mathcal{B}_{j}
$$

of étale formal $\mathcal{B}_{j}$-schemes. By a theorem of Ferrand-Raynaud [FR70] (see also [Pri00]), there exists a formal $\hat{X}_{R}$-scheme $\mathcal{Y}$ with $G$-action such that $\mathcal{E}_{j}=\mathcal{Y} \times_{\hat{X}_{R}} \mathcal{D}_{j}$ and $\mathcal{V}=\mathcal{Y} \times_{\hat{X}_{R}} \mathcal{U}$. By Grothendieck's existence theorem, $\mathcal{Y}$ is the formal completion of a projective $R$-curve $Y_{R}$. By construction, the natural map $f_{R}: Y_{R} \rightarrow X_{R}$ is a lift of $\bar{f}$ which is mapped by (24) to the patching datum $\left(\lambda ; \bar{\varphi}_{j, \bar{\infty}}\right)$. This completes the proof of the theorem.

4.3. Some applications of Theorem 4.5. Let $k$ be an algebraically closed field of characteristic $p>0$. We denote by $K_{0}$ the fraction field of $W(k)$ and by $\bar{K}$ an algebraic closure of $K_{0}$. Theorem 4.5 together with Proposition 4.4 implies

Corollary 4.6. Let $\bar{f}: \bar{Y} \rightarrow \bar{X}$ be a special $G$-map defined over $k$. Then there exists a three point $G$-cover $f: Y \rightarrow X:=\mathbb{P}_{\bar{K}}^{1}$ whose stable reduction is isomorphic to $\bar{f}$.

This corollary is used in BW02 to determine the stable reduction of all three point covers with Galois group $\operatorname{PSL}_{2}(p)$. In particular, this gives a new proof for the result of Deligne and Rapoport on the reduction of the modular curves $X_{0}(p)$ and $X_{1}(p)$.

In addition to the existence of three point covers with given stable reduction, Theorem 4.5 and Proposition 4.4 yield interesting arithmetic information about a given three point cover.

Corollary 4.7. Let $f: Y \rightarrow X=\mathbb{P}_{\bar{K}}^{1}$ be a three point Galois cover with mild reduction. Let $\left(m_{j}, h_{j}\right)_{j \in B}$ be the signature of the stable reduction of $f$. The cover $f$ has stable reduction over the unique tame extension $K^{\text {st }} / K_{0}$ of degree

$$
N:=(p-1) \cdot \operatorname{lcm}_{j \notin B_{\text {wild }}}\left(h_{j}\right) .
$$

In particular, any three point Galois cover $f: Y \rightarrow X$ whose degree is not divisible by $p^{2}$ can be defined over a tame extension of $K_{0}$. 
Proof. Let $f_{R}: Y_{R} \rightarrow X_{R}$ be a stable model of $f$, and $\bar{f}: \bar{Y} \rightarrow \bar{X}$ the stable reduction. If $\bar{f}$ is not exceptional, then the corollary follows from Proposition 3.10 and Theorem 4.5. We may therefore assume that $\bar{f}$ is exceptional. We will also assume that we are in case (2) of Definition 2.1 leaving case (1) for the reader. By Proposition 2.2, the curve $\bar{X}$ consists of the original component and a single new tail $\bar{X}_{j_{0}}$.

Let $f_{R}^{\text {aux }}: Y_{R}^{\text {aux }} \rightarrow X_{R}$ denote the auxiliary cover associated to $f_{R}$, see 4.1 .3 . The Galois group of $f_{R}^{\text {aux }}$ is a subgroup $G_{0} \subset G$, which is a direct product $G_{0}=I_{0} \times H_{0}$. Here $I_{0} \cong \mathbb{Z} / p$ is the inertia group of a component $\bar{Y}_{0}$ of $\bar{Y}$ over $\bar{X}_{0}$ and $H_{0}$ is the Galois group of the cover $\bar{Z}_{0}=\bar{Y}_{0}^{(p)} \rightarrow \bar{X}_{0}$. Let $W_{R}:=Y_{R}^{\text {aux }} / H_{0}$. Then $W_{R} \rightarrow X_{R}$ is a stable model of the $p$-cyclic Galois cover $W_{K} \rightarrow X_{K}=\mathbb{P}_{K}^{1}$, which is ramified at 0,1 and $\infty$. Therefore, the curve $W_{K}$ has an equation of the form

$$
y^{p}=f(x):=c x^{a}(x-1)^{b},
$$

with integers $a, b$ such that $a, b, a+b \not \equiv 0 \bmod p$ and a unit $c \in R^{\times}$. After enlarging the field $K$ if necessary and a change of coordinates we may assume that $c \in W(k)^{\times}$ and $f(a /(a+b)) \in\left(W(k)^{\times}\right)^{p}$. It is known from CM88, (see also Leh01]) that the model $X_{R}$ which leads to the stable reduction of $W_{K} \rightarrow X_{K}$ is the blowup of $\mathbb{P}_{R}^{1}$ in the disk

$$
D:=\left\{x \mid \operatorname{val}\left(x-\frac{a}{a+b}\right) \geq \frac{p \operatorname{val} p}{2(p-1)}\right\} .
$$

Moreover, the stable model $W_{R} \rightarrow X_{R}$ descends to a stable model over the ring

$$
R^{\prime}:=W(k)\left[p^{1 / 2(p-1)}, f\left(\frac{a}{a+b}\right)^{1 / p}\right]=W(k)\left[p^{1 / 2(p-1)}\right] .
$$

Note that the (admissible) $H_{0}$-cover $\bar{Y}^{\text {aux }} \rightarrow \bar{W}:=W_{R} \otimes_{R} k$ is ramified at the unique singular point of $\bar{W}$ of order $m=h / 2$. Therefore, the stable model $f_{R}^{\text {aux }}$ : $Y_{R}^{\text {aux }} \rightarrow X_{R}$ descends to a stable model over the ring $R^{\prime \prime}:=W(k)\left[p^{1 / h(p-1)}\right]$. Using the method of the proof of Theorem [4.5, it is not hard to show that $f_{R}: Y_{R} \rightarrow X_{R}$ descends to a stable model over $R^{\prime \prime}$. This finishes the proof of the corollary.

The last corollary can be refined in order to obtain a formula for the ramification index of the field of moduli of a three point cover in terms of its stable reduction.

Corollary 4.8. Let $\bar{f}: \bar{Y} \rightarrow \bar{X}$ be a special $G$-map, with signature $\left(m_{j}, h_{j}\right)_{j \in B}$. Let $P \subset G$ denote a $p$-Sylow of $G$ and let $n:=\left[N_{G}(P): C_{G}(P)\right]$ be the index of the centralizer of $P$ inside the normalizer of $P$. Recall that $\tilde{L}(\bar{f})$ denotes the set of isomorphism classes of three point $G$-covers defined over $\bar{K}$ whose stable reduction is isomorphic to $\bar{f}$. There exists a positive integer $n^{\prime}$, dividing $n$ and depending only on $\bar{f}$, such that

$$
|\tilde{L}(\bar{f})|=\frac{p-1}{n^{\prime}} \prod_{j \notin B_{\text {wild }}} \frac{h_{j}}{\left|\operatorname{Aut}_{G}\left(\bar{f}_{j}\right)\right|} .
$$

Let $f: Y \rightarrow X$ be a lift of $\bar{f}$. Then the field of moduli $K^{\text {in }}$ of $f$ (relative to the extension $\left.\bar{K} / K_{0}\right)$ is the unique tame extension of $K_{0}$ of degree

$$
N^{\prime}:=\frac{p-1}{n^{\prime}} \operatorname{lcm}_{j \notin B_{\text {wild }}} \frac{h_{j}}{\left|\operatorname{Aut}_{G}\left(\bar{f}_{j}\right)\right|} .
$$


Proof. We have seen in $\$ 4.1 .2$ that $\tilde{L}(\bar{f})=L(\bar{f}) / \operatorname{Aut}_{G}^{0}(\bar{f})$. Moreover, the stabilizer in $\operatorname{Aut}_{G}^{0}(\bar{f})$ of any element of $L(\bar{f})$ is equal to $C_{G}$. Let $n^{\prime}$ be the order of the image of the homomorphism $\operatorname{Aut}_{G}^{0}(\bar{f}) \rightarrow G / C_{G}$ which is defined by Lemma 2.17 It is easy to see that $n^{\prime}$ divides $n$. We see that Corollary 4.8 follows from Proposition 3.10, Theorem 4.5 and Lemma 2.17.

4.4. Genus zero dessins of degree $p$. Let $p$ be a prime and $G \subset S_{p}$ a primitive transitive permutation group of degree $p$. We write $N \subset G$ for the stabilizer of 1 . Let $f: Y \rightarrow X:=\mathbb{P}_{\overline{\mathbb{Q}}}^{1}$ be a three point Galois cover, with Galois group $G$. Then $g: Z:=Y / N \rightarrow X$ is a three point cover of degree $p$, and $f: Y \rightarrow X$ is the Galois closure of $g$. We make the following assumptions.

Assumption 4.9. (1) The subgroup $N \subset G$ is self-normalizing and $G$ is center-free.

(2) The curve $Z$ has genus 0 .

The first assumption is made only for simplicity. It ensures that the $G$-cover $f: Y \rightarrow X$ and the (non-Galois) cover $g: Z \rightarrow X$ have the same field of moduli, and that this field of moduli is also the minimal field of definition for both covers, see, e.g., [DD97. The second assumption is more restrictive. As we will see in a moment, the stable reduction of genus zero dessins is rather simple

Let $K$ be the field of moduli of $f: Y \rightarrow X$, relative to the extension $\overline{\mathbb{Q}} / \mathbb{Q}$. Let $\mathfrak{p}$ be a prime ideal of $K$ dividing $p$. There exist a finite extension $K^{\prime} / K$ and a prime $\mathfrak{p}^{\prime}$ of $K^{\prime}$ over $\mathfrak{p}$ such that $f$ has a $K^{\prime}$-model $f_{K^{\prime}}: Y_{K^{\prime}} \rightarrow X_{K^{\prime}}$ which extends to a stable model over the local ring $\mathcal{O}_{K^{\prime}, \mathfrak{p}^{\prime}}$. Let $\bar{f}: \bar{Y} \rightarrow \bar{X}$ be the stable reduction of $f_{K}^{\prime}$ at $\mathfrak{p}^{\prime}$. One checks that $\bar{f}: \bar{Y} \rightarrow \bar{X}$ depends only on the field $K$ and the prime $\mathfrak{p}$. Hence we may call $\bar{f}: \bar{Y} \rightarrow \bar{X}$ the stable reduction of $f: Y \rightarrow X$ at $\mathfrak{p}$.

Let us assume that $f: Y \rightarrow X$ has bad reduction at $\mathfrak{p}$. Note that $p$ strictly divides the order of $G$. Therefore, by Theorem 2.6 the stable reduction $\bar{f}: \bar{Y} \rightarrow \bar{X}$ is a special $G$-map, associated to a special $G$-deformation datum $\left(\bar{Z}_{0}, \omega_{0} ; \bar{Y}_{j}\right)$, see $\$ 2.2 .3$ In the following, we use freely the notation introduced in $\$ 2.2 .3$

Lemma 4.10. (1) The character $\chi: H_{0} \rightarrow \mathbb{F}_{p}^{\times}$is injective. In particular, $H_{0} \cong \mathbb{Z} / m$ with $m \mid(p-1)$.

(2) There are no new tails.

(3) For $j \in B_{\text {prim }}$, let $x_{j} \in\{0,1, \infty\}$ denote the branch point of $f: Y \rightarrow X$ which specializes to the primitive tail $\bar{X}_{j}$. The ramification invariant of the primitive tail cover $\bar{f}_{j}: \bar{Y}_{j} \rightarrow \bar{X}_{j}$ is given by the formula

$$
\sigma_{j}=\frac{\left|g^{-1}\left(x_{j}\right)\right|-1}{p-1} .
$$

Proof. Part (1) is an immediate consequence of the fact that the $p$-cyclic subgroup $I_{0}$ of $S_{p}$ is self-centralizing.

To prove (2), suppose that $f_{j}: \bar{Y}_{j} \rightarrow \bar{X}_{j}$ is a new tail, with Galois group $G_{j}$. Let $N_{j}:=N \cap G_{j}$ and $\bar{Z}_{j}:=\bar{Y}_{j} / N_{j}$. It follows from Assumption 4.9 (2) that the curve $\bar{Z}_{j}$ has genus 0 . Since $N$ has index $p$ in $G$ and $G_{j}$ contains a $p$-Sylow of $G$, the index of $N_{j}$ in $G_{j}$ is also $p$. We see that $\bar{g}_{j}: \bar{Z}_{j} \rightarrow \bar{X}_{j}$ is a cover of degree $p$ between curves of genus 0 , totally branched at $\bar{\infty}_{j} \in \bar{X}_{j}$ and étale elsewhere. This means that in suitable coordinates $\bar{g}_{j}$ is given by a monic polynomial $q \in k[z]$ of degree $p$ such that the derivative $q^{\prime}$ has degree 0 . Hence $q=z^{p}+a z+b$, and after a further normalization we get $q=z^{p}-z$. It follows that the tail cover $\bar{f}_{j}: \bar{Y}_{j} \rightarrow \bar{X}_{j}$ is an 
Artin-Schreier cover with conductor 1, i.e. $\sigma_{j}=1$. This contradicts the results of \$2. We conclude that $\bar{f}: \bar{Y} \rightarrow \bar{X}$ has no new tails, proving (2).

For $j \in B_{\text {prim }}$, let $\bar{g}_{j}: \bar{Z}_{j} \rightarrow \bar{X}_{j}$ be as before. The same argument as before shows that $\bar{g}_{j}$ is a cover between smooth curves of genus 0 of degree $p$, totally ramified at $\bar{\infty}_{j} \in \bar{X}_{j}$. But now $\bar{g}_{j}$ is tamely ramified at $\bar{x}_{j}$ and étale over $\bar{X}_{j}-\left\{\bar{\infty}_{j}, \bar{x}_{j}\right\}$ (recall that $\bar{x}_{j} \in \bar{X}_{j}$ denotes the specialization of the branch point $x_{j} \in\{0,1, \infty\}$ ). Since the stable model of $f$ is a tame cover in a neighborhood of $\bar{X}_{j}-\left\{\bar{\infty}_{j}\right\}$, we have $\left|\bar{g}_{j}^{-1}\left(\bar{x}_{j}\right)\right|=\left|g^{-1}\left(x_{j}\right)\right|$. Now (3) follows from a calculation with the RiemannHurwitz formula and the fact that $g\left(\bar{Z}_{j}\right)=0$. See, e.g., [BP], Lemma 2.3.1.

The lemma shows that the signature of $\bar{f}: \bar{Y} \rightarrow \bar{X}$ is a triple $\left(\sigma_{1}, \sigma_{2}, \sigma_{3}\right)$ of rational numbers, with $\sigma_{j}=h_{j} / m_{j}, 0 \leq h_{j}<m_{j}$ and $\left(h_{j}, m_{j}\right)=1$. It satisfies the vanishing cycle formula

$$
\sigma_{1}+\sigma_{2}+\sigma_{3}=1
$$

The special deformation datum $\left(\bar{Z}_{0}, \omega_{0}\right)$ is essentially determined by the signature. Indeed, the curve $\bar{Z}_{0}$ is isomorphic to the smooth projective model of the plane curve with the equation

$$
z^{m}=x^{h_{1}}(x-1)^{h_{2}},
$$

where $m$ is the least common multiple of the $m_{j}$, and the differential $\omega_{0}$ has the form

$$
\omega_{0}=\epsilon \frac{z \mathrm{~d} x}{x(x-1)},
$$

for some $\epsilon \in \mathbb{F}_{p}^{\times}$. Conversely, given a triple $\left(\sigma_{1}, \sigma_{2}, \sigma_{3}\right)$ satisfying all of the above conditions, one checks that (27) and (28) define a special deformation datum $\left(\bar{Z}_{0}, \omega_{0}\right)$. Therefore, the existence of a special deformation datum with a given signature imposes no restriction on the stable reduction $\bar{f}: \bar{Y} \rightarrow \bar{X}$.

Example 4.11. Let $p=7$ and $G:=S_{7}$. Suppose furthermore that the branch cycle description of the cover $g: Z \rightarrow X$ is $(6,6,2-2)$ (each entry designates a conjugacy class of $S_{7}$, e.g. '2-2' denotes the product of two 2-cycles). From the table computed by Malle Mal94 we see that there are exactly 4 non-isomorphic three point covers with monodromy group $S_{7}$ and this branch cycle description. Moreover, the absolute Galois group $\operatorname{Gal}(\overline{\mathbb{Q}} / \mathbb{Q})$ permutes these four covers transitively, with permutation group $S_{4}$. Therefore, the field of moduli of the $G$-cover $f: Y \rightarrow X$ is a degree 4 extension $K / \mathbb{Q}$.

Let $\mathfrak{p}$ be a prime ideal of $K$ over 7. Suppose that $f: Y \rightarrow X$ has bad reduction at $\mathfrak{p}$ and let $\bar{f}: \bar{Y} \rightarrow \bar{X}$ denote the stable reduction. It follows from Lemma 4.10 (3) that the signature of $\bar{f}$ is equal to $(1 / 6,1 / 6,2 / 3)$. For $j=1,2$, the RiemannHurwitz formula implies that the Galois group $G_{j}$ of the primitive tail cover $\bar{f}_{j}$ : $\bar{Y}_{j} \rightarrow \bar{X}_{j}$ is isomorphic to the semi-direct product $7: 6$. It follows that $\bar{Y}_{j} \rightarrow \bar{X}_{j}$ is given by the two equations

$$
z^{6}=x, \quad y^{7}-y=z .
$$

The third tail cover $\bar{f}_{3}: \bar{Y}_{3} \rightarrow \bar{X}_{3}$ has inertia invariant $\sigma_{3}=2 / 3$ and branch cycle description 2-2 at the tame branch point. We say that $\bar{f}_{3}$ is a primitive tail cover of type (2/3,2-2). The Galois group $G_{3}$ of $\bar{f}_{3}$ is a subgroup of $A_{7}$ containing a semi-direct product $7: 3$ and a permutation of type 2-2. By elementary group theory, $G_{3}$ is isomorphic to $A_{7}$ or to $L_{2}(7):=\mathrm{PSL}_{2}(7)$. 
It is a priori not clear how many non-isomorphic primitive tail covers of type $(2 / 3,2-2)$ exist, and what their Galois groups are. We claim that there exists in fact a unique primitive tail cover of type $(2 / 3,2-2)$, with Galois group $L_{2}(7)$. The existence of one such cover is proved in BW02. (It is obtained by reducing a certain three point cover with Galois group $L_{2}(7)$.) To prove uniqueness, we use our lifting result, Theorem 4.5

Given $\bar{f}_{3}: \bar{Y}_{3} \rightarrow \bar{X}_{3}$ as above, one shows that there exists, up to isomorphism, a unique special $G$-map $\bar{f}: \bar{Y} \rightarrow \bar{X}$ with signature $(1 / 6,1 / 6,2 / 3)$ whose third tail cover is isomorphic to $\bar{f}_{3}: \bar{Y}_{3} \rightarrow \bar{X}_{3}$. By Corollary 4.8 there exist exactly

$$
N^{\prime}:=\frac{12}{n^{\prime} \cdot\left|\operatorname{Aut}_{G_{3}}\left(\bar{f}_{3}\right)\right|}
$$

nonisomorphic three point covers $f^{\prime}: Y^{\prime} \rightarrow X^{\prime}$ whose stable reduction (at a given prime $\tilde{\mathfrak{p}}$ of $\overline{\mathbb{Q}}$ ) is isomorphic to $\bar{f}$. Moreover, all of these covers are conjugate under the action of the inertia group of $\tilde{\mathfrak{p}}$. Using Lemma 2.17] Remark 2.18] and equation (29), one shows that $n^{\prime}=3$. Thus, $N^{\prime}$ is equal to either 2 or 4 .

As we have mentioned before, all three point covers with Galois group $S_{7}$ and branch cycle description $(6,6,2-2)$ are conjugate. Hence we may assume that $f=f^{\prime}$. We conclude that for each primitive tail cover $\bar{f}_{3}$ of type $(2 / 3,2-2)$ there exists a prime $\mathfrak{p}$ in $K$ above 7 with $e(\mathfrak{p} / 7) \in\{2,4\}$. This prime $\mathfrak{p}$ is uniquely determined by the property that the stable reduction of $f: Y \rightarrow X$ at $\mathfrak{p}$ has a primitive tail cover isomorphic to $\bar{f}_{3}$. By Malle's table, we have $7=\mathfrak{p}_{1}^{2} \mathfrak{p}_{2} \mathfrak{p}_{3}$ in $K$. Therefore, our analysis of the stable reduction implies:

- The cover $f: Y \rightarrow X$ has bad reduction at $\mathfrak{p}_{1}$ and good reduction at $\mathfrak{p}_{2}$ and $\mathfrak{p}_{3}$. The stable reduction of $f: Y \rightarrow X$ at $\mathfrak{p}_{1}$ occurs after an extension which is ramified at $\mathfrak{p}_{1}$ of order 2 .

- There exists a unique primitive tail cover of type $(2 / 3,2-2)$. Its Galois group is $L_{2}(7)$.

We remark that good reduction at $\mathfrak{p}_{2}$ and $\mathfrak{p}_{3}$ does not follow from the results of Ray99, because $S_{7}$ has only one conjugacy class of elements of order 7 .

Example 4.12. As before, let $p=7$ and $G=S_{7}$. But now suppose that the branch cycle description of the cover $f: Y \rightarrow X$ is $(2-3,2-3,7)$. By Malle's table there are 9 such covers, up to isomorphism. Note that they all have bad reduction to characteristic 7 , because of the ramification index 7 .

It follows from Lemma 4.10 (3) that the signature of $\bar{f}: \bar{Y} \rightarrow \bar{X}$ is equal to $(1 / 2,1 / 2,0)$. For $j=1,2$, the Galois group $G_{j}$ of the tail cover $\bar{f}_{j}: \bar{Y}_{j} \rightarrow \bar{X}_{j}$ contains a 7 -cycle and a 2 -cycle. Hence, by a theorem of Jordan we have $G_{j}=S_{7}$.

We claim that there exists, up to isomorphism, a unique primitive tail cover $\bar{f}_{1}: \bar{Y}_{1} \rightarrow \bar{X}_{1}$ of type $(1 / 2,2-3)$ with Galois group $S_{7}$. We have already shown its existence. To prove uniqueness, we use again Theorem 4.5]

Let us choose a point $\eta_{1} \in \bar{Y}_{1}$ above the wild branch point $\bar{\infty}_{1} \in \bar{X}_{1}$. Since $\sigma_{1}=1 / 2$, the inertia group at $\eta_{1}$ is a dihedral group $G_{0}=I_{0} \rtimes H_{0}$, with $\left|I_{0}\right|=7$ and $H_{0}=|2|$. Recall that the triple $\left(\bar{f}_{1}, \eta_{1}, H_{0}\right)$ is called a pointed $S_{7}$-tail cover. Let $\bar{f}_{2}: \bar{Y}_{2} \rightarrow \bar{X}_{2}$ be a copy of the $S_{7}$-tail cover $\bar{f}_{1}$. It is easy to see that there are exactly three points on $\bar{Y}_{2}$ above $\bar{\infty}_{2}$ with inertia group $G_{0}$. Let $\eta_{2}$ be one of them. Then $\left(\bar{f}_{2}, \eta_{2}, H_{0}\right)$ is another pointed $S_{7}$-tail cover. It is isomorphic to $\left(\bar{f}_{1}, \eta_{1}, H_{0}\right)$ (by an isomorphism commuting with the action of $S_{7}$ ) if and only if $\eta_{1}=\eta_{2}$. Finally, let $\left(\bar{Z}_{0}, \omega_{0}\right)$ be the (unique) special deformation datum with signature $(1 / 2,1 / 2,0)$. 
One checks that $\left(\bar{Z}_{0}, \omega_{0}, \bar{Y}_{1}, \bar{Y}_{2}\right)$ gives rise to a special $G$-deformation datum and hence to a special $G$-map $\bar{f}: \bar{Y} \rightarrow \bar{X}$, see $\$ 2.2 .3$ Moreover, by varying the point $\eta_{2}$ we obtain exactly three non-isomorphic special $G$-maps with signature $(1 / 2,1 / 2,0)$ and whose primitive tail covers are both isomorphic to $\bar{f}_{1}: \bar{Y}_{1} \rightarrow \bar{X}_{1}$.

Choose one of the three special $G$-maps $\bar{f}: \bar{Y} \rightarrow \bar{X}$ constructed from $\bar{f}_{1}: \bar{Y}_{1} \rightarrow$ $\bar{X}_{1}$. By Corollary 4.8 there exist exactly $N^{\prime}=6 / n^{\prime}$ non-isomorphic three point covers $f^{\prime}: Y^{\prime} \rightarrow X$ with Galois group $S_{7}$ whose stable reduction (at a given place $\tilde{\mathfrak{p}}$ above 7 ) is isomorphic to $\bar{f}$. Moreover, they form a single orbit under the action of the inertia group at $\tilde{\mathfrak{p}}$. Using Remark 2.18 we see that $2 \mid n^{\prime}$. Using the fact that $S_{7}$ has no outer automorphism, one further shows that $n^{\prime}=2$ and hence $N^{\prime}=3$. Therefore, for each primitive tail cover $\bar{f}_{1}: \bar{Y}_{1} \rightarrow \bar{X}_{1}$ we obtain, by our lifting result, exactly 9 non-isomorphic three point covers with Galois group $S_{7}$ and branch cycle description $(2-3,2-3,7)$. However, there are only 9 such covers altogether. This proves that $\bar{f}_{1}: \bar{Y}_{1} \rightarrow \bar{X}_{1}$ is indeed unique, up to isomorphism. As a by-product, we have also shown that the absolute ramification index of any prime ideal $\mathfrak{p}$ dividing 7 in the field of moduli of $f: Y \rightarrow X$ is equal to 3 .

By Mal94 the set of 9 isomorphism classes form two Galois orbits, of length 3 and 6 respectively, corresponding to extensions $K_{1} / \mathbb{Q}$ and $K_{2} / \mathbb{Q}$. In $K_{1}$ we have $7=\mathfrak{p}_{1}^{3}$ and in $K_{2}$ we have $7=\mathfrak{p}_{2}^{3} \mathfrak{p}_{3}^{3}$. This confirms our prediction.

Remark 4.13. One can use Malle's table Mal94 to verify our results in many more examples. In particular, one gets a numerical verification of Theorem 1 of the Introduction for all genus zero dessins of degree $\leq 10$. One also sees that the condition that $p$ strictly divides the order of $G$ is necessary for Theorem 1 to hold. For instance, one finds several three point covers of degree $n \geq 10$, with monodromy group $S_{n}$ or $A_{n}$, such that 5 is wildly ramified in the field of moduli.

Remark 4.14. The relation between the existence of three point covers with bad reduction on the one hand and tail covers with low ramification invariant on the other hand is exploited more systematically in $[\mathrm{BP}$. In Zap02 Zapponi studies the stable reduction of genus zero dessins of degree $p$ which are totally ramified at one point, by analyzing the defining equation. His results confirm our results on dessins of genus 0 .

\section{REFERENCES}

[Bec89] S. Beckmann, Ramified primes in the field of moduli of branched coverings of curves, J. of Algebra 125 (1989), 236-255. MR 90i:11063

[BM00] J. Bertin and A. Mézard, Déformations formelles des revêtements sauvagement ramifiés de courbes algébriques, Invent. Math. 141 (2000), 195-238. MR 2001f:14023

[BP] I.I. Bouw and R.J Pries, Rigidity, reduction, and ramification, To appear in Math. Ann.

[BW00] I.I. Bouw and S. Wewers, Reduction of covers and Hurwitz spaces, arXiv:math. AG/0005120, 2000.

[BW02] I.I. Bouw and S. Wewers, Stable reduction of modular curves, arXiv:math.AG/0210363, 2002.

[CM88] R. Coleman and W. McCallum, Stable reduction of Fermat curves and Jacobi sum Hecke characters, J. Reine Angew. Math. 385 (1988), 41-101. MR 89h:11026

[DD97] P. Dèbes and J.-C. Douai, Algebraic covers: Field of moduli versus field of definition, Ann. Scient. Éc. Norm. Sup. 30 (1997), 303-338. MR 98k:11081

[FR70] D. Ferrand and M. Raynaud, Fibres formelles d'un anneau local noethérien, Ann. Scient. Éc. Norm. Sup. 3 (1970), 295-311. MR 42:7660

[GM99] B. Green and M. Matignon, Order $p$ automorphisms of the open disc of a p-adic field, J. Amer. Math. Soc. 12 (1999), no. 1, 269-303. MR 99j:13017 
[Hen00] Y. Henrio, Arbres de Hurwitz et automorphismes d'ordre $p$ des disques et des couronnes p-adic formels, To appear in: Comp. Math., available at arXiv:math.AG/0011098, 2000.

[Knu83] F.F. Knudsen, The projectivity of the moduli space of stable curves, II, Math. Scand. 52 (1983), 161-199. MR 85d:14038a

[Koe] B. Koeck, Belyi's theorem revisited, arXiv:math.AG/0108222.

[Leh01] C. Lehr, Reduction of p-cyclic covers, Manuscripta Math. 106 (2001), 151-175. MR 2002h:14049

[Mal94] G. Malle, Fields of definition of some three point ramified field extensions, The Grothendieck Theory of Dessins d'Enfants (L. Schneps, ed.), London Math. Soc. Lecture Note Series, no. 200, Cambridge Univ. Press, 1994, pp. 147-168. MR 95m:11128

[Mil80] J.S. Milne, Étale cohomology, Princeton Univ. Press, 1980. MR 81j:14002

[Pri00] R.J. Pries, Construction of covers with formal and rigid geometry, Courbes semi-stables et groupe fondamental en géométrie algébrique (J.-B. Bost, F. Loeser, and M. Raynaud, eds.), Progress in Math., no. 187, Birkhäuser, 2000, pp. 157-167. MR 2001e:14026

[Pri02] R.J. Pries, Families of wildly ramified covers of curves, Amer. J. Math. 124 (2002), no. $4,737-768$.

[Ray74] M. Raynaud, Schémas en groupes de type $(p, \ldots, p)$, Bull. Soc. Math. Fr. 102 (1974), 241-280. MR 54:7488

[Ray90] M. Raynaud, p-groupes et réduction semi-stable des courbes, Grothendieck Festschrift III (P. Cartier, ed.), Progress in Math., no. 88, Birkhäuser, 1990, pp. 179-197. MR 92m:14025

[Ray99] M. Raynaud, Spécialisation des revêtements en caractéristique $p>0$, Ann. Scient. Éc. Norm. Sup. 32 (1999), no. 1, 87-126. MR 2000e:14016

[Saï01] M. Saïdi, Torsors under finite and flat group schemes of rank $p$ with Galois action, arXiv:math.AG/0106246, 2001.

[Ser68] J.-P. Serre, Corps locaux, Hermann, 1968. MR 50:7096

[Wew02] S. Wewers, Formal deformations of curves with group scheme action, arXiv:math.AG/0212145, 2002.

[Wew03] S. Wewers, Reduction and lifting of special metacyclic covers, Ann. Scient. Éc. Norm. Sup. 36 (2003), 113-138.

[Zap02] L. Zapponi, The arithmetic of prime degree trees, Int. Math. Res. Not. 4 (2002), 211-219. MR 2002j:11066

Mathematisches Institut, Beringstr. 1, 53115 Bonn, Germany

E-mail address: wewers@math.uni-bonn.de 